\title{
Softened gravity and the extension of the standard model up to infinite energy
}

\author{
Gian F. Giudice, ${ }^{a}$ Gino Isidori, ${ }^{b, c}$ Alberto Salvio $^{d}$ and Alessandro Strumia ${ }^{e, f}$ \\ ${ }^{a}$ CERN, Theory Division, \\ CH-1211 Geneva 23, Switzerland \\ ${ }^{b}$ Physik-Institut, Universität Zürich, \\ CH-8057, Zürich, Switzerland \\ ${ }^{c}$ INFN, Laboratori Nazionali di Frascati, \\ I-00044 Frascati, Italy \\ ${ }^{d}$ Departamento de Física Teórica, Universidad Autónoma de Madrid \\ and Instituto de Física Teórica IFT-UAM/CSIC, Cantoblanco, Madrid 28049, Spain \\ ${ }^{e}$ INFN - Sezione di Pisa e Dipartimento di Fisica dell'Università di Pisa, \\ I-56127 Pisa, Italy \\ ${ }^{f}$ National Institute of Chemical Physics and Biophysics, \\ Akadeemia tee 23, 12618 Tallinn, Estonia \\ E-mail: Gian.Giudice@cern.ch, isidori@physik.uzh.ch, \\ alberto.salvio@uam.es, astrumia@cern.ch
}

ABSTRACT: Attempts to solve naturalness by having the weak scale as the only breaking of classical scale invariance have to deal with two severe difficulties: gravity and the absence of Landau poles. We show that solutions to the first problem require premature modifications of gravity at scales no larger than $10^{11} \mathrm{GeV}$, while the second problem calls for many new particles at the weak scale. To build models that fulfill these properties, we classify 4dimensional Quantum Field Theories that satisfy Total Asymptotic Freedom (TAF): the theory holds up to infinite energy, where all coupling constants flow to zero. We develop a technique to identify such theories and determine their low-energy predictions. Since the Standard Model turns out to be asymptotically free only under the unphysical conditions $g_{1}=0, M_{t}=186 \mathrm{GeV}, M_{\tau}=0, M_{h}=163 \mathrm{GeV}$, we explore some of its weak-scale extensions that satisfy the requirements for TAF.

Keywords: Higgs Physics, Beyond Standard Model, Renormalization Group, Standard Model

ARXIV EPRINT: 1412.2769 


\section{Contents}

1 Introduction 2

2 Conditions for TAF: simple cases 5

2.1 One gauge coupling 6

2.2 One Yukawa coupling 6

2.3 One scalar quartic coupling 9

$\begin{array}{lll}2.4 & \text { Multiple gauge couplings } & 13\end{array}$

$\begin{array}{lll}2.5 & \text { Supersymmetric case } & 15\end{array}$

3 Conditions for TAF: general case $\quad \mathbf{1 5}$

$\begin{array}{ll}3.1 \text { Gauge couplings } & 17\end{array}$

3.2 Yukawa couplings 18

$\begin{array}{lll}3.3 & \text { Scalar quartic couplings } & 19\end{array}$

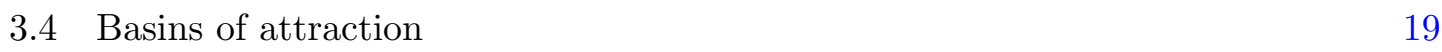

3.5 Mass parameters 20

4 Asymptotic behaviour of the Standard Model 23

$\begin{array}{lll}4.1 & \text { SM gauge couplings } & 23\end{array}$

4.2 SM Yukawa couplings 24

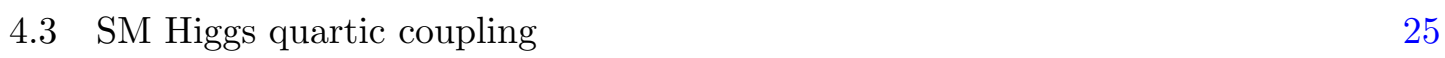

4.4 RG flow of the SM couplings 26

5 TAF extensions of the SM 28

$\begin{array}{ll}5.1 \text { Grand unification } & 28\end{array}$

5.2 TAF extensions of the SM at the weak scale 30

$\begin{array}{lll}5.3 & \text { New heavy Higgs and flavour processes } & 30\end{array}$

5.4 New heavy $\mathrm{SU}(2)_{R}$ vectors 34

6 Towards a realistic weak-scale TAF theory 36

6.1 Pati-Salam $\mathrm{SU}(2)_{L} \otimes \mathrm{SU}(2)_{R} \otimes \mathrm{SU}(4)_{\mathrm{PS}} \quad 37$

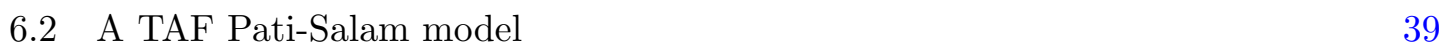

6.3 Trinification $\mathrm{SU}(3)_{L} \otimes \mathrm{SU}(3)_{R} \otimes \mathrm{SU}(3)_{c} \quad 41$

7 Conclusions $\quad 44$

A RGE for Pati-Salam models $\quad 46$

B Flavour bounds on Pati-Salam vector leptoquarks $\quad 54$

C RGE for trinification models $\quad 56$ 


\section{Introduction}

The naturalness problem of the Higgs mass is a central issue for the searches in the upcoming high-energy run of the LHC and, more generally, for defining the future strategy of particle physics. The conventional wisdom that new physics should intervene below the $\mathrm{TeV}$ and soften the quantum corrections to the Higgs mass has been seriously challenged by Run-1 LHC data. This has prompted a reconsideration of the standard approach and the study of scenarios in which the issue of naturalness is addressed without new dynamics at the weak scale affecting the quantum corrections to the Higgs mass.

A severe obstacle to this logical path is the expectation that several open questions in high-energy physics (such as quantum gravity, gauge unification, inflation, neutrino masses, baryogenesis, strong CP problem, etc.) require the existence of new heavy particles. Such particles, if sufficiently coupled to the Higgs, introduce an unavoidable naturalness problem. A radical (but admittedly questionable) approach is to ignore many of these highenergy open questions or, at least, to believe that some of these questions can be resolved by introducing only new particles that either have masses below the weak scale or are sufficiently decoupled from the Higgs [1]. If this is the case, the SM, or a mild modification of it, could be the final theory of particle physics [2-35]. However, even such a radical approach cannot ignore one problem: gravity. The unknown dynamics of quantum gravity at the Planck mass $M_{\mathrm{Pl}}$ is expected to introduce an unavoidable naturalness problem.

Our ignorance about quantum gravity leaves the door open to unexpected solutions. After all, we do not know if the Newton constant $G_{N}$ simply describes a coupling constant or signals the presence of new degrees of freedom with mass $M_{\mathrm{Pl}}$. Moreover, gravity becomes strong only at high energies and thus a UV softening of gravity could bypass the naturalness problem. In particular, if the power-law running of the gravitational interaction shuts off at a scale $\Lambda_{G}$, the gravitational corrections to the Higgs mass would amount to $\delta M_{h}^{2} \approx \ell G_{N} \Lambda_{G}^{4}$, where the coefficient $\ell$ includes couplings and loop factors. Since, in the limit in which we turn off SM interactions, the Higgs field appears in the energy-momentum tensor only with derivatives, minimally-coupled gravity respects a Higgs shift symmetry and cannot generate through quantum corrections terms in the Higgs potential at any order in perturbation theory. However, the shift symmetry is broken by SM interactions or by a direct Higgs coupling with the scalar curvature, and we expect that corrections to $M_{h}^{2}$ occur at least at two loops with $\ell \sim(4 \pi)^{-4}$. Gravity would not pose a naturalness problem as long as $\delta M_{h}^{2} \lesssim M_{h}^{2}$, which holds only if its conventional high-energy behaviour softens before reaching the scale $\Lambda_{G} \sim 4 \pi\left(M_{h} M_{\mathrm{Pl}}\right)^{1 / 2} \sim 10^{11} \mathrm{GeV}$.

In conclusion, any candidate for a theory of gravity that respects Higgs naturalness must satisfy the following properties: (i) premature UV softening of gravitational interactions below $\Lambda_{G}$; (ii) no heavy degrees of freedom with sizeable non-gravitational couplings to SM particles. We will refer to theories of this kind as softened gravity.

We do not know of any realistic working examples of softened gravity, but maybe such theories could be built out of some low-scale string theory [36] or fat-gravity [37] models. An attempt to construct a theory of softened gravity was presented in [38]. The modification of the high-energy gravitational behaviour required the presence of a spin-two 
ghost at the scale $\Lambda_{G}$ and thus the theory cannot be considered realistic. Nevertheless, this attempt teaches a useful lesson: for gravity not to introduce a hierarchy problem, general relativity must be modified at the scale $\Lambda_{G}$, i.e. well below $M_{\mathrm{Pl}}$.

Even without specifying the model of softened gravity or knowing if any such theory exists, we can nonetheless reach a general conclusion about them. Since such theories must soften the ordinary power-law energy increase starting from a scale smaller or equal to $\Lambda_{G}$, the gravitational interactions felt by SM particles remain weak at all energies, with couplings at most $\sim \Lambda_{G} / M_{\mathrm{Pl}} \sim 10^{-7}$. This means that, in the context of the hypothetical theories with softened gravity, the SM sector (or any of its extensions) is not influenced by the gravitational sector at any scale. Thus, it is sensible to investigate the behaviour of the SM using the ordinary tools of Quantum Field Theory (QFT), even at energy scales larger than $M_{\mathrm{Pl}}$. This leads us to consider another important issue.

Once we accept that a theory of softened gravity exists, we must face another problem: the scale invariance is broken at the quantum level. This breaking manifests itself in the generation of dynamical scales, such as the confinement scale in asymptotically-free gauge theories, dimensional transmutation, or Landau poles in non-asymptotically free theories. In particular, in the SM the hypercharge gauge coupling $g_{1}$ - and, consequently, all other coupling constants - hit Landau poles. The appearance of Landau poles in the Renormalisation-Group (RG) evolution corresponds to a loss of perturbative control. ${ }^{1}$ Thus, any interpretation of the dynamics lurking behind Landau poles lies completely beyond the perturbative regime and different non-perturbative physical situations can occur (see e.g. [40-44]). However, it is expected that a Landau pole signals the presence of new dynamics and that the corresponding mass threshold reintroduces a Higgs naturalness problem. In a Quantum Field Theory (QFT), the change in the short-distance behaviour associated with the Landau pole is believed to affect the mass of scalars charged under the corresponding interaction, whether new particles at that scale exist or not [46].

Landau poles of SM coupling constants are usually ignored, since they occur at energies much larger than $M_{\mathrm{Pl}}$. However, as explained above, in softened gravity the RG evolution of SM couplings is unaffected by the gravitational sector at any energy scale. As a result, the problem of Landau poles cannot be ignored and must find a solution within the sector of the SM (or one of its extensions) at the weak scale. In softened gravity, the gravitational sector cannot be of any help in preventing the appearance of SM Landau poles because of its intrinsic weakness.

So we are led to the conclusion that a theory of softened gravity satisfying Higgs naturalness must be made up of separate sectors, connected with each other only by very feeble interactions. The first is the observable sector, which contains a weak-scale extension of the SM, free from any Landau pole. Its only mass scale is of the order of the TeV. The second is a gravitational sector, which must satisfy the property of softening gravitational interactions at the scale $\Lambda_{G}$ or below. This sector could be weakly or strongly-coupled, but

\footnotetext{
${ }^{1}$ The exception found in [39], where the growth of the couplings leads to an interacting fixed point rather than to a Landau pole, is perturbatively calculable because the model has a small one-loop beta function. However this is not the case for hypercharge, which could reach an interacting fixed point only at a non-perturbative value, thereby leading to the formation of condensates at an unnaturally large scale.
} 
its interactions with the observable sector must be suppressed by the gravitational coupling, of size $\Lambda_{G} / M_{\mathrm{Pl}} \sim 10^{-7}$ or less. It has still to be proved that gravitational sectors with such properties exist. Additional sectors containing new particles (such as the inflaton, the righthanded neutrino or the axion) may exist, as long as the coupling constants $c$, describing the interaction between the new heavy particles with mass $M$ and the observable sector, are sufficiently small. If we write the contribution to the Higgs mass from heavy particles as $\delta M_{h}^{2} \approx \ell c^{2} M^{2}$, where $\ell$ counts SM couplings and loop factors, the naturalness principle (which states that $\left.\delta M_{h}^{2} \lesssim M_{h}^{2}\right)$ requires $c \lesssim 10^{-7}(4 \pi \sqrt{\ell})^{-1}\left(10^{10} \mathrm{GeV} / M\right)$. We assume that the couplings $c$ are too small to affect in any significant way the RG evolution of the coupling constants observable sector.

The goal of this paper is the construction of viable models for the observable sector. This task, for the reasons explained above, is fairly independent of the details of the gravitational sector (and of other additional sectors), as long as the hypotheses of softened gravity are satisfied. To achieve this goal, we study the conditions under which a general 4-dimensional QFT can hold up to infinite energy, with all dimensionless coupling constants (gauge couplings $g$, Yukawa couplings $y$, and scalar quartic couplings $\lambda$ ) remaining perturbative at any energy above a fixed scale $\mu_{0}$ and flowing to zero in the far UV. We will refer to this situation as Total Asymptotic Freedom (TAF).

In the first part of this paper we derive the conditions for TAF, by studying the Renormalisation Group Equations (RGE) that describe how a QFT behaves at largely different energies $\mu$. The issue had already been considered in the early Seventies [47-56], but then abandoned because of the belief that the onset of quantum gravity makes any QFT prediction above $M_{\mathrm{Pl}}$ completely irrelevant. We critically revisit the problem, highlighting the importance, for the determination of the TAF conditions, of Yukawa couplings sitting at special RG trajectories with isolated UV behaviour. The relevance of these solutions is an aspect that has often been missed in the past.

In the second part of the paper we apply our results to phenomenologically relevant theories. In particular, we address the question of constructing viable observable sectors for theories with softened gravity. Such observable sectors must be extensions of the SM that satisfy the TAF conditions, while restricting all new particles to live near the weak scale. Since these models must be based on non-abelian gauge groups, they provide an immediate explanation for the observed charge quantisation. Although we find examples of models that satisfy our criteria, we view such examples only as proofs of existence that illustrate how tough it is to build TAF theories at the weak scale. First of all, putting together the constraints from asymptotically-free couplings and from realistic flavour structures requires rather elaborate constructions with special choices of the field quantum numbers and appropriate assumptions on alignment of different flavour-violating couplings. Second, even in the most optimistic case, limits from precision and flavour-physics experiments place new particles to be well above the TeV. Such particles give physical corrections to the Higgs mass, dampening hopes for a fully natural theory.

One redeeming aspect of this unsatisfactory situation is that we have identified the most important testable prediction of softened gravity. Since any scheme of softened gravity requires the problem of SM Landau poles to be solved within the observable sector at 
the weak scale, the general prediction of such theories is that new particles must exist in the $\mathrm{TeV}$ domain. The hypercharge Landau pole requires the enlargement of the SM gauge group with new vector particles; realistic flavour structures require new fermionic particles; the Higgs embedding in the extended gauge group and the need for a correct pattern of gauge symmetry breaking require new scalar particles. The general prediction of softened gravity is the existence of many new particles around the weak scale.

This result is in open conflict with the claim, sometimes made in the literature, that the pure SM can be made natural without adding new particles at the weak scale. It also provides a way to distinguish these theories from solutions with an anthropic explanation of naturalness. Extra scalar particles at the weak scale, beyond a single Higgs boson, find no anthropic justification. The observation of "odd and unexpected" particles at the weak scale, seemingly unrelated to dynamical explanations of naturalness, but with the appropriate quantum numbers to satisfy the TAF conditions, are indicators for nonanthropic and non-dynamical solutions of naturalness, belonging to the class of theories with softened gravity. We have reached the surprising conclusion that, in spite of its vagueness, softened gravity is experimentally testable. Although it is not guaranteed that the new particles predicted by softened gravity must be within the reach of the LHC (especially because of the stringent limits from flavour physics), a possible future hadron collider in the $100-\mathrm{TeV}$ range can certain say the last word on the viability of modifications of gravity safe from the Higgs naturalness problem.

The paper is organised as follows. In section 2 we solve analytically the RGE in simple cases and derive the conditions for TAF. We also discuss how the exact solutions are related to the asymptotic behaviour valid for ultra-high energy. Building from these simple cases, in section 3 we develop a general formalism to study the high-energy asymptotic behaviour of coupling constants and determine the conditions for a theory to be TAF. In section 4 we consider, as a working example, the SM and find that it can satisfy TAF only under unphysical conditions. Motivated by naturalness, in section 5 we consider TAF extensions of the SM at the weak scale, discuss their general features, and study some of their phenomenological constraints. In section 6 we explore weak-scale TAF models based on the gauge group $\mathrm{SU}(2)_{L} \otimes \mathrm{SU}(2)_{R} \otimes \mathrm{SU}(4)_{\mathrm{PS}}$, finding a proof of existence, and models based on $\mathrm{SU}(3)_{L} \otimes \mathrm{SU}(3)_{R} \otimes \mathrm{SU}(3)_{c}$. Finally, we present our conclusions in section 7 .

\section{Conditions for TAF: simple cases}

The RGE structure is such that generally, in the perturbative regime, no coupling can flow toward non-zero UV fixed points. A possible exception, which we ignore, occurs when the one-loop $\beta$-function is accidentally small and the interplay between one- and two-loop contributions can generate a non-trivial structure. ${ }^{2}$ Since the only UV fixed points relevant for our considerations correspond to vanishing couplings, we are justified to truncate the RGE at the one-loop order.

In this section we consider simple cases where full explicit solutions to the one-loop RGE can be obtained. We define $t=\ln \left(\mu^{2} / \mu_{0}^{2}\right) /(4 \pi)^{2}$, so that $t=0$ corresponds to the

\footnotetext{
${ }^{2}$ This case was recently investigated in ref. [45].
} 
IR scale $\mu_{0}$ (which could be the weak scale or some higher reference scale) and $t \approx 0.5$ corresponds to the running from the weak to the Planck scale. Here we are interested in the behaviour for $t \rightarrow \infty$.

\subsection{One gauge coupling}

The one-loop RGE for gauge couplings is

$$
\frac{d}{d t} g^{2}=-b g^{4}
$$

where $b$ is the $\beta$-function coefficient. The solution of eq. (2.1) is

$$
\frac{1}{g^{2}}=\frac{1}{g_{0}^{2}}+b t
$$

where $g_{0}$ is the gauge coupling at the IR scale $\mu_{0}(t=0)$. For $b<0$, we encounter a Landau pole at $t_{*}=-1 /\left(g_{0}^{2} b\right)$. For $b>0$, the gauge theory is asymptotically free and the asymptotic solution is

$$
g^{2}=\frac{1}{b t} \quad \text { for } t \rightarrow \infty
$$

Thus the TAF condition is ${ }^{3}$

$$
b>0 \quad \text { (TAF condition for the gauge coupling). }
$$

TAF constrains the matter content of the theory and, in particular, excludes any Abelian gauge groups. So the SM does not satisfy TAF, because of hypercharge.

\subsection{One Yukawa coupling}

Next we consider Yukawa couplings, focusing on the case of a single (asymptotically free) gauge coupling $g$ and a single Yukawa $y$. The RGE for $y$ is

$$
\frac{d}{d t} y^{2}=y^{2}\left(f_{y} y^{2}-f_{g} g^{2}\right)
$$

where $f_{y}$ and $f_{g}$ are non-negative constants in any QFT. It is convenient to express eq. (2.5) in terms of new variables

$$
Y \equiv \frac{g^{2}}{y^{2}}, \quad x \equiv \ln \frac{g_{0}^{2}}{g^{2}}
$$

such that

$$
\frac{d Y}{d x}=A(Y-B)
$$

where

$$
A \equiv \frac{f_{g}}{b}-1, \quad B \equiv \frac{f_{y}}{f_{g}-b}
$$

\footnotetext{
${ }^{3}$ Theories where $b=0$ can have a different asymptotic solution for gauge couplings, $g^{2} \propto 1 / \sqrt{t}$, depending on the sign of the two-loop RGE coefficient for $g$. However such solution cannot be extended to systems with Yukawa and quartic couplings, where non-vanishing RGE arise at one loop.
} 
$A$ and $B$ have always the same sign: positive if $f_{g}>b$ and negative otherwise. The solution to eq. (2.7) is

$$
Y=\left(Y_{0}-B\right)\left(\frac{g_{0}^{2}}{g^{2}}\right)^{A}+B
$$

where $Y_{0}=g_{0}^{2} / y_{0}^{2}$ and $y_{0}$ is the Yukawa coupling at the IR scale $\mu_{0}(t=0)$. Equation (2.9) can be written more explicitly as

$$
y^{-2}=\left[y_{0}^{-2}-\frac{f_{y}}{\left(f_{g}-b\right) g_{0}^{2}}\right]\left(\frac{g_{0}^{2}}{g^{2}}\right)^{\frac{f_{g}}{b}}+\frac{f_{y}}{\left(f_{g}-b\right) g^{2}} .
$$

Landau poles in the Yukawa coupling exist if the equation $Y(t)=0$ admits solutions for some positive values of $t$. From eq. (2.9), we see that there are Landau poles when $A \leq 0$ or when $A>0$ and $B>Y_{0}$. Therefore, in the case of the Yukawa coupling, the conditions for TAF are $A>0$ and $B \leq Y_{0}$, which can be written explicitly as

$$
f_{g}>b \quad \text { and } \quad \frac{y_{0}^{2}}{g_{0}^{2}} \leq \frac{f_{g}-b}{f_{y}} \quad \text { (TAF conditions for the Yukawa coupling). }
$$

The one-loop coefficient $f_{g}$ is given by

$$
f_{g}=\frac{3}{2}\left(C_{2 \psi_{1}}+C_{2 \psi_{2}}\right)
$$

where $C_{2 \psi_{1,2}}$ are the quadratic Casimirs of the two fermions involved in the Yukawa coupling. The Yukawa coupling is allowed by TAF provided that their $C_{2}$ Casimirs are large enough. A gauge-neutral fermion ('right-handed neutrino') has $C_{2 \psi_{1}}=0$ : it can have Yukawa coupling compatibly with TAF provided that the other fermion has a large enough $C_{2 \psi_{2}}$.

Since $A>0$ for the TAF solution, the first term in eq. (2.9) dominates in the UV $(t \rightarrow \infty)$ over the constant term, as long as $B \neq Y_{0}$. Then, the asymptotic behaviour is

$$
y^{2} \propto\left(\frac{1}{t}\right)^{\frac{f g}{b}} \quad \text { for } t \rightarrow \infty
$$

and, at large $t$, the Yukawa coupling becomes negligible with respect to the gauge coupling, $g^{2} \propto 1 / t$. The exception (missed in [52]) is for $B=Y_{0}$, when $g$ and $y$ scale in the same way with $t$. We will refer to this case as fixed-flow, ${ }^{4}$ because couplings run, but their ratio is RG invariant. At the fixed-flow, the Yukawa coupling is fixed at any energy in terms of the gauge coupling

$$
y^{2}=\frac{f_{g}-b}{f_{y}} g^{2} \quad \text { for any } t .
$$

Equation (2.14) is an exact solution of the RGE, which corresponds to the PendletonRoss point [57] in the IR. We will see later that the existence of a fixed-flow solution for the Yukawa coupling is an important ingredient to satisfy the TAF requirement for scalar quartic couplings.

\footnotetext{
${ }^{4}$ We thank M. Strassler for suggesting this name to us.
} 

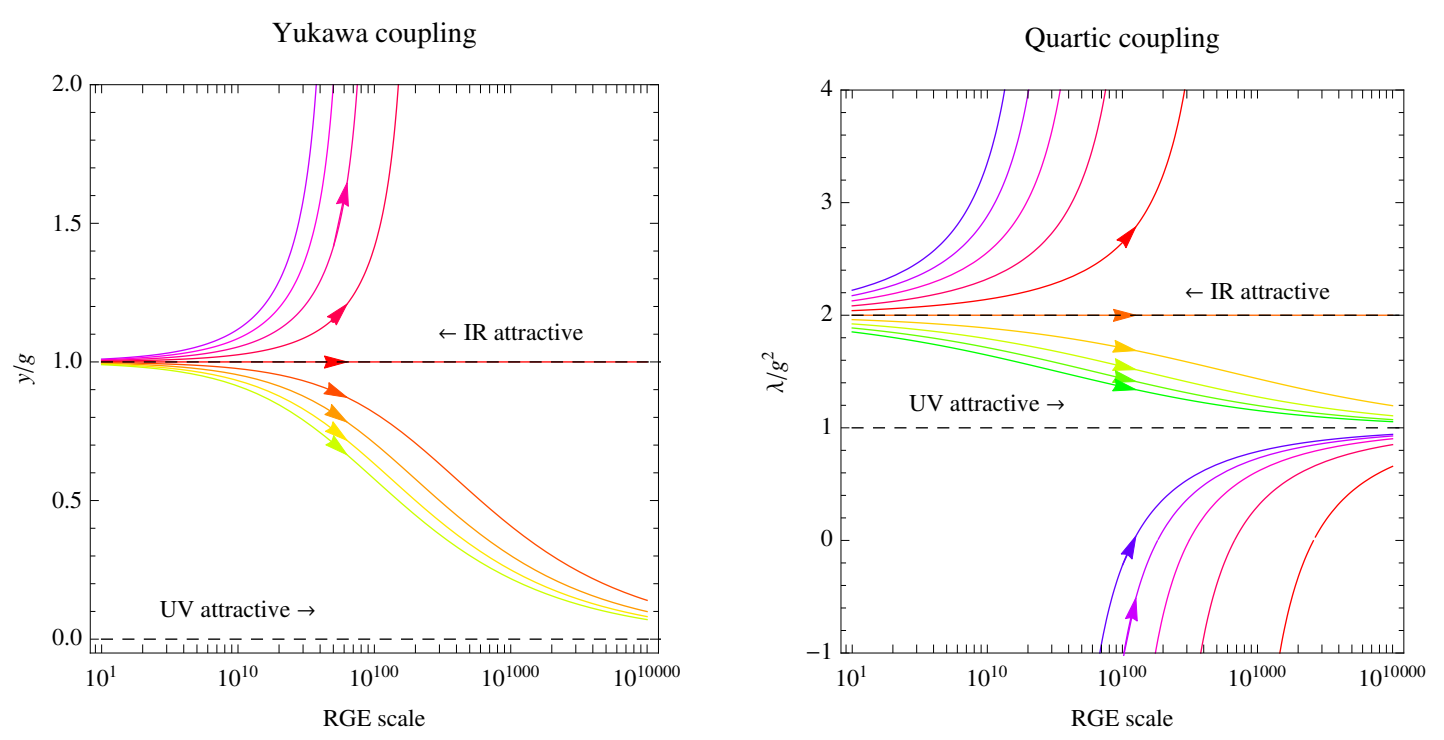

Figure 1. Sample of the general behaviour of the RG running of the Yukawa coupling $y$ (left, for $A=B=b=1$ ) and scalar quartic $\lambda$ (right, for $C=b=1, D=3 / 4, E=1 / 16$ ) varying the initial conditions.

We illustrate the situation for $A>0$ in figure 1 , where we plot the running of $y / g$ (= $1 / \sqrt{Y}$ ) for $A=1$ and $B=1$, while varying the initial condition of the coupling constants. The fixed-flow in eq. (2.14) corresponds to the limiting case of a family of solutions $\left(Y_{0} \leq B\right)$ that suffer from Landau poles; in the special case $Y_{0}=B$, the Landau pole slides to $t=\infty$ and the TAF condition is satisfied. The fixed-flow is an IR attractor. For $Y_{0}>B$, the solutions have the asymptotic behaviour in eq. (2.13) and are attracted in the UV to the point $y / g=0$.

Asymptotic solutions. It is instructive to obtain the asymptotic behaviour of the solutions of the RGE without using their complete analytic expression. This procedure is redundant here, because we have solved exactly the RGE, but it will be useful later, whenever we are not able to solve analytically the full RGE. Making the ansatz $Y=c t^{\alpha}$ for the asymptotic behaviour of the Yukawa coupling, eq. (2.7) turns into an algebraic equation

$$
c \alpha\left(1+\frac{1}{b g_{0}^{2} t}\right)=A\left(c-B t^{-\alpha}\right) \quad \text { for } \quad t \rightarrow \infty
$$

Asymptotic solutions can exist only for $\alpha \geq 0$, and we find two possibilities. Either $\alpha=A$, which corresponds to eq. (2.13); or $\alpha=0$ and $c=B$, which corresponds to eq. (2.14).

Moreover, the nature of the fixed-flow can be understood by analysing solutions of the form $Y=B+\Delta$, which represent small deformations of the fixed-flow solution. From eq. (2.7), we find that the perturbation $\Delta$ satisfies

$$
\frac{d}{d x}|\Delta|=A|\Delta|
$$


For $A>0$, the perturbation grows with $t$ and thus the fixed-flow is repulsive in the UV. So we can easily reproduce all the features of the asymptotic behaviour without solving analytically the RGE.

\subsection{One scalar quartic coupling}

Next we turn to the scalar quartic coupling, considering the case of a single coupling of each kind $(g, y, \lambda)$, where $g$ and $y$ are asymptotically free. The relevant RGE is

$$
\frac{d}{d t} \lambda=\lambda\left(s_{\lambda} \lambda+s_{\lambda y} y^{2}-s_{\lambda g} g^{2}\right)-s_{y} y^{4}+s_{g} g^{4}
$$

where, for any QFT, all coefficients $s_{i}$ are positive. We first solve eq. (2.17) in special cases.

One quartic, without Yukawa or gauge couplings. In this case, the solution to eq. (2.17) is

$$
\lambda^{-1}=\lambda_{0}^{-1}-s_{\lambda} t
$$

where $\lambda_{0}$ is the quartic coupling at the IR scale $\mu_{0}(t=0)$. If $\lambda_{0}>0$, a Landau pole is reached at the scale $t_{*}=1 /\left(\lambda_{0} s_{\lambda}\right)$. If $\lambda_{0}<0$, there are no Landau poles, but the coupling $\lambda$ is negative at all scales and the scalar potential is unstable. This means that other interactions, beyond the scalar quartic, are needed to satisfy TAF. This agrees with the well-known result [47-51] that no renormalisable field theory without non-abelian gauge fields can be asymptotically free.

One quartic, with one gauge coupling and no Yukawas. It is convenient to express the RGE eq. (2.17) in terms of new variables

$$
\Lambda \equiv \frac{g^{2}}{\lambda}, \quad x \equiv \ln \frac{g_{0}^{2}}{g^{2}}
$$

such that

$$
\frac{d \Lambda}{d x}=-C\left[(\Lambda-D)^{2}-E\right]
$$

where

$$
C \equiv \frac{s_{g}}{b}, \quad D \equiv \frac{s_{\lambda g}-b}{2 s_{g}}, \quad E \equiv \frac{\left(s_{\lambda g}-b\right)^{2}-4 s_{\lambda} s_{g}}{4 s_{g}^{2}} .
$$

Note that the definitions in eq. (2.21) imply $C>0$ and $D^{2}>E$. Thereby, the two special solutions where $\Lambda$ is RG invariant, $\Lambda(x)=\Lambda_{ \pm}=D \pm \sqrt{E}$ are both positive if $D>0$, and both negative otherwise. Since the right-hand side of eq. (2.20) depends only on the variable $\Lambda$, we can easily integrate the RGE.

For $E<0$, the solution is

$$
\Lambda=\frac{\Lambda_{0} \sqrt{-E}+\left[D\left(\Lambda_{0}-D\right)+E\right] \tan (C \sqrt{-E} x)}{\sqrt{-E}+\left(\Lambda_{0}-D\right) \tan (C \sqrt{-E} x)} \quad \text { for } E<0
$$

As the equation $\Lambda(x)=0$ admits solutions for positive $x$, the coupling $\lambda$ hits Landau poles. 
For $E>0$, the solution is

$$
\Lambda=\frac{(D+\sqrt{E})\left(\Lambda_{0}-D+\sqrt{E}\right)\left(\frac{g_{0}^{2}}{g^{2}}\right)^{2 C \sqrt{E}}-(D-\sqrt{E})\left(\Lambda_{0}-D-\sqrt{E}\right)}{\left(\Lambda_{0}-D+\sqrt{E}\right)\left(\frac{g_{0}^{2}}{g^{2}}\right)^{2 C \sqrt{E}}-\left(\Lambda_{0}-D-\sqrt{E}\right)} \text { for } E>0 .
$$

In this case, Landau poles (solutions of $\Lambda=0$ ) are found for $D<-\sqrt{E}$ or $D>\Lambda_{0}+\sqrt{E}$. Thus, the TAF requirement of having no Landau poles at any $t$ is $E \geq 0$ and $\sqrt{E} \leq D \leq$ $\Lambda_{0}+\sqrt{E}$ (recall that $|D|>\sqrt{E}$ ) or, more explicitly,

$$
s_{\lambda g}-b \geq 2 \sqrt{s_{\lambda} s_{g}} \text { and } \quad \frac{\lambda_{0}}{g_{0}^{2}} \leq \frac{\left(s_{\lambda g}-b\right)+\sqrt{\left(s_{\lambda g}-b\right)^{2}-4 s_{\lambda} s_{g}}}{2 s_{\lambda}}
$$

(TAF conditions for the scalar quartic coupling).

The solution in eq. (2.23) does not cross $\lambda=0$, whenever the TAF conditions in eq. (2.24) are satisfied. Therefore, there are no problems with potential stability at any $t$. The asymptotic behaviour of eq. (2.23) is $\Lambda=D+\sqrt{E}$, which can be written explicitly as

$$
\lambda=\frac{s_{\lambda g}-b-\sqrt{\left(s_{\lambda g}-b\right)^{2}-4 s_{\lambda} s_{g}}}{2 s_{\lambda} b t} \quad \text { for } t \rightarrow \infty .
$$

The asymptotic solution becomes an exact solution in the case $\Lambda_{0}=D+\sqrt{E}$. In this case we find a fixed-flow such that the ratio $\lambda / g^{2}$ is RG invariant. This corresponds to the condition

$$
\frac{\lambda}{g^{2}}=\frac{s_{\lambda g}-b-\sqrt{\left(s_{\lambda g}-b\right)^{2}-4 s_{\lambda} s_{g}}}{2 s_{\lambda}} \text { for any } t .
$$

As we approach $D=-\sqrt{E}$ or $\Lambda_{0}=D-\sqrt{E}$, the Landau pole slides to $t \rightarrow \infty$. The case $D=-\sqrt{E}$ is not interesting because it corresponds to $s_{\lambda}=0$, which is never verified in a QFT. Especially interesting is the case $D=\Lambda_{0}+\sqrt{E}$, in which the exact solution does not have the asymptotic behaviour of eq. (2.25). This case gives another kind of fixed-flow of the ratio $\lambda / g^{2}$, given by

$$
\frac{\lambda}{g^{2}}=\frac{s_{\lambda g}-b+\sqrt{\left(s_{\lambda g}-b\right)^{2}-4 s_{\lambda} s_{g}}}{2 s_{\lambda}} \text { for any } t .
$$

Note that, unlike the case of the Yukawa coupling, the quartic has only a single possible asymptotically-free behaviour, given by $\lambda \propto 1 / t$.

To visualise the situation for $E>0$, we show in figure $1 \mathrm{~b}$ the ratio $\lambda / g^{2}$, obtained from eq. (2.23), as a function of the RG parameter $t$ for different initial conditions $\Lambda_{0}$ (with the choice $C=b=1, D=3 / 4, E=1 / 16$, such that $\Lambda_{-}=1$ and $\left.\Lambda_{+}=2\right)$. The two fixed-flow solutions correspond to the two horizontal lines. The lower one, which is given by eq. (2.26), tracks the asymptotic behaviour of the other TAF solutions and therefore is a UV attractor. The upper one, given by eq. (2.27), corresponds to the separating case between a family of solutions (with $\Lambda_{0}>D-\sqrt{E}$ ) that blow up at finite $t$ and a family of asymptotically-free solutions (with $\Lambda_{0}<D-\sqrt{E}$ ). In the separating case (with $\Lambda_{0}=D-\sqrt{E}$ ), there is 
no Landau pole and the TAF condition can be satisfied. Therefore, the upper horizontal line, given by eq. (2.27), is an isolated solution that cannot be continuously deformed in the asymptotic UV region without violating the TAF conditions. It corresponds to an IR attractor.

Can the condition eq. (2.24) for TAF be satisfied, in absence of Yukawa couplings? Let us consider a generic scalar $\varphi$ in an irreducible representation $R$ with dimension $d_{R}$ and real dimension $d_{R R}\left(d_{R R}=d_{R}\right.$ for a real representation and $d_{R R}=2 d_{R}$ for a complex representation) with generators $T^{a}$ under a generic simple group $G$. We define the usual quadratic Casimirs as $\left(T^{a} T^{a}\right)_{i j}=C_{R} \delta_{i j}$ and $\operatorname{Tr}\left(T^{a} T^{b}\right)=\delta^{a b} T_{R}$, related by $d_{R} C_{R}=d_{A} T_{R}$, where $A$ is the adjoint representation. The RGE coefficient $s_{\lambda g}$ is given by $s_{\lambda g}=6 C_{R}$. To compute the other RGE coefficients, we suppose that the group theory allows only one quartic, given by the square of the quadratic, $V=\frac{1}{8} \lambda\left(\sum \varphi_{i}^{2}\right)^{2}$, where $\varphi_{i}$ are the canonically normalised $d_{R R}$ real components of $\varphi$. Then the other coefficients are

$$
s_{\lambda}=4+\frac{d_{R R}}{2}, \quad s_{g}=\frac{3 C_{R}\left[2 C_{R}\left(2 d_{A}+d_{R R}\right)-d_{A} C_{A}\right]}{d_{A}\left(2+d_{R R}\right)} .
$$

The TAF condition in eq. (2.24) explicitly becomes

$$
\left(C_{R}-\frac{b}{6}\right)^{2}>\frac{C_{R}}{6 d_{A}} \frac{8+d_{R R}}{2+d_{R R}}\left[2 C_{R}\left(d_{R R}+2 d_{A}\right)-6 d_{A} C_{A}\right] .
$$

In the most favourable case where the gauge $\beta$-function coefficient $b$ is small and can be neglected, the TAF condition simplifies to

$$
\frac{C_{A}}{C_{R}}>\frac{36}{8+d_{R R}}+2 \frac{d_{R R}}{d_{A}}-2 .
$$

Such condition favours representations smaller than the adjoint and large gauge groups. For $G=\mathrm{SU}(n)$ one has $C_{A}=n, d_{A}=n^{2}-1$ and, for its fundamental $n, T_{R}=1 / 2$ and $d_{R}=d_{R R} / 2=n$ such that $C_{R}=\left(n^{2}-1\right) / 2 n$. For $G=\operatorname{SO}(n)$ one has $C_{A}=(n-2) / 2$, $d_{A}=n(n-1) / 2$ and, for its fundamental $n, T_{R}=1 / 2$ and $d_{R}=d_{R R}=n$ such that $C_{R}=(n-1) / 4$. Therefore, in both cases the TAF condition is satisfied for $n$ larger than a critical value that depends on $b$.

The TAF condition for scalar quartics is more easily satisfied in presence of Yukawa couplings, as we now discuss.

One quartic, with one gauge and one Yukawa coupling. In terms of the variables introduced previously, eq. (2.17) for $g, y \neq 0$ becomes

$$
\frac{d}{d x} \Lambda=-\frac{1}{b}\left[\left(s_{g}-\frac{s_{y}}{Y^{2}}\right) \Lambda^{2}-\left(s_{\lambda g}-b-\frac{s_{\lambda y}}{Y}\right) \Lambda+s_{\lambda}\right] .
$$

Let us first consider the case in which the Yukawa is not on its fixed-flow. Although we cannot analytically integrate eq. (2.31), we can consider asymptotic solutions for $t \rightarrow \infty$. In this regime, $y$ is negligible with respect to $g$ and therefore the asymptotic solutions to eq. (2.31) are $\Lambda=\Lambda_{ \pm}$, i.e. the same as in the case without Yukawa, given by eqs. (2.26) 
and (2.27). The important difference is that, in this case, these solutions hold only in the $t \rightarrow \infty$ asymptotic region. The nature of these two asymptotic behaviours can be studied by making small deformations, taking $\Lambda=\Lambda_{ \pm}+\Delta_{ \pm}$. From eq. (2.31) we find that the perturbation $\Delta_{ \pm}$satisfies

$$
\frac{d}{d x}\left|\Delta_{ \pm}\right|= \pm \frac{\sqrt{\left(s_{\lambda g}-b\right)^{2}-4 s_{\lambda} s_{g}}}{b}\left|\Delta_{ \pm}\right|
$$

Thus, $\left|\Delta_{-}\right|$decreases at large $t$, while $\left|\Delta_{+}\right|$increases. This shows that $\Lambda_{-}$is UV attractive and $\Lambda_{+}$is UV repulsive. Also, $\Lambda_{+}$defines the divider between solutions with Landau poles and asymptotically-free solutions. The divider is an isolated solution that behaves as $\Lambda_{+}$ for $t \rightarrow \infty$. All other TAF solutions converge to $\Lambda_{-}$in the UV. These results are in agreement with what found in the previous section. In particular, the TAF conditions are still given by eq. (2.24).

Next, let us consider the case in which the Yukawa coupling is on its fixed-flow, given by eq. (2.14). Then eq. (2.31) becomes formally identical to eq. (2.20), after the replacement

$$
s_{g} \rightarrow s_{g}-\frac{\left(f_{g}-b\right)^{2}}{f_{y}^{2}} s_{y}, \quad s_{\lambda g} \rightarrow s_{\lambda g}-\frac{\left(f_{g}-b\right)}{f_{y}} s_{\lambda y} .
$$

Thus, the solutions to eq. (2.31) are analogous to those discussed in the previous section, once we replace the parameters $C, D, E$ defined in eq. (2.21) with

$$
\hat{C} \equiv \frac{1}{b}\left[s_{g}-\frac{\left(f_{g}-b\right)^{2}}{f_{y}^{2}} s_{y}\right], \quad \hat{D} \equiv \frac{1}{2 b \hat{C}}\left[s_{\lambda g}-\frac{\left(f_{g}-b\right)}{f_{y}} s_{\lambda y}-b\right], \quad \hat{E} \equiv \hat{D}^{2}-\frac{s_{\lambda}}{b \hat{C}}
$$

The important difference is that, unlike the case without Yukawa coupling, the parameter $\hat{C}$ can be either positive or negative. This gives rise to two classes of asymptotically-free solutions for the scalar quartic coupling.

The first class of solutions occurs for $\hat{C}>0$ (which implies $\hat{D}^{2}>\hat{E}$ ). The conditions for the absence of Landau poles are

$$
\hat{C}>0, \quad \hat{E}>0 \text { and } \frac{\lambda_{0}}{g_{0}^{2}} \leq(\hat{D}-\sqrt{\hat{E}})^{-1} \quad \begin{aligned}
& \text { (TAF conditions for the scalar } \\
& \text { quartic coupling with Yukawa } \\
& \text { on fixed-flow) }
\end{aligned}
$$

The exact solutions are given by eq. (2.23) (with the replacement $C, D, E \rightarrow \hat{C}, \hat{D}, \hat{E}$ ). From these solutions, we observe that the coupling $\lambda$ never crosses zero, so there is no instability issue. The asymptotic behaviour of these solutions for $t \rightarrow \infty$ is $\Lambda=\hat{D}+\sqrt{\hat{E}}$. In practical applications, the contribution from the Yukawa coupling on its fixed-flow can be very useful, because it is easier to satisfy the TAF conditions in eq. (2.35), rather than those in eq. (2.24).

The second class of solutions occurs for $\hat{C}<0$ (which implies $\hat{D}^{2}<\hat{E}$ ). The conditions for the absence of Landau poles are

$$
\hat{C}<0 \text { and } \frac{\lambda_{0}}{g_{0}^{2}} \leq(\hat{D}+\sqrt{\hat{E}})^{-1} \quad \begin{aligned}
& \text { (TAF conditions for the scalar quartic } \\
& \text { coupling with Yukawa on fixed-flow) } .
\end{aligned}
$$


The exact solutions are given again by eq. (2.23) (with the replacement $C, D, E \rightarrow \hat{C}, \hat{D}, \hat{E}$ ). In this case, if the coupling $\lambda$ starts positive in the IR, it will cross zero, becoming negative at high energy and raising a problem with the stability of the potential. The asymptotic behaviour of the solutions for $t \rightarrow \infty$ is $\Lambda=\hat{D}-\sqrt{\hat{E}}$.

\subsection{Multiple gauge couplings}

The generalisation to semi-simple groups is trivial, since in the one-loop approximation each gauge coupling evolves independently

$$
\frac{d}{d t} g_{i}^{2}=-b_{i} g_{i}^{4}
$$

Here the index $i$ scans over the couplings of the different simple gauge group factors. The full solutions are $g_{i}^{2}=g_{0 i}^{2} /\left(1+g_{0 i}^{2} b_{i} t\right)=1 / b_{i}\left(t-t_{i}\right)$, where $t_{i}=-1 / b_{i} g_{0 i}^{2}$ are the low-energy scales where the gauge coupling $g_{i}$ becomes non-perturbative. The asymptotic behaviour is

$$
g_{i}^{2} \simeq \frac{1}{b_{i} t} \quad \text { for } t \rightarrow \infty
$$

The TAF conditions are

$$
b_{i}>0 \quad \text { (TAF conditions for multiple gauge couplings). }
$$

One Yukawa, with multiple gauge couplings. We consider the case of a single Yukawa coupling with a semi-simple gauge group. Equation (2.5) becomes

$$
\frac{d}{d t} y^{2}=y^{2}\left(f_{y} y^{2}-\sum_{i} f_{g_{i}} g_{i}^{2}\right)
$$

where $f_{y}$ and $f_{g_{i}}$ are non-negative constants in any QFT. The general solution of eq. (2.40), analogous to eq. (2.10), is

$$
y^{-2}=\left(y_{0}^{-2}-f_{y} I\right) \prod_{i}\left(\frac{g_{i 0}^{2}}{g_{i}^{2}}\right)^{\frac{f_{g_{i}}}{b_{i}}}
$$

where

$$
I(t) \equiv \int_{0}^{t} d t^{\prime} \prod_{i}\left(\frac{g_{i 0}^{2}}{g_{i}^{2}}\right)^{-\frac{f g_{i}}{b_{i}}} .
$$

When $\sum_{i} f_{g_{i}} / b_{i} \leq 1$, eq. (2.41) always encounters a Landau pole. For $\sum_{i} f_{g_{i}} / b_{i}>1$, we can define $I_{\infty} \equiv \lim _{t \rightarrow \infty} I(t)$ and expand $I$ in the asymptotic region as

$$
I(t)=I_{\infty}+\frac{\prod_{i}\left(g_{i 0}^{2} b_{i}\right)^{-\frac{f g_{i}}{b_{i}}}}{1-\sum_{i} \frac{f_{g_{i}}}{b_{i}}} t^{1-\sum_{i} \frac{f g_{i}}{b_{i}}} \quad \text { for } t \rightarrow \infty
$$

Then, the conditions for TAF, which generalise eq. (2.11), are

$$
\sum_{i} \frac{f_{g_{i}}}{b_{i}}>1 \text { and } y_{0}^{2} \leq \frac{1}{f_{y} I_{\infty}} \quad \text { (TAF condition for the Yukawa, with multiple } g_{i} \text { ). }
$$


Whenever the second condition in eq. (2.44) is satisfied as a strict inequality, the asymptotic behaviour is

$$
y^{2}=\frac{t^{-\sum_{i} \frac{f g_{i}}{b_{i}}}}{\left(y_{0}^{-2}-f_{y} I_{\infty}\right) \prod_{i}\left(g_{i 0}^{2} b_{i}\right)^{\frac{f g_{i}}{b_{i}}}} \quad \text { for } t \rightarrow \infty
$$

and the Yukawa coupling decreases faster than the gauge couplings at large $t$. However, when $y_{0}^{-2}=f_{y} I_{\infty}$, the ratio between the Yukawa coupling and any gauge coupling $g_{j}$ is constant in the asymptotic region,

$$
\frac{y^{2}}{g_{j}^{2}}=\frac{b_{j}}{f_{y}}\left[\left(\sum_{i} \frac{f_{g_{i}}}{b_{i}}\right)-1\right] \quad \text { for } t \rightarrow \infty .
$$

Unlike the case of a single gauge coupling, in which the ratio $y^{2} / g^{2}$ was RG invariant on the fixed-flow, eq. (2.46) is valid only in the asymptotic regime. It corresponds to an isolated RGE solution, which behaves as an attractor in the IR and is characterised by the low-energy value $y_{0}^{2}=1 / f_{y} I_{\infty}$.

One quartic, with one Yukawa and multiple gauge couplings. In this case the RGE for the scalar quartic coupling $\lambda$ is

$$
\frac{d}{d t} \lambda=\lambda\left(s_{\lambda} \lambda+s_{\lambda y} y^{2}-\sum_{i} s_{\lambda_{g i}} g_{i}^{2}\right)-s_{y} y^{4}+\sum_{i j} s_{g i j} g_{i}^{2} g_{j}^{2}
$$

where $s_{\lambda}, s_{\lambda y}, s_{\lambda g i}, s_{y}$ and $s_{g i j}$ are non-negative constants.

Although we do not solve exactly eq. (2.47), we can easily obtain the asymptotic behaviour for $t \rightarrow \infty$. When the Yukawa coupling does not satisfy the special initial condition $y_{0}^{2}=1 / f_{y} I_{\infty}$, it can be neglected with respect to the gauge couplings in the deep UV. Then, the two possible asymptotic behaviours for the scalar quartic are

$$
\begin{gathered}
\frac{g_{k}^{2}}{\lambda}=D_{k} \pm \sqrt{E_{k}} \quad \text { for } t \rightarrow \infty, \\
C_{k} \equiv b_{k} \sum_{i, j} \frac{s_{g_{i j}}}{b_{i} b_{j}}, \quad D_{k} \equiv \frac{1}{2 C_{k}}\left(\sum_{i} \frac{s_{\lambda g_{i}}}{b_{i}}-1\right), \quad E_{k} \equiv D_{k}^{2}-\frac{s_{\lambda}}{b_{k} C_{k}} .
\end{gathered}
$$

The parameters $C_{k}, D_{k}, E_{k}$ are the generalisation to multiple-gauge couplings of the parameters $C, D, E$ previously defined in eq. (2.21). Indeed, the discussion of the solutions is completely analogous to the case of a single gauge coupling, once we translate the parameters $C, D, E$ into $C_{k}, D_{k}, E_{k}$. In particular $E_{k}>0$ is a necessary condition for TAF.

When the Yukawa coupling satisfies $y_{0}^{2}=1 / f_{y} I_{\infty}$, it has the same asymptotic behaviour as the gauge couplings in the UV. In this case, the asymptotic solutions for the scalar 
quartic are

$$
\begin{aligned}
& \frac{g_{k}^{2}}{\lambda}=\hat{D}_{k} \pm \sqrt{\hat{E}_{k}} \quad \text { for } t \rightarrow \infty \\
& \hat{C}_{k} \equiv b_{k}\left[\sum_{i, j} \frac{s_{g_{i j}}}{b_{i} b_{j}}-\left(\sum_{i} \frac{f_{g_{i}}}{b_{i}}-1\right)^{2} \frac{s_{y}}{f_{y}^{2}}\right], \\
& \hat{D}_{k} \equiv \frac{1}{2 \hat{C}_{k}}\left[\sum_{i} \frac{s_{\lambda g_{i}}}{b_{i}}-\left(\sum_{i} \frac{f_{g_{i}}}{b_{i}}-1\right) \frac{s_{\lambda y}}{f_{y}}-1\right], \quad \hat{E}_{k} \equiv \hat{D}_{k}^{2}-\frac{s_{\lambda}}{b_{k} \hat{C}_{k}} .
\end{aligned}
$$

Again, the discussion of the solutions is analogous to the case of a single gauge coupling, with the translation of the parameters $\hat{C}, \hat{D}, \hat{E}$ defined in eq. (2.34) into the multiple-gauge parameters $\hat{C}_{k}, \hat{D}_{k}, \hat{E}_{k}$.

\subsection{Supersymmetric case}

In the context of a generic QFT, having the Yukawa coupling sitting exactly on the fixedflow solution of the kind of eq. (2.14), with the isolated asymptotic behaviour $y^{2} \propto 1 / t$, may seem a very special situation corresponding to an extreme fine-tuning of the initial condition for the $y / g$ ratio. It is interesting to remark that, in the context of supersymmetric theories, such a special situation could be dictated by symmetry properties. The Yukawa coupling $y_{g}$ of the fermion-sfermion-gaugino interaction automatically satisfies the condition to sit on the fixed-flow. This is because supersymmetry ensures that $y_{g}$ is proportional to the gauge coupling $g$ at all scales. On the contrary, as seen in section 2.4, when the Yukawa coupling of a generic QFT is on its fixed-flow, the proportionality between $y$ and $g$ holds only in the asymptotic region $t \rightarrow \infty$, while a more complicated behaviour appears at finite energy.

The other interesting aspect about supersymmetry is that scalar quartic couplings are induced by $D$-terms and therefore are proportional to $g^{2}$. Gauge asymptotic freedom automatically ensures that such quartic couplings are free from Landau poles and satisfy the TAF conditions. Nevertheless, low-energy supersymmetry does not offer much practical advantage in the construction of TAF extensions of the SM. The reason is that, in lowenergy supersymmetry, both $\mathrm{U}(1)_{Y}$ and $\mathrm{SU}(2)_{L}$ are non-asymptotically free and a TAF extension requires rather big gauge groups at the weak scale.

\section{Conditions for TAF: general case}

In section 2 we analysed the RGE in some simple cases, finding analytic solutions. However, as soon as we increase the number of couplings, the problem of solving exactly the RGE quickly becomes analytically cumbersome and intractable. Armed with the experience acquired from the simple cases, we can now present a systematic procedure to identify all TAF conditions in a generic QFT with multiple couplings. In this section we will illustrate this method.

We consider a generic QFT with multiple gauge couplings $g_{i}$, Yukawa couplings $y_{a}$ and scalar quartic couplings $\lambda_{m}$. Our starting point is to define new rescaled couplings 
$x_{I}=\left\{\tilde{g}_{i}, \tilde{y}_{a}, \tilde{\lambda}_{m}\right\}$ by factoring out the leading asymptotic behaviour $1 / t$ :

$$
g_{i}^{2}(t)=\frac{\tilde{g}_{i}^{2}(t)}{t}, \quad y_{a}^{2}(t)=\frac{\tilde{y}_{a}^{2}(t)}{t}, \quad \lambda_{m}(t)=\frac{\tilde{\lambda}_{m}(t)}{t}
$$

where $t=\ln \left(\mu^{2} / \mu_{0}^{2}\right) /(4 \pi)^{2}$. The one-loop RGE for the couplings $x_{I}$ are

$$
\frac{d \tilde{g}_{i}}{d \ln t}=\frac{\tilde{g}_{i}}{2}+\beta_{g_{i}}(\tilde{g}), \quad \frac{d \tilde{y}_{a}}{d \ln t}=\frac{\tilde{y}_{a}}{2}+\beta_{y_{a}}(\tilde{g}, \tilde{y}), \quad \frac{d \tilde{\lambda}_{m}}{d \ln t}=\tilde{\lambda}_{m}+\beta_{\lambda_{m}}(\tilde{g}, \tilde{y}, \tilde{\lambda}) .
$$

The right-hand sides of the RGE in eq. (3.2) do not depend explicitly on $t$, but only through the functional dependence of the couplings $x_{I}$. This is true in the one-loop approximation, because $\beta_{g_{i}}$ is cubic in $g ; \beta_{y_{a}}$ is cubic in $g$ and $y ; \beta_{\lambda_{m}}$ is quadratic in $g^{2}, y^{2}, \lambda$. As discussed at the beginning of section 2, the one-loop approximation is adequate for our purposes. Thus, the RGE in eq. (3.2) take the form of a vector flow in the space of the rescaled $x$ couplings,

$$
\frac{d x_{I}}{d \ln t}=V_{I}(x), \quad x_{I}=\left\{\tilde{g}_{i}, \tilde{y}_{a}, \tilde{\lambda}_{m}\right\}
$$

The next step of the procedure is to identify the possible asymptotic behaviours by solving the system of algebraic equations

$$
V_{I}\left(x_{\infty}\right)=0 \Rightarrow\left\{\begin{array}{l}
\tilde{g}_{i \infty}=-2 \beta_{g_{i}}\left(\tilde{g}_{\infty}\right) \\
\tilde{y}_{a \infty}=-2 \beta_{y_{a}}\left(\tilde{g}_{\infty}, \tilde{y}_{\infty}\right) \\
\tilde{\lambda}_{m \infty}=-\beta_{\lambda_{m}}\left(\tilde{g}_{\infty}, \tilde{y}_{\infty}, \tilde{\lambda}_{\infty}\right)
\end{array}\right.
$$

The constants $x_{\infty}=\left\{\tilde{g}_{i \infty}, \tilde{y}_{a \infty}, \tilde{\lambda}_{m \infty}\right\}$ are fixed points of the RG flow for the rescaled couplings $x$. We will call them fixed-flows, extending the terminology introduced in section 2, since they describe special RG trajectories in which individual couplings run, but their ratio is fixed. The constants $x_{\infty}$ correspond to RG solutions for the running couplings with infinite boundary conditions at the IR scale $t=0$. These solutions are especially useful to track the asymptotic behaviour of the RG running at $t \rightarrow \infty$. When one of the $x_{\infty}$ constants vanishes, it can either mean that the corresponding running coupling vanishes (if the fixed-flow is UV-repulsive) or that there are running coupling solutions with subleading asymptotic behaviour $t^{-\alpha}$ with $\alpha>1$ (if the fixed-flow is UV-attractive).

The main qualitative characterisation of each fixed-flow is its UV-attractive or repulsive behaviour. The nature of each fixed-flow can be understood by linearising eq. (3.3) in the neighbourhood of $x=x_{\infty}$, where the vector flow is approximated by

$$
V_{I}(x) \simeq \sum_{J} M_{I J}\left(x_{J}-x_{J \infty}\right), \quad \text { where } \quad M_{I J}=\left.\frac{\partial V_{I}}{\partial x_{J}}\right|_{x=x_{\infty}}
$$

is a numerical matrix. The RGE for the small deformation around the fixed-flow solution, $\Delta_{I} \equiv x_{I}-x_{I \infty}$, is

$$
\frac{d \Delta_{I}}{d \ln t}=\sum_{J} M_{I J} \Delta_{J} .
$$


From eq. (3.6) we obtain the following result on the nature of the solutions. A fixed-flow $x_{\infty}$ is fully $U V$-attractive (IR-repulsive) if all the eigenvalues of the matrix $M\left(x_{\infty}\right)$ are negative, and is fully UV-repulsive (IR-attractive) if all eigenvalues are positive.

A UV-repulsive fixed-flow corresponds to an isolated asymptotic behaviour: a small deviation from the fixed-flow will bring the solution further away as we move towards the UV, and therefore will lead to a different asymptotic behaviour. This means that the request of sitting on a UV-repulsive fixed-flow implies a precise determination of one combination of couplings in the IR.

In general, some of the eigenvalues of the matrix $M\left(x_{\infty}\right)$ are positive, and others are negative. The number of positive eigenvalues is the number of combinations of couplings that are univocally predicted by demanding that the theory can reach infinite energy at that fixed-flow. The number of negative eigenvalues is the residual number of free parameters. ${ }^{5}$

The matrix $M\left(x_{\infty}\right)$ has constant entries that depend only on $x_{\infty}$ and is given by

$$
M\left(x_{\infty}\right)=\left(\begin{array}{ccc}
\delta_{i j}\left(\frac{1}{2}-\frac{3}{2} b_{i} \tilde{g}_{i}^{2}\right) & 0 & 0 \\
\frac{\partial \beta_{y_{a}}(x)}{\partial \tilde{g}_{j}} & \frac{\delta_{a b}}{2}+\frac{\partial \beta_{y_{a}}(x)}{\partial \tilde{y}_{b}} & 0 \\
\frac{\partial \beta_{\lambda_{m}}(x)}{\partial \tilde{g}_{j}} & \frac{\partial \beta_{\lambda_{m}}(x)}{\partial \tilde{y}_{b}} & \delta_{m n}+\frac{\partial \beta_{\lambda_{m}}(x)}{\partial \tilde{\lambda}_{n}}
\end{array}\right)_{x=x_{\infty}}
$$

where $b_{i}$ are the gauge $\beta$-function coefficients. The matrix is block triangular. This means that the nature of the gauge fixed-flows are not influenced by Yukawa and quartic couplings, and the nature of the Yukawa fixed-flows are not influenced by quartic couplings. This follows from the consideration that the eigenvalues of any triangular matrix are equal to its diagonal elements (as can be easily proved by induction). The special structure of the matrix $M\left(x_{\infty}\right)$ suggests that we can proceed in steps, solving in succession the cases of gauge, Yukawa, and scalar quartic couplings. In each case, we first solve the system of equations (3.4), $V_{I}\left(x_{\infty}\right)=0$, to determine the fixed-flows and then, for each solution, we compute the eigenvalues of the matrix $M\left(x_{\infty}\right)$ in eq. (3.7) to determine their $U V$-attractive or repulsive nature.

\subsection{Gauge couplings}

For each gauge coupling, we find at most two fixed-flows that solve eq. (3.4):

$$
\begin{aligned}
& \text { for } b_{i}>0 \quad \tilde{g}_{i \infty}^{2}= \begin{cases}1 / b_{i} & \text { UV-attractive } \\
0 & \text { UV-repulsive }\end{cases} \\
& \text { for } b_{i}<0 \quad \tilde{g}_{i \infty}^{2}=0 \quad \text { UV-repulsive }
\end{aligned}
$$

The solution with $\tilde{g}_{i \infty} \neq 0$, being UV-attractive, does not imply any prediction for the value of the gauge coupling in the IR, and exists only when the gauge group is asymptotically free, $b_{i}>0$. If this is not the case, a vanishing gauge coupling is the only way to achieve TAF.

\footnotetext{
${ }^{5}$ Zero eigenvalues are also possible, and correspond to accidental global flavour symmetries of the theory (an example is discussed in section 5). Imaginary eigenvalues are absent in all the examples that we computed, which means that the asymptotic RGE flow never performs cycles around a fixed point.
} 
The solution $\tilde{g}_{i \infty}=0$ is always present and, being UV-repulsive, implies an IR prediction, which is $g_{i 0}=0$. Of course, these results are trivial and agree with what can be easily derived from the explicit solutions of eq. (2.37).

\subsection{Yukawa couplings}

Next, let us consider the Yukawa couplings $y_{a}$, whose one-loop RGE are

$$
\frac{d}{d t} y_{a}=\beta_{y_{a}}(g, y)=\frac{1}{2}\left(f_{a b c d}^{y} y_{b} y_{c} y_{d}-f_{i a}^{g} g_{i}^{2} y_{a}\right)
$$

where $f^{y}$ and $f^{g}$ are numerical coefficients. In the general case, eq. (3.10) cannot be analytically solved and our method becomes essential to analyse the problem. For the Yukawa couplings eq. (3.4), which determines the fixed-flows, becomes

$$
\tilde{y}_{a \infty}=-2 \beta_{y_{a}}\left(b^{-1 / 2}, \tilde{y}_{\infty}\right)=-f_{a b c d}^{y} \tilde{y}_{b \infty} \tilde{y}_{c \infty} \tilde{y}_{d \infty}+\frac{f_{i a}^{g}}{b_{i}} \tilde{y}_{a \infty} .
$$

For illustration, we can solve eq. (3.11) in the case of a single Yukawa and gauge coupling:

$$
\begin{aligned}
& \text { for } f_{g}>b \quad \tilde{y}_{\infty}^{2}= \begin{cases}\left(f_{g}-b\right) /\left(b f_{y}\right) & \text { UV-repulsive } \\
0 & \text { UV-attractive }\end{cases} \\
& \text { for } f_{g}<b \quad \tilde{y}_{\infty}^{2}=0 \quad \text { UV-repulsive. }
\end{aligned}
$$

Comparing eq. (3.12) with eq. (3.8) for $f_{g}>b>0$, we see that the nature of the nonvanishing $\left(\tilde{y}_{\infty} \neq 0\right)$ and vanishing $\left(\tilde{y}_{\infty}=0\right)$ fixed-flows of the Yukawa coupling is reversed with respect to the case of the gauge coupling. So, in the neighbourhood of $\tilde{y}_{\infty}=0$, we expect a family of solutions with an asymptotic behaviour subleading with respect to $1 / t$. On the other hand, the non-vanishing fixed-flow corresponds to an isolated asymptotic behaviour $y^{2}=\tilde{y}_{\infty}^{2} / t$ and leads to a prediction for a combination of couplings in the IR. As derived in section 2 , the IR prediction is $y_{0}^{2} / g_{0}^{2}=\left(f_{g}-b\right) / f_{y}$ in the case of a single gauge coupling and $y_{0}^{-2}=f_{y} I_{\infty}$ in the case of multiple couplings, where $I_{\infty}$ is defined after eq. (2.42).

Going back to the general case, the task is to find the set of real solutions of the system of cubic equations (3.11). The problem is often simplified by the following observation: $\beta_{y_{a}}$ vanishes for $y_{a}=0$ whenever the Lagrangian acquires a chiral symmetry in the limit $y_{a}=0$. In such a case, one of the fixed-flows is simply $\tilde{y}_{a \infty}=0$ (and is UV-attractive for $\sum_{i} f_{i a}^{g} / b_{i}>1$, as can be seen by computing the matrix $M$ ). The other fixed-flow is found by solving a linear equation in $\tilde{y}_{a \infty}^{2}$ and choosing the positive value of the Yukawa coupling. If a chiral symmetry holds for each one of the $N_{y}$ Yukawa couplings, the full cubic system is reduced to a linear system that admits up to $2^{N_{y}}$ solutions. This simplification holds in various theories of interest, when the number $N_{y}$ of Yukawa couplings is less than the number of fermionic fields (with associated chiral symmetries). In theories with multiple generations one has a continuum of solutions, trivially obtained by acting on one representative solution with the flavour symmetry of the theory. 


\subsection{Scalar quartic couplings}

The one-loop RGE for the scalar quartic couplings $\lambda_{m}$ is

$$
\frac{d}{d t} \lambda_{m}=\beta_{\lambda_{m}}(g, y, \lambda)=s_{m n p}^{\lambda} \lambda_{n} \lambda_{p}+\lambda_{m}\left(s_{m a b}^{\lambda y} y_{a} y_{b}-s_{m i}^{\lambda g} g_{i}^{2}\right)-s_{m a b c d}^{y} y_{a} y_{b} y_{c} y_{d}+s_{m i j}^{g} g_{i}^{2} g_{j}^{2} .
$$

The fixed-flows are obtained by solving eq. (3.4),

$$
\tilde{\lambda}_{m \infty}=-\beta_{\lambda_{m}}\left(b^{-1 / 2}, \tilde{y}_{\infty}, \tilde{\lambda}_{\infty}\right),
$$

where $\tilde{y}_{\infty}$ are the solutions of eq. (3.11).

For illustration, we can solve eq. (3.15) in the case of a single quartic, Yukawa, and gauge couplings. We find that the fixed-flows are

$$
\tilde{\lambda}_{\infty}=\tilde{\lambda}_{ \pm} \equiv \frac{s_{\lambda g}-b-s_{\lambda y} b \tilde{y}_{\infty}^{2} \pm \sqrt{\left(s_{\lambda g}-b-s_{\lambda y} b \tilde{y}_{\infty}^{2}\right)^{2}-4 s_{\lambda}\left(s_{g}-s_{y} b^{2} \tilde{y}_{\infty}^{4}\right)}}{2 s_{\lambda} b},
$$

where the two possible values of $\tilde{y}_{\infty}$ are given in eq. (3.12). The solutions $\tilde{\lambda}_{ \pm}$exist only under the condition that the term inside the square root in eq. (3.16) is not negative. Inspection of the matrix $M\left(x_{\infty}\right)$ in eq. (3.7) shows that $\tilde{\lambda}_{+}$is UV-repulsive, while $\tilde{\lambda}_{-}$ is UV-attractive. Since both fixed-flows are non-vanishing, they correspond to running couplings with the same asymptotic behaviour $\lambda \sim 1 / t$. The request of sitting exactly on the UV-repulsive fixed-flow $\tilde{\lambda}_{+}$implies an IR prediction, which is $\lambda_{0} / g_{0}^{2}=b \tilde{\lambda}_{+}$in one-loop approximation. These conclusions are in agreement with the full analytic study of the RGE presented in section 2.3 .

In general, eq. (3.15) is a system of quadratic equations in $\tilde{\lambda}_{m \infty}$, for any given $\tilde{y}_{a \infty}$ and $\tilde{g}_{i \infty}$. The TAF conditions are obtained by requiring that at least one such system admits a solution where all coefficients $\tilde{\lambda}_{m \infty}$ are real. Usually $\tilde{\lambda}_{m \infty}=0$ is not a solution, because of the additive renormalisation of quartic couplings due to gauge and Yukawa couplings. In some models, a quartic coupling can break an accidental global symmetry: only in such a case $\tilde{\lambda}_{m \infty}=0$ is a solution, providing an easy way to find the other solution.

\subsection{Basins of attraction}

The study of the fixed-flows determines the asymptotic behaviour of the RG trajectories and the necessary conditions on the field content of the theory to satisfy TAF. The next step of our procedure consists in determining the initial conditions of the coupling constants in the IR that insure that all couplings flow towards a vanishing fixed-point in the far UV, without being attracted towards a Landau pole at finite $t$. This is done by studying the basins of attraction of the fixed-flows in the space of the rescaled couplings $x_{I}=\left\{\tilde{g}_{i}, \tilde{y}_{a}, \tilde{\lambda}_{m}\right\}$, defined as the parameter range covered by stream lines that flow into such point. Every positive eigenvalue of $M$ implies a reduced dimensionality of such parameter space, and thereby one prediction for a combination of couplings.

As an example, we show in figure 2 at page 24 the flow $V_{I}(x)$ and the fixed points $x=x_{\infty}$ for the SM with vanishing hypercharge gauge coupling, a case which will be 
discussed in detail in section 4 . The basin of attraction of the fully UV-attractive fixedflow (in blue) is the shaded (two-dimensional) region. The basins of attraction of the mixed IR/UV fixed-flows (in magenta) are the magenta curves: one parameter is predicted and the region is one-dimensional. The basin of the fully UV-repulsive fixed-flow (in red) is the point itself: two couplings are predicted and the region is zero-dimensional.

The RG flow of the scalar quartic couplings can cross the boundary that separates a stable from an unstable potential. When a UV-repulsive fixed-flow corresponds to a stable potential and a UV-attractive fixed-flow corresponds to an unstable potential, the low-energy vacuum is meta-stable. An example of such a situation is shown in figure $2 \mathrm{~b}$, in which there is a single quartic $\lambda$, and the stability condition is $\lambda>0$. In the opposite situation (which is possible with multiple quartics) a stable potential can become unstable at low energy, signalling that the phenomenon of spontaneous symmetry breaking is taking place, according to the Coleman-Weinberg mechanism [58].

\subsection{Mass parameters}

So far we have focused our discussion on the RG running of dimensionless parameters, i.e. the coupling constants of the theory. Mass parameters (scalar and fermion masses and cubic scalar couplings) become dynamically irrelevant at very high energy and so are not crucial for our considerations. Nevertheless, for completeness, we discuss now their RG evolution.

Let us first consider the simplest case of a theory with a single mass parameter $m$ for a scalar field. The one-loop RGE can be easily solved

$$
\frac{d m^{2}}{d t}=\gamma m^{2}, \quad \Rightarrow \quad m^{2}(t)=m^{2}\left(t_{0}\right) \exp \int_{t_{0}}^{t} \gamma(t) d t
$$

Here $\gamma$ is the anomalous dimension which, in the case of the SM Higgs, is given by

$$
\gamma=3 y_{t}^{2}+6 \lambda-\frac{9}{4}\left(g_{2}^{2}+\frac{g_{1}^{2}}{5}\right) .
$$

In a TAF theory, the leading asymptotic behaviour is $\gamma(t)=\tilde{\gamma} / t$, where $\tilde{\gamma}$ is a constant given by the fixed-flows of the coupling constants (for instance, as discussed in section 4 , in the TAF version of the SM with $g_{1}=0$, we find $\left.\tilde{\gamma} \approx-0.75\right)$. Therefore, the asymptotic RG behaviour of $m^{2}$ is

$$
m^{2}(t)=m^{2}\left(t_{0}\right) \times\left(t / t_{0}\right)^{\tilde{\gamma}} .
$$

For negative $\tilde{\gamma}$, the mass parameter $m^{2}$ flows to zero in the UV. For positive $\tilde{\gamma}$, there is an infinite multiplicative renormalisation as $t \rightarrow \infty$ (although $m^{2}(t)$ remains always negligible with respect to the renormalisation scale $\mu^{2}$ in the UV).

In ref. [46], it is claimed that this infinite renormalisation introduces a hierarchy problem, even for asymptotically-free theories. We disagree with this conclusion. In an asymptotically-free theory with a single scalar mass, there are no physical mass scales larger than $m$. Therefore, no hierarchies can arise and the theory is natural.

The issue of naturalness comes up in theories with multiple mass scales. Let us consider a TAF model with two very different scalar mass parameters $m_{1}^{2}$ and $m_{2}^{2}$. The RG trajectory 
of the smallest of the two masses will start in the IR with a logarithmic running, until it meets the second mass scale, where it receives a threshold correction proportional to the large mass. At that scale, the RG trajectory has a sudden jump, and then follows a logarithmic running proportional to the heavy mass. This special RG trajectory is very sensitive to initial conditions and a large separation between $m_{1}^{2}$ and $m_{2}^{2}$ requires a careful tuning of parameters. The instability of the RG trajectory for very different $m_{1}^{2}$ and $m_{2}^{2}$ is a reincarnation of the naturalness problem. On the other hand, the RG trajectory in eq. (3.19) does not exhibit any special sensitivity on initial conditions, confirming our conclusion that there is no naturalness problem in a theory with a single mass scale.

It is interesting to consider the RG flow towards the UV of the TAF theory with two mass scales. Given the RG evolution of the mass parameters $d m_{i}^{2} / d t=\gamma_{i j} m_{j}^{2}$, the ratio $r=m_{1}^{2} / m_{2}^{2}$ obeys the equation

$$
\frac{d r}{d t}=\gamma_{12}+\left(\gamma_{11}-\gamma_{22}\right) r-\gamma_{21} r^{2}
$$

In the asymptotic region, we can write $\gamma_{i j}(t)=\tilde{\gamma}_{i j} / t$ with constant $\tilde{\gamma}_{i j}$ determined by the fixed-flows of the coupling constants. In the special case $\gamma_{12}=\gamma_{21}=0$, the two scalar particles belong to two different sectors with no common interactions and $r=r_{0}\left(t / t_{0}\right)^{\tilde{\gamma}_{11}-\tilde{\gamma}_{22}}$. Each mass parameter evolves independently and, for $t \rightarrow \infty, r$ flows to zero (when $\tilde{\gamma}_{22}>\tilde{\gamma}_{11}$ ) or to infinity (when $\tilde{\gamma}_{11}>\tilde{\gamma}_{22}$ ).

On the other hand, when there are common interactions between the two scalars $\left(\gamma_{12}, \gamma_{21} \neq 0\right)$, the ratio $r$ can have a more complicated evolution and, in particular, cross $r=0$ or $1 / r=0$ at finite $t$, leading to a dynamical generation of spontaneous symmetry breaking. When $\gamma_{12}$ and $\gamma_{21}$ are positive (which is always true for scalars with mutual quartic and trilinear interactions, but does not hold when there are other sources in the RGE from large masses of vector-like fermions), the asymptotic solution of eq. (3.20) is ${ }^{6}$

$$
r(t)=\frac{r_{+}\left(r_{0}-r_{-}\right)\left(t / t_{0}\right)^{\Delta}-r_{-}\left(r_{0}-r_{+}\right)}{\left(r_{0}-r_{-}\right)\left(t / t_{0}\right)^{\Delta}-\left(r_{0}-r_{+}\right)},
$$

where

$$
r_{ \pm} \equiv \frac{\tilde{\gamma}_{11}-\tilde{\gamma}_{22} \pm \Delta}{2 \tilde{\gamma}_{21}}, \quad \Delta \equiv \sqrt{\left(\tilde{\gamma}_{11}-\tilde{\gamma}_{22}\right)^{2}+4 \tilde{\gamma}_{12} \tilde{\gamma}_{21}}
$$

A special behaviour of eq. (3.21) is the UV-isolated constant solution $r(t)=r_{-}$, which acts as an IR-attractor. More generally, solutions are attracted in the UV towards the point $r(t)=r_{+}$. In any case, for $t \rightarrow \infty$, the ratio $r$ is always finite and thus all masses become typically comparable, despite experiencing a common overall infinite rescaling. Mass hierarchies do not arise dynamically in this context.

As an additional remark, note the behaviour of eq. (3.21). For $r_{0}>0$, the ratio $r$ is always non-vanishing, finite, and positive at all scales: there is no dynamical mechanism of spontaneous symmetry breaking. For $r_{-}<r_{0}<0$ (recall that $r_{-}$is always negative

\footnotetext{
${ }^{6}$ Note that eq. (3.20) is formally identical to the RGE for the ratio between gauge and quartic couplings, see eq. (2.20). An important difference is that, while eq. (2.20) is valid only for perturbative values of the coupling constants, eq. (3.20) is valid for any value of $r$.
} 
and $r_{+}$positive), the solution crosses $r=0$, where $m_{1}^{2}$ changes sign, and asymptotically flows towards $r_{+}$. For $r_{0}<r_{-}$, the solution crosses $1 / r=0$, where $m_{2}^{2}$ changes sign, before flowing to $r_{+}$.

Let us consider now the separate running of the mass parameters $m_{1,2}^{2}$ according to their RG evolution $d m_{i}^{2} / d t=\gamma_{i j} m_{j}^{2}$. In the asymptotic region where $\gamma_{i j}(t)=\tilde{\gamma}_{i j} / t$ holds, we can express the low-energy value of $m_{1}^{2}$ as

$$
\begin{aligned}
m_{1}^{2}\left(t_{0}\right) & =\frac{m_{1}^{2}(t)}{a_{+}\left(t / t_{0}\right)^{c_{+}}+a_{-}\left(t / t_{0}\right)^{c_{-}}}+\frac{b\left[\left(t / t_{0}\right)^{\Delta}-1\right]}{a_{+}+a_{-}\left(t / t_{0}\right)^{\Delta}} m_{2}^{2}\left(t_{0}\right) \\
a_{ \pm} & \equiv \frac{1}{2} \pm \frac{\tilde{\gamma}_{11}-\tilde{\gamma}_{22}}{2 \Delta}, \quad b \equiv \frac{\tilde{\gamma}_{12}}{\Delta}, \quad c_{ \pm} \equiv \frac{\tilde{\gamma}_{11}+\tilde{\gamma}_{22} \pm \Delta}{2}
\end{aligned}
$$

where $c_{ \pm}$are the two eigenvalues of the matrix $\tilde{\gamma}$ and $\Delta=c_{+}-c_{-}$is given in eq. (3.22).

The first term in the right-hand side of eq. (3.23) represents the boundary condition for $m_{1}^{2}$ in the far UV, once we take the limit $t \rightarrow \infty$. The interesting term is the second one, which measures how $m_{2}^{2}\left(t_{0}\right)$ affects $m_{1}^{2}\left(t_{0}\right)$ at the quantum level. In the case of a finite logarithmic running from the scale $\mu$ to some cut-off scale $\Lambda$, expanding eq. (3.23) we find

$$
\delta m_{1}^{2}(\mu) \approx m_{2}^{2}(\mu) \frac{\tilde{\gamma}_{12}}{(4 \pi)^{2}} \ln \frac{\Lambda^{2}}{\mu^{2}} .
$$

This corresponds to the familiar result that the hierarchy between two scalar masses $m_{1}^{2} \ll$ $m_{2}^{2}$ is destabilised by a one-loop correction $\delta m_{1}^{2}$ proportional to $m_{2}^{2}$ and to a logarithm, which becomes large whenever the theory can be extrapolated up to scales $\Lambda^{2} \gg m_{2}^{2}$. For instance, this is the source of the naturalness problem in supersymmetry, where the stop mass feeds into the Higgs mass at one-loop with a logarithm of the ratio between the weak and the GUT scale.

Naively, one could expect that, since in a TAF theory we effectively take $\Lambda \rightarrow \infty$, the coefficient measuring the contribution of $m_{2}^{2}$ to $\delta m_{1}^{2}$ must blow up to infinity, leading to a situation in which mass hierarchies are completely out of control. Equation (3.23) shows that this is not the case. As we send $t \rightarrow \infty$, the coefficient in front of $m_{2}^{2}\left(t_{0}\right)$ (which measures $\delta m_{1}^{2} / m_{2}^{2}$ at $\left.t_{0}\right)$ remains finite, no matter what the sign of $\Delta$ is. Moreover, note that this coefficient is given by a ratio between $b$ and $a_{ \pm}$: it is of order unity and it is no longer suppressed by the loop factor $(4 \pi)^{2}$, independently of the size of the coupling constants. The quantum correction $\delta m_{1}^{2}$ is parametrically equal to $m_{2}^{2}$, with no coupling constant or loop suppression, no matter how small is the coupling involved.

In conclusion, we have found that the infinite renormalisation of the mass parameters always leads to a finite result for the coefficient measuring how one scalar mass feeds into a smaller scalar mass. The infinite resummation eliminates the loop factor, insuring that all scalar masses at low energy be equal, within factors of order one. This has important consequences for the implementation of the naturalness criterion: in a TAF theory without special protection mechanisms (such as dynamical generation of masses at low scale [2-35]), any significant separation between mass scales entails a naturalness problem. 


\section{Asymptotic behaviour of the Standard Model}

As an illustrative example, we apply the method described in section 3 to the Standard Model, ignoring gravity. The coupling constants are the gauge couplings $g_{1}, g_{2}, g_{3}$, the Yukawa couplings $y_{t}, y_{b}, y_{\tau}, y_{\nu}$ (for simplicity we set to zero the Yukawa couplings of the first two generations), and the Higgs quartic coupling $\lambda$. Since we want to use the SM to elucidate our method, we will follow the analysis of section 3 step by step.

The approximation of ignoring interactions from the gravitational sector, or any other super-weak interactions from additional sectors, is justified by the assumptions of softened gravity. The feebleness of these interactions makes sure that they will never be able to cure the Landau poles of SM couplings. Of course, gravitational or super-weak interactions could affect the RG trajectories of SM couplings in the far UV, where the SM couplings asymptotically vanish, but cannot turn a non-TAF into a TAF theory. So our results, which ignore the effect of possible super-weak interactions, can be conservatively viewed as describing only necessary conditions for TAF.

\subsection{SM gauge couplings}

As is well known, the one-loop RGE for the SM gauge couplings are

$$
\frac{d g_{1}^{2}}{d t}=\frac{41}{10} g_{1}^{4}, \quad \frac{d g_{2}^{2}}{d t}=-\frac{19}{6} g_{2}^{4}, \quad \frac{d g_{3}^{2}}{d t}=-7 g_{3}^{4} .
$$

According to eqs. (3.8)-(3.9), we have 4 possible fixed-flows

\begin{tabular}{|l|cccc|}
\hline & $\tilde{g}_{1 \infty}^{2}$ & $\tilde{g}_{2 \infty}^{2}$ & $\tilde{g}_{3 \infty}^{2}$ & $M$-eigenvalues \\
\hline Solution 1 & 0 & $6 / 19$ & $1 / 7$ & +-- \\
Solution 2 & 0 & $6 / 19$ & 0 & +-+ \\
Solution 3 & 0 & 0 & $1 / 7$ & ++- \\
Solution 4 & 0 & 0 & 0 & +++ \\
\hline
\end{tabular}

The last column in eq. (4.2) shows the signs of the respective eigenvalues of the matrix $M\left(x_{\infty}\right)$ defined in eq. (3.7). We recall that a negative eigenvalue corresponds to a UV-attractor (IR-repulsor), while a positive eigenvalue corresponds to a UV-repulsor (IRattractor) and thus to an IR prediction.

Of course hypercharge is not asymptotically free, so all solutions are unphysical, since they require $g_{1}=0$. Still, it is interesting to pursue the study of the SM because it presents a non-trivial structure of possible Landau poles for the Yukawas and the Higgs quartic, providing a good illustration of our method, and also because $g_{1} \approx 0$ might be viewed as a rough approximation for the SM at low energy or for extensions of the SM where hypercharge is embedded in a non-abelian gauge group.

We proceed by focusing on the phenomenologically most relevant case of the fixed-flow corresponding to solution 1 in in eq. (4.2), with $\tilde{g}_{1 \infty}=0$, which is IR-attractive (UVrepulsive) giving one IR prediction, and $\tilde{g}_{1 \infty}, \tilde{g}_{2 \infty} \neq 0$, which are UV-attractive giving no extra predictions. 

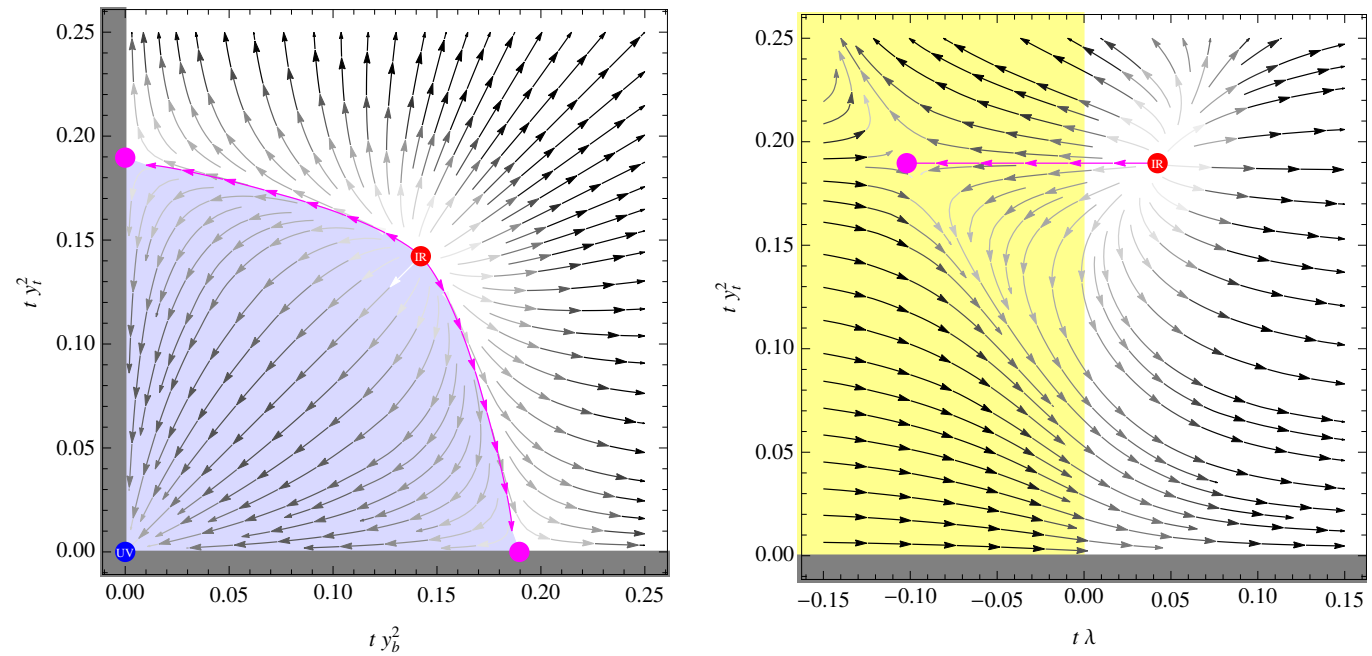

Figure 2. RG flows for the SM with $g_{1}=0$ in the plane of rescaled couplings $\tilde{y}^{2}=t y^{2}$ and $\tilde{\lambda}=t \lambda$. The dots are the fixed-flows of the rescaled couplings, with fully IR-attractive fixed points coloured red (2 combinations of couplings predicted), fully UV-attractors in blue, and hybrid in magenta (1 combination of couplings predicted). In the left plot we consider the top and bottom Yukawa couplings, setting to zero all other Yukawas. The basin of attraction of the UV fixed-flow is the shaded region; the basins of the hybrid fixed points are the magenta curves. In the right plot we consider the top Yukawa coupling and the Higgs quartic. No fully UV-attractive fixed-flow is present. The basic of attraction of the hybrid fixed-flow is the magenta curve.

\subsection{SM Yukawa couplings}

The one-loop RGE for the Yukawa couplings of the top quark $\left(y_{t}\right)$, the bottom $\left(y_{b}\right)$, the tau lepton $\left(y_{\tau}\right)$ and, if an interaction with a right-handed neutrino is present, of the neutrino $\left(y_{\nu}\right)$ are

$$
\begin{aligned}
& \frac{d y_{t}^{2}}{d t}=y_{t}^{2}\left(-\frac{17}{20} g_{1}^{2}-\frac{9}{4} g_{2}^{2}-8 g_{3}^{2}+\frac{9}{2} y_{t}^{2}+\frac{3}{2} y_{b}^{2}+y_{\tau}^{2}+y_{\nu}^{2}\right), \\
& \frac{d y_{b}^{2}}{d t}=y_{b}^{2}\left(-\frac{1}{4} g_{1}^{2}-\frac{9}{4} g_{2}^{2}-8 g_{3}^{2}+\frac{3}{2} y_{t}^{2}+\frac{9}{2} y_{b}^{2}+y_{\tau}^{2}+y_{\nu}^{2}\right) \\
& \frac{d y_{\tau}^{2}}{d t}=y_{\tau}^{2}\left(-\frac{9}{4} g_{1}^{2}-\frac{9}{4} g_{2}^{2}+3 y_{t}^{2}+3 y_{b}^{2}+\frac{5}{2} y_{\tau}^{2}-\frac{1}{2} y_{\nu}^{2}\right) \\
& \frac{d y_{\nu}^{2}}{d t}=y_{\nu}^{2}\left(-\frac{9}{20} g_{1}^{2}-\frac{9}{4} g_{2}^{2}+3 y_{t}^{2}+3 y_{b}^{2}-\frac{1}{2} y_{\tau}^{2}+\frac{5}{2} y_{\nu}^{2}\right) .
\end{aligned}
$$

For our choice of $\tilde{g}_{\infty}$, the system of equations that determines the Yukawa fixed-flows, given by eq. (3.11), has 4 possible solutions

\begin{tabular}{|l|ccccc|}
\hline & $\tilde{y}_{t \infty}^{2}$ & $\tilde{y}_{b \infty}^{2}$ & $\tilde{y}_{\tau \infty}^{2}$ & $\tilde{y}_{\nu \infty}^{2}$ & $M$-eigenvalues \\
\hline Solution 1 & $227 / 1197$ & 0 & 0 & 0 & +-++ \\
Solution 2 & 0 & $227 / 1197$ & 0 & 0 & -+++ \\
Solution 3 & $227 / 1596$ & $227 / 1596$ & 0 & 0 & ++++ \\
Solution 4 & 0 & 0 & 0 & 0 & --++ \\
\hline
\end{tabular}


The four fixed-flows and the flow restricted to the $\left(\tilde{y}_{t}, \tilde{y}_{b}\right)$ plane are plotted in figure 2a, where solutions 1 and 2 are plotted in magenta, point 3 (fully IR-attractive) in red, point 4 (UV-attractive) in blue. Solution 3 makes four predictions in the IR: $y_{t}\left(M_{t}\right)=y_{b}\left(M_{t}\right)=$ $0.879, y_{\tau}\left(M_{t}\right)=y_{\nu}\left(M_{t}\right)=0$. This corresponds to the pole masses $M_{t}=M_{b} \approx 163 \mathrm{GeV}$ and $M_{\tau}=M_{\nu}=0$. Solution 2 makes three predictions in the IR, giving $M_{b} \simeq 186 \mathrm{GeV}$ and $M_{\tau}=M_{\nu}=0$. Neither case gives a reasonable result for the bottom-quark mass.

Solution 4 corresponds to vanishing $\tilde{y}_{\infty}$ for all Yukawa couplings. However, there is an important difference between quark and lepton Yukawa couplings. Since $\tilde{y}_{\tau, \nu \infty}=0$ are UV-repulsive, Landau poles can be avoided only if the lepton Yukawa couplings are exactly zero in the IR. On the other hand, since $\tilde{y}_{t, b_{\infty}}=0$ are UV-attractive, the quark Yukawa couplings satisfy TAF for a range of IR boundary conditions. Indeed, near the fixed-flow of solution 4 and for small Yukawas, eqs. (4.3)-(4.4) become

$$
\frac{d y_{t, b}^{2}}{d t} \simeq-c \frac{y_{t, b}^{2}}{t}, \quad c=\frac{493}{266}
$$

The solutions are $y_{t, b}^{2} \propto t^{-c}$, which in the asymptotic region $t \rightarrow \infty$ become negligible with respect to $g_{2,3}^{2} \sim 1 / t$, since $c>1$. Although solution 4 seems phenomenologically plausible as far as Yukawas are concerned, it is not compatible with TAF for the Higgs quartic, as we will show in the next section. For this reason, in the following we will focus on solution 1.

Solution 1 gives three predictions: zero $\tau$ and neutrino masses, and top mass at its IR fixed-flow. The bottom Yukawa is near a UV-attractive fixed-flow, hence the bottom-quark mass is not determined. This can be seen explicitly by writing eq. (4.4) near the fixed-flow of solution 1 , for small $y_{b, \tau, \nu} \ll 1$

$$
\frac{d y_{b}^{2}}{d t} \simeq-c \frac{y_{b}^{2}}{t}, \quad c=\frac{626}{399}
$$

The solution, $y_{b}^{2} \propto t^{-c}$ goes to zero faster than the gauge couplings for $t \rightarrow \infty$, since $c>1$.

\subsection{SM Higgs quartic coupling}

The one-loop RGE for the Higgs quartic coupling that parameterises the potential $\lambda|H|^{4}$ is

$$
\begin{aligned}
\frac{d \lambda}{d t}= & 12 \lambda^{2}+\lambda\left(6 y_{t}^{2}+6 y_{b}^{2}+2 y_{\tau}^{2}+2 y_{\nu}^{2}-\frac{9}{2} g_{2}^{2}-\frac{9}{10} g_{1}^{2}\right) \\
& -3 y_{t}^{4}-3 y_{b}^{4}-y_{\tau}^{4}-y_{\nu}^{4}+\frac{9}{16} g_{2}^{4}+\frac{27}{400} g_{1}^{4}+\frac{9}{40} g_{2}^{2} g_{1}^{2} .
\end{aligned}
$$

The fixed-flows are obtained by solving eq. (3.15). As anticipated, we find no solutions for $\tilde{\lambda}_{\infty}$ when all Yukawa couplings are on their vanishing fixed-flow $\tilde{y}=0$, i.e. solution 4 in eq. (4.7). On the other hand, we find two solutions for $\tilde{\lambda}_{\infty}$ in each of the other three cases, i.e. solutions 1-3 in eq. (4.7). This is an example of how Yukawa couplings sitting on some non-vanishing fixed-flow can save the running of a scalar quartic coupling, otherwise doomed to suffer from Landau poles, and produce asymptotically-free solutions. Among the three possible cases, we concentrate on solution 1 in eq. (4.7) for the Yukawas, which 
is the most propitious from a phenomenological point of view. Then, eq. (3.15) admits the following two solutions for $\tilde{\lambda}_{\infty}$ :

\begin{tabular}{|l|ccc|}
\hline & $\tilde{\lambda}_{\infty}$ & $M$-eigenvalue & potential \\
\hline Solution 1 & $\frac{-143+\sqrt{119402}}{4788} \approx+0.0423$ & + & stable \\
Solution 2 & $\frac{-143-\sqrt{119402}}{4788} \approx-0.1020$ & - & unstable \\
\hline
\end{tabular}

figure $2 \mathrm{~b}$ shows the RG flow in the plane $\tilde{\lambda}-\tilde{y}_{t}$. We find three possible behaviours. (i) A generic point in the plane flows towards a Landau pole of either $\lambda$ or $y_{t}$. (ii) If we select $\tilde{y}_{t}=\tilde{y}_{t \infty}$, then $\tilde{\lambda}$ flows towards the UV-attractive fixed-flow, where $\lambda$ is negative, making the EW vacuum potentially unstable. In this case, one parameter is predicted in the IR (the top-quark mass) and the dimensionality of the basin of attraction is reduced by one. (iii) The solution sits on the IR-attractor, for which the basin of attraction is reduced by two and both the top-quark and the Higgs masses are predicted. The prediction of the IR-attractive solution 1 in eq. (4.11) corresponds to $\lambda\left(M_{t}\right)=0.217$, i.e. to a pole Higgs mass $M_{h}=163 \mathrm{GeV}$.

The negative value of $\lambda$ at the UV-attractive solution 2 in eq. (4.11) means that the EW vacuum is unstable. However, this situation is not necessarily ruled out if the tunnelling rate is slower than the age of the universe. Considering a field direction along which the quantum-corrected potential is $V \approx \frac{1}{4} \lambda(\mu \approx h) h^{4}$, the EW vacuum is sufficiently long-lived provided that $\lambda$ does not become too much negative at large energy. The Fubini bounce solution to the classical field equation, $h(r)=\sqrt{-2 / \lambda} \times 2 R /\left(r^{2}+R^{2}\right)$, has tree-level action $S=8 \pi^{2} / 3|\lambda|$ where $R$ is a free parameter [59-62]. Thereby, imposing a negligible probability for the vacuum to have decayed during its past history

$$
e^{-S} \ll\left(R H_{0}\right)^{4}
$$

where $H_{0}$ is the present Hubble constant, we obtain

$$
\lambda\left(\mu \sim \frac{1}{R}\right)>\frac{2 \pi^{2}}{3 \ln H_{0} R} \stackrel{t \rightarrow \infty}{\simeq}-\frac{1}{12 t} .
$$

The fixed-flow in solution 2 of eq. (4.11) corresponds to an asymptotic behaviour $\lambda \approx$ $-1 /(9.8 t)$, which slightly violates the metastability constraint in eq. (4.13).

In conclusion, TAF imposes strong constraints on the Higgs quartic coupling: either $M_{h}=163 \mathrm{GeV}$, or $M_{h}<163 \mathrm{GeV}$ and the lifetime of the $\mathrm{EW}$ vacuum is shorter than the age of the universe. Neither possibility is realistic.

\subsection{RG flow of the SM couplings}

For physical values of its coupling constants, the SM is not an asymptotically free theory. We have found that the closest approximation to physical reality for the SM to be a TAF 


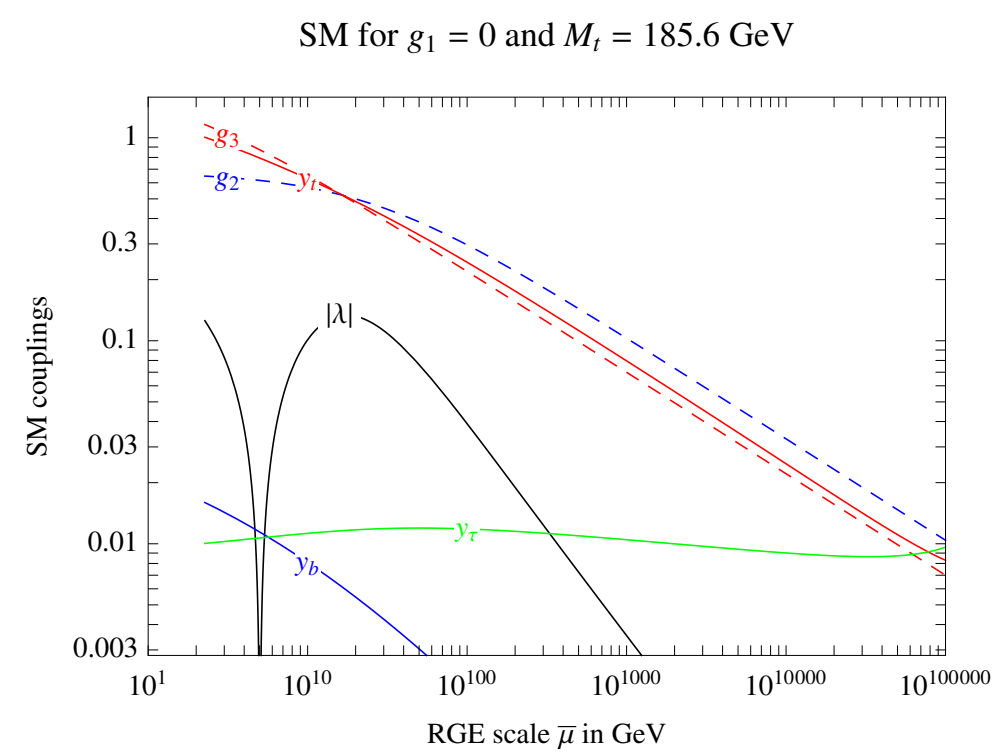

Figure 3. RG running towards infinite energy (note the double-log scale for the $\overline{\mathrm{MS}}$ renormalisation scale $\bar{\mu}$ ) in the SM for the measured values of $M_{h}, M_{b}, M_{\tau}, g_{2}, g_{3}$ and for the values of $g_{1}$ and $M_{t}$ needed to achieve TAF: $g_{1}=0$ and $M_{t}=185.6 \mathrm{GeV}$.

theory is that its coupling constants lie in the basin of attraction of the following fixed-flow

\begin{tabular}{|cccccccc|}
\hline$\tilde{g}_{1 \infty}^{2}$ & $\tilde{g}_{2 \infty}^{2}$ & $\tilde{g}_{3 \infty}^{2}$ & $\tilde{y}_{t \infty}^{2}$ & $\tilde{y}_{b \infty}^{2}$ & $\tilde{y}_{\tau \infty}^{2}$ & $\tilde{y}_{\nu \infty}^{2}$ & $\tilde{\lambda}_{\infty}$ \\
\hline 0 & $\frac{6}{19}$ & $\frac{1}{7}$ & $\frac{227}{1197}$ & 0 & 0 & 0 & $\frac{-143+\sqrt{119402}}{4788}$ \\
+ & - & - & + & - & + & + & + \\
\hline
\end{tabular}

The matrix $M\left(x_{\infty}\right)$ has 5 positive eigenvalues; hence, within the 8-dimensional space of couplings $x=\left\{\tilde{g}_{1,2,3}, \tilde{y}_{t, b, \tau, \nu}, \tilde{\lambda}\right\}$, the basin of attraction of the fixed-flow in eq. (4.14) has dimensionality equal to $8-5=3$. As a result, TAF makes the following 5 predictions on physical parameters. The hypercharge gauge coupling must be zero; the tau lepton and neutrino must be massless; the top quark mass must be $M_{t}=186 \mathrm{GeV}$, which is $7 \%$ higher than the observed value; the Higgs mass must be $M_{h}=163 \mathrm{GeV}$, which is $30 \%$ higher than the observed value (or $M_{h}<163 \mathrm{GeV}$, but the $\mathrm{EW}$ vacuum is unstable). None of these predictions is correct, but they are not bad approximations of reality. It is conceivable that these wrong predictions can be cured in extensions of the SM where hypercharge is embedded in a non-abelian group and where we expect corrections at least of order $g_{1}^{2} / g_{2}^{2}$.

In figure 3 we show the RG flow of the SM coupling constants, taking $g_{1}$ and $y_{t}$ at their fixed-flows, while all other parameters are equal to their physical values in the IR. The figure is obtained by solving the SM RGE in the 3-loop approximation. For the physical value of the $\tau$ mass, we observe that $y_{\tau}$ starts small in the IR, but becomes the largest coupling at $10^{10^{5}} \mathrm{GeV}$, where soon reaches a Landau pole. For the physical value of the Higgs mass, the coupling $\lambda$ becomes negative at an intermediate scale, before flowing to zero in the deep UV, barring the effect of the Landau pole in the $\tau$ Yukawa. The coupling $\lambda$ can be asymptotically free and always positive only for the special IR condition 
$M_{h}=163 \mathrm{GeV}$ (with $M_{t}=186 \mathrm{GeV}$ ). This condition on $M_{h}$ and $M_{t}$ corresponds to the Pendleton-Ross point [57] or to the tip of the SM phase diagram (shown in $[63,64]$ ), although the numerical values of $M_{h}$ and $M_{t}$ quoted here are somehow different, since in our calculation we set $g_{1}=0$.

\section{TAF extensions of the SM}

\subsection{Grand unification}

We start our exploration of extensions of the SM by constructing asymptotically-free versions of grand-unified theories (GUT). These examples bear no relevance on the issue of naturalness in theories with softened gravity because they introduce in the observable sector a mass scale $M_{\mathrm{GUT}}$ much larger the weak scale, which feeds unnaturally large corrections to the Higgs mass $\delta M_{h}^{2} \sim g_{\text {GUT }}^{2} M_{\text {GUT }}^{2} /(4 \pi)^{2}$. Nevertheless, we find the study instructive for its own sake and we present it here.

The simplest GUT is based on the gauge group SU(5), with 3 generations of chiral fermions in the $\overline{5} \oplus 10$ representation of SU(5), and scalars $H$ and $\Sigma$ in the $5 \oplus 24$ representation. This theory does not satisfy the TAF requirements. However, independently of TAF considerations, the theory cannot be considered realistic, since it gives some wrong predictions for quark and lepton masses.

We then consider an extension of the minimal SU(5) setup that simultaneously satisfies the conditions of TAF and of an acceptable fermion mass spectrum. We add 3 generations of vector-like fermions $\psi_{5}, \psi_{5}$ and $\psi_{24}$ in the $5 \oplus \overline{5} \oplus 24$ representation of SU(5) with mass terms $M_{5} \psi_{5} \psi_{5}+M_{24} \psi_{24}^{2} / 2$. The Yukawa couplings, written for simplicity only for the third generation, are

$$
\begin{aligned}
\mathscr{L}_{Y}= & -\frac{y_{t}}{8} 1010 H+\left(y_{b} \overline{5}+y_{b}^{\prime} \psi_{\overline{5}}\right) 10 H^{*}+\left(y_{\nu} \overline{5}+y_{\nu}^{\prime} \psi_{\overline{5}}\right) \psi_{24} H \\
& +y_{\nu}^{\prime \prime} \psi_{5} \psi_{24} H^{*}+\left(y_{m} \overline{5}+y_{m}^{\prime} \psi_{\overline{5}}\right) \Sigma \psi_{5}+y_{\lambda} \operatorname{Tr}\left(\Sigma \psi_{24}^{2}\right) .
\end{aligned}
$$

The coupling $y_{t}$ gives the top-quark Yukawa and $y_{b}$ gives the bottom-quark and $\tau$-lepton Yukawas. The coupling $y_{m}$ induces an SU(5)-breaking mixing between $\overline{5}$ and $\psi_{\overline{5}}$, which modifies the phenomenologically wrong equality between down-quark and the chargedlepton Yukawa couplings predicted by minimal SU(5). The split multiplets contribute to threshold corrections to the SU(5) prediction for gauge coupling unification, which could make the result compatible with low-energy measurements. The coupling $y_{\nu}$ generates a neutrino Yukawa, in which the right-handed neutrino is identified as the SM singlet in $\psi_{24}$. The simpler alternative of generating the neutrino Yukawa coupling with a right-handed neutrino as a singlet of SU(5) does not satisfy the TAF condition of eq. (2.11), unless the $\beta$-function of $g_{5}$ is reduced by adding extra matter. A two-generation SU(5) TAF model that ignores this issue was presented in [53-55].

The most general quartic potential is

$$
V_{4}=\lambda_{H}|H|^{4}+\lambda_{\Sigma} \operatorname{Tr}\left(\Sigma^{4}\right)+\lambda_{\Sigma}^{\prime} \operatorname{Tr}\left(\Sigma^{2}\right)^{2}+\lambda_{H \Sigma} H^{\dagger} \Sigma^{2} H+\lambda_{H \Sigma}^{\prime}|H|^{2} \operatorname{Tr}\left(\Sigma^{2}\right) .
$$


The purely quartic potential, restricted to $\Sigma$, is positive definite when ${ }^{7}$

$$
\lambda_{\Sigma}^{\prime}>-\frac{7}{30} \lambda_{\Sigma} \quad \text { for } \lambda_{\Sigma}>0 \quad \text { and } \quad \lambda_{\Sigma}^{\prime}>-\frac{13}{20} \lambda_{\Sigma} \text { for } \lambda_{\Sigma}<0 .
$$

The violation of the first condition leads to the symmetry breaking pattern $\mathrm{SU}(5) \rightarrow G_{\mathrm{SM}}$ à la Coleman-Weinberg; the violation of the second condition leads to $\mathrm{SU}(5) \rightarrow \mathrm{SU}(4) \otimes \mathrm{U}(1)$.

The $\beta$-function coefficient for the unified gauge coupling is $b_{5}=4 / 3$. For the Yukawa couplings, we find various fixed-flow solutions and the phenomenologically most interesting case is

\begin{tabular}{|c|cccccccccc|}
\hline & $\tilde{g}_{5 \infty}^{2}$ & $\tilde{y}_{t \infty}^{2}$ & $\tilde{y}_{b \infty}^{2}$ & $\tilde{y}_{b \infty}^{\prime 2}$ & $\tilde{y}_{\nu \infty}^{2}$ & $\tilde{y}_{\nu \infty}^{\prime 2}$ & $\tilde{y}_{\nu \infty}^{\prime \prime 2}$ & $\tilde{y}_{m \infty}^{2}$ & $\tilde{y}_{m \infty}^{\prime 2}$ & $\tilde{y}_{\lambda \infty}^{2}$ \\
\hline Fixed-flow & $3 / 4$ & $38 / 15$ & 0 & 0 & 0 & 0 & 0 & $49 / 16$ & 0 & 0 \\
$M$-Eigenvalues & - & + & - & - & - & - & - & + & 0 & - \\
\hline
\end{tabular}

The lower row in eq. (5.4) shows the signs of the eigenvalues of the $M$ matrix: only two combinations of Yukawa couplings are predicted. The vanishing eigenvalue arises because the couplings are accidentally invariant under global $\mathrm{SU}(2)$ rotations acting on $\left(\overline{5}, \psi_{\overline{5}}\right)$. All other Yukawa couplings with $\tilde{y}_{\infty}^{2}=0$ have negative eigenvalues and therefore their IR values are non-vanishing, but cannot be predicted by the TAF requirement.

One predicted combination of Yukawa couplings involves the top coupling $y_{t}$. Indeed its RGE is

$$
\frac{d y_{t}^{2}}{d t}=y_{t}^{2}\left(-f_{g} g_{5}^{2}+f_{y} y_{t}^{2}-2 y_{b}^{2}+\frac{12}{5} y_{\nu}^{2}\right) \quad \text { with } \quad f_{g}=\frac{108}{5}, \quad f_{y}=6 .
$$

Thereby, for $y_{b}, y_{\nu} \ll y_{t}$ one has the prediction $y_{t}^{2} / g_{5}^{2} \simeq\left(f_{g}-b_{5}\right) / f_{y}$. However, there is no unique way of relating this prediction to the physical value of the top mass, because other Yukawa couplings can affect the IR value of $y_{t}$.

The solution for the Yukawa couplings in eq. (5.4) allows for 4 fixed-flows for the quartics

\begin{tabular}{|l|ccccccc|}
\hline & $\tilde{\lambda}_{H \infty}$ & $\tilde{\lambda}_{\Sigma \infty}$ & $\tilde{\lambda}_{\Sigma^{\prime} \infty}^{\prime}$ & $\tilde{\lambda}_{H \Sigma \infty}$ & $\tilde{\lambda}_{H \Sigma \infty}^{\prime}$ & $M$-Eigenvalues & Potential \\
\hline Solution 1 & -1.16 & -0.326 & 0.185 & 0.610 & -0.003 & ----- & unstable \\
Solution 2 & -1.15 & -0.422 & 0.541 & 0.725 & 0.116 & ----+ & unstable \\
Solution 3 & 0.831 & -0.315 & 0.215 & 0.989 & -0.562 & ---++ & unstable \\
Solution 4 & 0.821 & -0.334 & 0.500 & 1.617 & -0.597 & --+++ & stable \\
\hline
\end{tabular}

The potential is stable only in the case of solution 4 , for which three combinations of quartic couplings are predicted in the IR by demanding that TAF is achieved.

In conclusion, it is not difficult to construct realistic TAF GUT models. The TAF conditions significantly constrain the field content of the theory, although any phenomenological prediction of low-energy parameters is highly model-dependent.

\footnotetext{
${ }^{7}$ We used the identity $7 / 30 \leq \operatorname{Tr}\left(\Sigma^{4}\right) / \operatorname{Tr}^{2}\left(\Sigma^{2}\right) \leq 13 / 20$. The stability conditions for the full potential $V_{4}$ are much more complicated [65-67].
} 


\subsection{TAF extensions of the SM at the weak scale}

As discussed in section 4, the SM does not satisfy TAF. For theories with softened gravity to respect naturalness, the SM must be modified at the weak scale and made compatible with TAF. So we address now the question of how to construct such modifications. The first problem is that hypercharge is not asymptotically free. This implies that, in any TAF extension of the SM, one must embed hypercharge in a non-abelian gauge group. Such extensions have the additional advantage of explaining the observed quantisation of electric charge.

The hypercharges $Y$ of SM fermions satisfy the relation $Y=T_{3 R}+(B-L) / 2$, where $T_{3 R}$ is the third component of the right-handed isospin $\mathrm{SU}(2)_{R}$. Thus, the most straightforward possibility is to promote $T_{3 R}$ to the full non-abelian $\mathrm{SU}(2)_{R}$ gauge group, with SM field assignment as in table 2. Similarly, $\mathrm{U}(1)_{B-L}$ is not asymptotically free, so one needs to embed it into a non-abelian group. Given the known values of the $B-L$ charges, we find only two possibilities that do not lead to proton decay:

- Merging $B-L$ with $\mathrm{SU}(3)_{c}$ into the Pati-Salam $\mathrm{SU}(4)_{\mathrm{PS}}$, such that the full gauge group is

$$
G_{224}=\mathrm{SU}(2)_{L} \otimes \mathrm{SU}(2)_{R} \otimes \mathrm{SU}(4)_{\mathrm{PS}} .
$$

$B-L$ arises as the diagonal $\operatorname{diag}(1,1,1,-3) / \sqrt{24}$ generator of $\mathrm{SU}(4)_{\mathrm{PS}}$.

- Merging $B-L$ with $\mathrm{SU}(2)_{L} \otimes \mathrm{SU}(2)_{R}$ into $\mathrm{SU}(3)_{L} \otimes \mathrm{SU}(3)_{R}$, such that the full gauge group is

$$
G_{333}=\mathrm{SU}(3)_{L} \otimes \mathrm{SU}(3)_{R} \otimes \mathrm{SU}(3)_{c} .
$$

$B-L$ arises as the combination of the diagonal $\operatorname{diag}(1,1,-2) / \sqrt{12}$ generators of $\mathrm{SU}(3)_{L}$ and of $\mathrm{SU}(3)_{R}$.

Before presenting specific models, in the rest of this section we assess some generic common features. First, we discuss in section 5.3 the flavour-violations coming from the two-Higgs doublet structure implied by the left-right symmetry. Then, in section 5.4 we study the phenomenological constraints on the existence of the charged and neutral gauge bosons of $\mathrm{SU}(2)_{R}$.

\subsection{New heavy Higgs and flavour processes}

Both options we are considering for embedding the SM into a non-abelian group (PatiSalam and trinification) include $\mathrm{SU}(2)_{R}$, and thus the SM Higgs doublet must be extended at least into the structure

$$
\phi=\left(\begin{array}{ll}
H_{U}^{0} & H_{D}^{+} \\
H_{U}^{-} & H_{D}^{0}
\end{array}\right)=\left(\begin{array}{ll}
H_{U} & H_{D}
\end{array}\right),
$$

which transforms as $\left(2_{L}, \overline{2}_{R}\right)$ under $\mathrm{SU}(2)_{L} \times \mathrm{SU}(2)_{R}$. The field $\phi$ contains two Higgs doublets, transforming under the $\mathrm{SM} \mathrm{SU}(2)_{L} \times \mathrm{U}(1)_{Y}$ as $H_{U} \sim(2,-1 / 2)$ and $H_{D} \sim$ $(2,1 / 2)$. The two-Higgs structure is often troublesome because it generates scalar-mediated 
flavour-changing neutral-current (FCNC) interactions. We show here how the problem can be avoided with an appropriate flavour structure of quark Yukawa couplings.

Denoting as $q_{L}=\left(u_{L}, d_{L}\right)$ and $q_{R}=\left(u_{R}, d_{R}\right)$ the SM quarks which transform as $\left(2_{L}, 1_{R}\right)$ and $\left(1_{L}, 2_{R}\right)$, the $\mathrm{SU}(2)_{L} \times \mathrm{SU}(2)_{R}$ invariant quark-Yukawa interactions are

$$
-\mathscr{L}_{Y}^{q}=\bar{q}_{L}\left(Y \phi+Y_{c} \phi^{c}\right) q_{R}+\text { h.c. }=\bar{q}_{L}\left(Y H_{U}+Y_{c} H_{D}^{c}\right) u_{R}+\bar{q}_{L}\left(Y H_{D}-Y_{c} H_{U}^{c}\right) d_{R}+\text { h.c. }
$$

where

$$
\phi^{c} \equiv \epsilon^{T} \phi^{*} \epsilon=\left(\begin{array}{cc}
H_{D}^{0 *} & -H_{U}^{+} \\
-H_{D}^{-} & H_{U}^{0 *}
\end{array}\right)=\left(\begin{array}{ll}
H_{D}^{c} & -H_{U}^{c}
\end{array}\right),
$$

and $H_{U, D}^{c}=\epsilon H_{U, D}^{*}, \epsilon=i \sigma_{2}$, and $Y$ and $Y_{c}$ are two different Yukawa matrices in flavour space. It is convenient to rewrite the two doublets $H_{U}$ and $H_{D}$ in terms of a SM-like doublet $h$ and of a heavy doublet $H$, defined such that $\langle H\rangle=(0,0)$ and $\langle h\rangle=(0, v)$ with $v=\left(v_{d}^{2}+v_{u}^{2}\right)^{1 / 2}$ :

$$
\left(\begin{array}{c}
h \\
H
\end{array}\right)=\left(\begin{array}{cc}
\cos \beta & \sin \beta \\
-\sin \beta & \cos \beta
\end{array}\right)\left(\begin{array}{c}
H_{D} \\
-H_{U}^{c}
\end{array}\right), \quad \sin \beta=\frac{v_{u}}{v}, \quad \cos \beta=\frac{v_{d}}{v} .
$$

Defining the SM Yukawa couplings $Y_{D}$ and $Y_{U}$ as

$$
\left(\begin{array}{l}
Y_{D} \\
Y_{U}
\end{array}\right)=\left(\begin{array}{cc}
\cos \beta & \sin \beta \\
\sin \beta & \cos \beta
\end{array}\right)\left(\begin{array}{c}
Y \\
Y_{c}
\end{array}\right)
$$

the Lagrangian in eq. (5.10) becomes

$$
\begin{aligned}
&-\mathscr{L}_{Y}^{q}=\bar{q}_{L} Y_{D} d_{R} h+\bar{q}_{L}\left(\frac{Y_{U}}{\cos 2 \beta}-\tan 2 \beta Y_{D}\right) d_{R} H \\
&+\bar{q}_{L} Y_{U} u_{R} h^{c}+\bar{q}_{L}\left(\frac{Y_{D}}{\cos 2 \beta}-\tan 2 \beta Y_{U}\right) u_{R} H^{c} .
\end{aligned}
$$

We see that down-type $H$-mediated FCNCs are controlled by the off-diagonal entries of $Y_{U}$, in the basis where $Y_{D}$ is diagonal, and viceversa. In the $\mathrm{SU}(2)_{L} \times \mathrm{SU}(2)_{R}$ invariant basis where $Y_{D}$ is diagonal we can write

$$
Y_{U}^{\mathrm{d}-\text { base }}=V^{\dagger} \lambda_{u} V_{R}, \quad Y_{D}^{\mathrm{d}-\text { base }}=\lambda_{d},
$$

where $\lambda_{u, d}$ are diagonal matrices of the quark masses, $V$ is the usual CKM matrix, and $V_{R}$ is a new unitary matrix which controls all new flavour violations.

The effects of $V_{R}$ are best understood by considering the accidental global flavour symmetry of the model: $\mathrm{U}(3)_{L} \times \mathrm{U}(3)_{R}$ explicitly broken by $Y_{U}$ and $Y_{D}$ which both transform as $(3, \overline{3})$. From the SM point of view, such flavour structure is equivalent to $\mathrm{U}(3)_{q_{L}} \times \mathrm{U}(3)_{u_{R}} \times \mathrm{U}(3)_{d_{R}}$, with three independent spurions: $Y_{U} \sim(3, \overline{3}, 1)$ and $Y_{D} \sim$ $(3,1, \overline{3})$, as in Minimal Flavour Violation (MFV) [68], plus $V_{R} \sim(1,3, \overline{3})$ [69]. 
In the quark mass eigenbasis, the interactions of the neutral Higgs $h^{0}$ and $H^{0}$, derived from eq. (5.14) are

$$
\begin{aligned}
&-\mathscr{L}_{Y}^{q}=\bar{d}_{L} \lambda_{d} d_{R} h^{0}+\bar{d}_{L}\left(\frac{V^{\dagger} \lambda_{u} V_{R}}{\cos 2 \beta}-\tan 2 \beta \lambda_{d}\right) d_{R} H^{0} \\
&+\bar{u}_{L} \lambda_{u} u_{R} h^{0 *}+\bar{u}_{L}\left(\frac{V \lambda_{d} V_{R}^{\dagger}}{\cos 2 \beta}-\tan 2 \beta \lambda_{u}\right) u_{R} H^{0 *} .
\end{aligned}
$$

Again, we observe that the flavour violations mediated at tree level by the neutral scalar $H^{0}$ are proportional to $\lambda_{u}$ in the down sector and to $\lambda_{d}$ in the up sector, and are expressed in terms of the CKM matrix $V$ and the new rotation matrix $V_{R}$.

Right-handed flavour mixing can be naturally small. On general grounds, $V_{R}$ can be parameterised as follows

$$
V_{R}=P_{u} \widetilde{V}_{R} P_{d}^{\dagger}, \quad P_{q}=\operatorname{diag}\left(e^{\phi_{1}^{q}}, e^{\phi_{2}^{q}}, e^{\phi_{3}^{q}}\right),
$$

where $\widetilde{V}_{R}$ is a CKM-like matrix, with 3 rotational angles and one phase, and $P_{u}$ and $P_{d}$ are diagonal phase matrices. One overall phase in $P_{u, d}$ is unphysical, while, in general, the other 5 are physical. ${ }^{8}$

Assuming a simple and natural form of Yukawa couplings - quasi-diagonal Yukawa matrices with small or negligible off-diagonal entries and no relations between them - the presence of left-handed CKM mixing implies a minimal amount of right-handed mixing given by

$$
\left\{\begin{array}{l}
\left|\left(V_{R}\right)_{u s}\right| \approx\left|V_{u s}\right| m_{d} / m_{s} \approx 10^{-2}, \\
\left|\left(V_{R}\right)_{c b}\right| \approx\left|V_{c b}\right| m_{s} / m_{b} \approx 10^{-3}, \\
\left|\left(V_{R}\right)_{u b}\right| \approx\left|V_{u b}\right| m_{d} / m_{b} \approx 10^{-5} .
\end{array}\right.
$$

Furthermore, this small amount of right-handed mixing is radiatively stable and is generated by RG corrections (more generally $\widetilde{V}_{R} \approx$ permutation matrix is radiatively stable too). This can be seen by observing that $\widetilde{V}_{R}=1$ implies $\left[Y_{U}^{\dagger} Y_{U}, Y_{D}^{\dagger} Y_{D}\right]=0$. As a result, entires proportional to $Y_{U}^{\dagger} Y_{U}$ and $Y_{D}^{\dagger} Y_{D}$ in the RG evolution of both $Y_{U}^{\dagger} Y_{U}$ and $Y_{D}^{\dagger} Y_{D}$ do not generate off-diagonal entries, if starting from an initial condition with $\widetilde{V}_{R}=11$. Off-diagonal terms in the right-handed sector are generated only from the contribution of mixed terms, $Y_{D}^{\dagger} Y_{U}$ and $Y_{U}^{\dagger} Y_{D}$, in the RG evolution of $Y_{U}^{\dagger} Y_{U}$ and $Y_{D}^{\dagger} Y_{D}$. The latter give rises to small deviations from $\widetilde{V}_{R}=1$, again suppressed by both off-diagonal CKM entires and small quark mass ratios: $\left|\left(\widetilde{V}_{R}\right)_{i>j}\right| \lesssim\left(m_{j} / m_{i}\right)\left|V_{i j}\right|$. A similar argument holds for the CP-violating phases.

Flavour bounds on heavy Higgs bosons. In order to evaluate the strength of FCNCs, we assume that $\widetilde{V}_{R}$ is about equal to the identity matrix,

$$
\widetilde{V}_{R}=\left(\begin{array}{ccc}
1 & s_{12} & s_{13} e^{-i \phi} \\
-s_{12} & 1 & s_{23} \\
-s_{13} e^{i \phi} & -s_{23} & 1
\end{array}\right)+\mathcal{O}\left(s_{i j} s_{k l}\right) .
$$

\footnotetext{
${ }^{8}$ These phases could be moved into the eigenvalues of $\lambda_{u, d}$. However, to avoid confusion, we work in the usual basis where such eigenvalues are real and positive and leave explicitly the phases in $P_{u, d}$.
} 


\begin{tabular}{|c|c|l|}
\hline system & effective operator & bound from $\Delta M_{\text {meson }}$ \\
\hline$B_{d}^{0}-\bar{B}_{d}^{0}$ & $\left(\bar{b}_{L} d_{R}\right)\left(\bar{b}_{R} d_{L}\right)$ & $\left|3.6 \times 10^{-8}+s_{13}\right|<1.7 \times 10^{-4}\left(M_{H} / 3 \mathrm{TeV}\right)^{2}$ \\
$B_{s}^{0}-\bar{B}_{s}^{0}$ & $\left(\bar{b}_{L} s_{R}\right)\left(\bar{b}_{R} s_{L}\right)$ & $\left|1.5 \times 10^{-4}-s_{23}\right|<1.0 \times 10^{-3}\left(M_{H} / 3 \mathrm{TeV}\right)^{2}$ \\
$K^{0}-\bar{K}^{0}$ & $\left(\bar{s}_{L} d_{R}\right)\left(\bar{s}_{R} d_{L}\right)$ & $\left|6.2 \times 10^{-4}-s_{12}+11 s_{13}\right|<1.0 \times 10^{-2}\left(M_{H} / 3 \mathrm{TeV}\right)^{2}$ \\
$D^{0}-\bar{D}^{0}$ & $\left(\bar{c}_{L} u_{R}\right)\left(\bar{c}_{R} u_{L}\right)$ & $\left|1.1 \times 10^{-2}-s_{12}-2.0 s_{13}\right|<9.5\left(M_{H} / 3 \mathrm{TeV}\right)^{2}$ \\
\hline
\end{tabular}

Table 1. Constraints on right-handed mixing angles $\left(s_{12}, s_{13}, s_{23}\right)$ as functions of the heavy Higgs mass $\left(M_{H}\right)$ from $\Delta F=2$ processes, assuming $\tan \beta \gg 1$. For generic values of $\tan \beta$, the constraints are obtained with the replacement $M_{H} \rightarrow M_{H}|\cos 2 \beta|$.

In analogy with the CKM matrix, and motivated by the need to satisfy the strong constraints on FCNC (see below), we assume $\left|s_{13}\right|<\left|s_{23}\right|<\left|s_{12}\right| \ll 1$.

Integrating out the heavy Higgs $H^{0}$ (assuming a negligible $h-H$ mixing), the interactions in eq. (5.16) give the following $\Delta F=2$ dimension-six effective operators

$$
\begin{aligned}
& \mathcal{L}_{\Delta F=2}=\frac{X_{i j}^{(d)}}{M_{H}^{2}}\left(\bar{d}_{L}^{i} d_{R}^{j}\right)\left(\bar{d}_{R}^{i} d_{L}^{j}\right), \quad X_{i j}^{(d)}=\frac{1}{\cos ^{2} 2 \beta}\left(\sum_{k} \lambda_{u_{k}} V_{k i}^{*} V_{R_{k j}}\right)\left(\sum_{\ell} \lambda_{u_{\ell}} V_{\ell j} V_{R_{\ell i}}^{*}\right), \\
& \mathcal{L}_{\Delta F=2}=\frac{X_{i j}^{(u)}}{M_{H}^{2}}\left(\bar{u}_{L}^{i} u_{R}^{j}\right)\left(\bar{u}_{R}^{i} u_{L}^{j}\right), \quad X_{i j}^{(u)}=\frac{1}{\cos ^{2} 2 \beta}\left(\sum_{k} \lambda_{d_{k}} V_{i k} V_{R_{j k}}^{*}\right)\left(\sum_{\ell} \lambda_{d_{\ell}} V_{j \ell}^{*} V_{R_{i \ell}}\right) .
\end{aligned}
$$

The $\tan \beta$ dependence gives an enhancement of the coefficients $X^{(u, d)}$ for $\tan \beta \approx 1$, but rapidly saturates for large values of $\tan \beta$. We will focus on the most conservative case $\tan \beta \gg 1$, but our results can be simply scaled by replacing $M_{H}$ with $M_{H}|\cos 2 \beta|$.

We first consider the bounds from the meson-antimeson mass differences, which depend only on the absolute value of the $X^{(u, d)}$ coefficients. The results are summarised in table $1 .{ }^{9}$ The entries in the table can be read in a twofold manner. On the one hand, we can derive absolute lower bounds on $M_{H}$ in the limit $s_{i j} \rightarrow 0\left(V_{R} \rightarrow 11\right)$. In such a limit, the $X^{(u, d)}$ coefficients are necessarily suppressed by at least one light Yukawa eigenvalue. As a result of the smallness of the Yukawa couplings of light quarks, the resulting constraints are not very stringent. The tightest bound is the one following from $\Delta M_{K}$, which implies $M_{H}>0.75 \mathrm{TeV}$.

On the other hand, comparing the numerical values (independent of $s_{i j}$ ) with the terms linear in $s_{i j}$ in the bounds of table 1, we deduce the size of the right-handed mixing angles for which the bound on $M_{H}$ becomes more stringent with respect to the one derived in the $V_{R}=1$ limit. In the kaon system this happens for $\left|s_{12}\right| \gtrsim 10^{-3}$, while in the $B_{d}$ system the right-handed mixing gives the leading effect already for $\left|s_{13}\right| \gtrsim 10^{-7}$.

For right-handed mixing angles equal to the corresponding left-handed CKM mixings - $\left|s_{12}\right|=\left|V_{u s}\right|,\left|s_{23}\right|=\left|V_{c b}\right|$, and $\left|s_{13}\right|=\left|V_{u b}\right|$ — we find comparable and quite strin-

\footnotetext{
${ }^{9}$ We use the updated list of bounds on the coefficients of $\Delta F=2$ operators from [70].
} 


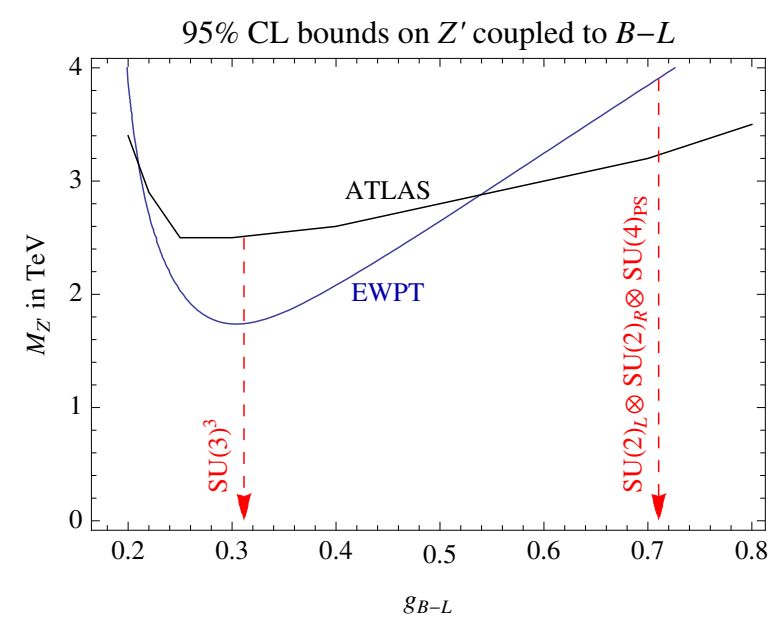

Figure 4. 95\% CL bounds on the $Z^{\prime}$ mass from electroweak precision tests (EWPT, blue curve) and from LHC-Run1 data (ATLAS, black curve) in extensions of the SM where $\mathrm{U}(1)_{B-L} \otimes \mathrm{SU}(2)_{R} \rightarrow$ $\mathrm{U}(1)_{Y}$. The red dashed vertical arrows indicate the predictions for $g_{B-L}$ coming from the nonabelian Pati-Salam and trinification models.

gent bounds on $M_{H}$ from $\Delta M_{K}\left(M_{H}>14 \mathrm{TeV}\right), \Delta M_{B_{s}}\left(M_{H}>19 \mathrm{TeV}\right)$, and $\Delta M_{B_{d}}$ $\left(M_{H}>14 \mathrm{TeV}\right)$.

However, as previously discussed, small values of the right-handed mixing angles are almost radiatively stable, and for the smallest natural mixing angles of $V_{R}$ estimated in eq. (5.18) the bound becomes

$$
M_{H} \gtrsim 3 \mathrm{TeV} .
$$

Considering now the CP-violating effects, if the $\left|s_{i j}\right|$ are close to the values that saturate the $\Delta M$ bounds, the flavour-dependent phases in $P_{u, d}$ and the phase $\phi$ in (5.19) are significantly constrained. These additional constraints are not particularly tight in the $B_{d, s}$ systems, where the bounds on $\mathrm{CP}$-conserving and CP-violating $\Delta F=2$ amplitudes are comparable in size, but are quite relevant in the kaon system. The experimental constraint on $\epsilon_{K}$ implies $\left|\phi_{1,2}^{q}\right|<4 \times 10^{-3}$, if $\left|s_{12}\right|$ is close to saturate the $\Delta M_{K}$ bound (barring cancellations among different contributions).

We finally mention that constraints from $\Delta F=1$ processes of the type $q_{i} \rightarrow q_{j} \ell^{+} \ell^{-}$ or $q_{i} \rightarrow q_{j} \gamma$ are not very stringent: in the first case $\left(q_{i} \rightarrow q_{j} \ell^{+} \ell^{-}\right)$the corresponding scalar operators are suppressed by light lepton masses, while in the second case $\left(q_{i} \rightarrow q_{j} \gamma\right)$ the transition is generated only beyond the tree level.

\subsection{New heavy $\mathrm{SU}(2)_{R}$ vectors}

In both $\mathrm{SU}(2)_{L} \otimes \mathrm{SU}(2)_{R} \otimes \mathrm{SU}(4)_{\mathrm{PS}}\left(\right.$ Pati-Salam) and $\mathrm{SU}(3)_{L} \otimes \mathrm{SU}(3)_{R} \otimes \mathrm{SU}(3)_{c}$ (trinification) the electroweak gauge group comes from $\mathrm{SU}(2)_{L} \otimes \mathrm{SU}(2)_{R} \otimes \mathrm{U}(1)_{B-L}$, with gauge couplings $g_{L}, g_{R}$ and $g_{B-L}$, respectively. The SM electroweak gauge couplings are denotes as $g_{2}=g_{L}$ and $g_{Y}=\sqrt{3 / 5} g_{1}$. Both models predicts extra heavy $W_{R}^{ \pm}$and $Z^{\prime}$ vectors. In both non-abelian models, hypercharge is obtained as a combination of the $B-L$ and of the $T_{3 R}$ vectors, and the hypercharge gauge coupling is reproduced as $1 / g_{Y}^{2}=1 / g_{R}^{2}+1 / 4 g_{B-L}^{2}$, 
i.e.

$$
g_{Y}=2 g_{B-L} \cos \theta_{B-L}=g_{R} \sin \theta_{B-L}
$$

Each model implies a specific value of its gauge couplings and in particular of $g_{B-L}$ :

$$
g_{B-L}=\sqrt{\frac{3}{8}} g_{3}, \quad g_{R}=\frac{g_{Y}}{\sqrt{1-2 g_{Y}^{2} / 3 g_{3}^{2}}} \approx 1.03 g_{Y} \quad \text { (Pati-Salam) }
$$

and

$$
g_{B-L}=\frac{g_{Y} g_{2}}{\sqrt{g_{Y}^{2}+g_{2}^{2}}}, \quad g_{R}=\frac{2 g_{Y} g_{2}}{\sqrt{3 g_{2}^{2}-g_{Y}^{2}}} \approx 1.22 g_{Y} \quad \text { (Trinification). }
$$

Bounds on heavy $\boldsymbol{Z}^{\prime}$ vectors. The angle $\tan \theta_{B-L}=2 g_{B-L} / g_{R}$ describes the mixing between the $B-L$ and $T_{3 R}$ vectors, in analogy to the weak mixing angle. The heavy $Z^{\prime}$ couples to SM fermions as

$$
\frac{g_{Y}\left[(B-L)-2 \cos ^{2} \theta_{B-L} Y\right]}{2 \sin \theta_{B-L} \cos \theta_{B-L}}
$$

LHC experiments directly searched for $Z^{\prime}$ vectors as di-lepton and di-jet resonances [71-74]. ATLAS reported the experimental bounds in terms of the relevant category of 'minimal' $Z^{\prime}$ vectors, which are combination of $B-L$ and $Y[75,76]$, such that we can extract from data the $95 \%$ C.L. bounds: $M_{Z^{\prime}}>3.2 \mathrm{TeV}$ for the Pati-Salam $Z^{\prime}\left(\tilde{g}_{Y} \approx-0.124\right.$ and $\tilde{g}_{B-L} \approx 0.99$ in the notations of $\left.[75,76]\right)$ and $M_{Z^{\prime}}>2.6 \mathrm{TeV}$ for trinification $\left(\tilde{g}_{Y} \approx-0.33\right.$ and $\tilde{g}_{B-L} \approx 0.51$ ).

Furthermore, $Z^{\prime}$ vectors are significantly constrained from precision electroweak data. Using the results of $[77,78]$, we performed a global fit of precision data finding the bound on the $Z^{\prime}$ mass as function of $g_{B-L}$ plotted in figure 4 . The figure also shows the special values of $g_{B-L}$ predicted by the two non-abelian models. The $95 \%$ C.L. bound is $M_{Z^{\prime}} \gtrsim 4 \mathrm{TeV}$ in Pati-Salam models and $M_{Z^{\prime}} \gtrsim 1.8 \mathrm{TeV}$ in trinification models.

Bounds on heavy $\boldsymbol{W}_{\boldsymbol{R}}$ vectors. The interactions of the $W_{R}^{ \pm}$bosons are described by

$$
\mathscr{L}_{W_{R}}=\frac{g_{R}}{\sqrt{2}}\left[\left(V_{R}\right)_{i j} \bar{u}_{R}^{i} \gamma_{\mu} d_{R}^{j}+\bar{\nu}_{R}^{i} \gamma_{\mu} \ell_{R}^{i}\right] W_{R}^{\mu}+\text { h.c. }
$$

where $V_{R}$ is the unitary matrix introduced in section 5.3.

LHC experiments searched for heavy $W_{R}^{ \pm}$gauge bosons. For the predicted values of $g_{R}$, see eqs. (5.24) and (5.25), CMS data [79] imply the bound ${ }^{10}$

$$
M_{W_{R}} \gtrsim 2.2 \mathrm{TeV}
$$

Furthermore, we can identify two sets of indirect bounds on $M_{W_{R}}$ : those from treelevel charged-current interactions, and those from one-loop FCNC processes. The latter are largely dominant if $V_{R}$ has a generic flavour structure, but evaporate when $V_{R}$ is

\footnotetext{
${ }^{10} \mathrm{~A} 3 \sigma$ excess is present in the electron channel at the value of the mass corresponding to the bound.
} 


\begin{tabular}{|cccccc|}
\hline Fields & spin & $\mathrm{U}(1)_{B-L}$ & $\mathrm{SU}(2)_{L}$ & $\mathrm{SU}(2)_{R}$ & $\mathrm{SU}(3)_{\mathrm{c}}$ \\
\hline$\left(\nu_{L}, e_{L}\right)$ & $1 / 2$ & -1 & $\overline{2}$ & 1 & 1 \\
$\left(\nu_{R}, e_{R}\right)$ & $1 / 2$ & +1 & 1 & 2 & 1 \\
$\left(u_{L}, d_{L}\right)$ & $1 / 2$ & $+\frac{1}{3}$ & $\overline{2}$ & 1 & 3 \\
$\left(u_{R}, d_{R}\right)$ & $1 / 2$ & $-\frac{1}{3}$ & 1 & 2 & $\overline{3}$ \\
$\phi$ & 0 & 0 & 2 & $\overline{2}$ & 1 \\
\hline
\end{tabular}

Table 2. Field content of extensions of the $\mathrm{SM}$ where $\mathrm{U}(1)_{Y}$ is embedded in $\mathrm{SU}(2)_{R} \times \mathrm{U}(1)_{B-L}$.

sufficiently close to a permutation matrix. If we assume that $V_{R}$ is such that the bounds in table 1 are satisfied for $M_{H} \leq 3 \mathrm{TeV}$, then the FCNC bounds on $M_{W_{R}}$ are automatically satisfied for $M_{W_{R}} \gtrsim 300 \mathrm{GeV}$. This happens because $W_{R}$-mediated FCNCs appear only at the one-loop level, and therefore the induced operators have an effective suppression scale $M_{\mathrm{eff}} \sim 4 \pi \times M_{W_{R}}$.

More specifically, integrating out $W_{R}$ at tree level leads to the following charged-current effective Lagrangian,

$$
\mathscr{L}_{\mathrm{eff}}^{\mathrm{CC}}=\frac{g_{R}^{2}}{2 M_{W_{R}}^{2}}\left[\left(\bar{\nu}_{R} \gamma_{\mu} \mu_{R}\right)\left(\bar{e}_{R} \gamma^{\mu} \nu_{R}\right)+\left(V_{R}\right)_{u d}\left(\bar{u}_{R} \gamma_{\mu} d_{R}\right)\left(\bar{e}_{R} \gamma^{\mu} \nu_{R}\right)+\cdots\right]+\text { h.c. }
$$

where we wrote explicitly the two most dangerous operators. The first term affects the determination of $G_{\mathrm{F}}$ from $\mu$ decays, while the second operator affects nuclear $\beta$ decays and LHC physics (see [80]). The right-handed nature of these effective operators implies that they do not interfere with the left-handed SM contributions. As a result, the correction to both $G_{\mathrm{F}}^{(\mu)}$ and nuclear $\beta$ decays is suppressed by $1 / M_{W_{R}}^{4}$,

$$
G_{\mathrm{F}}^{(\mu)} \rightarrow G_{\mathrm{F}}^{(\mu)}\left[1+\frac{g_{R}^{4} M_{W}^{4}}{g_{L}^{4} M_{W_{R}}^{4}}\right]^{1 / 2} \approx G_{\mathrm{F}}^{(\mu)}\left[1+1.9 \times 10^{-6}\left(\frac{1 \mathrm{TeV}}{M_{W_{R}}}\right)^{4}\right],
$$

and does not leads to stringent bounds on $M_{W_{R}}$. Moreover, if $\left(V_{R}\right)_{u d}=1$ the correction is almost universal in $G_{\mathrm{F}}^{(\mu)}$ and nuclear $\beta$ decays, thereby not affecting the most stringent low-energy test of charged-current weak interactions, namely the comparison between these two effective couplings.

\section{Towards a realistic weak-scale TAF theory}

In this section we will attempt to construct realistic models of a TAF theory at the weak scale, based on Pati-Salam or trinification gauge groups. We describe all fermions as 2component Weyl spinors and we use a short-hand notation in which the left-handed quark is denoted by $q_{L}$ and the conjugate right-handed quark by $q_{R}$ (with the symbol $c$ omitted). Moreover, for future convenience, we use a non-conventional assignment in which lefthanded quarks transforms as anti-doublets of $\mathrm{SU}(2)_{L}$. A summary of the quantum number assignments of SM particles is given in table 2 . 


\begin{tabular}{|c|c|c|c|c|c|c|}
\hline & Fields & spin & generations & $\mathrm{SU}(2)_{L}$ & $\mathrm{SU}(2)_{R}$ & $\mathrm{SU}(4)_{\mathrm{PS}}$ \\
\hline \multirow{4}{*}{ 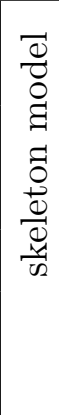 } & $\psi_{L}=\left(\begin{array}{cc}\nu_{L} & e_{L} \\
u_{L} & d_{L}\end{array}\right)$ & $1 / 2$ & 3 & $\overline{2}$ & 1 & 4 \\
\hline & $\psi_{R}=\left(\begin{array}{ll}\nu_{R} & u_{R} \\
e_{R} & d_{R}\end{array}\right)$ & $1 / 2$ & 3 & 1 & 2 & $\overline{4}$ \\
\hline & $\phi_{R}$ & 0 & 1 & 1 & 2 & $\overline{4}$ \\
\hline & $\phi=\left(\begin{array}{cc}H_{U}^{0} & H_{D}^{+} \\
H_{U}^{-} & H_{D}^{0}\end{array}\right)$ & 0 & 1 & 2 & $\overline{2}$ & 1 \\
\hline \multirow{4}{*}{ 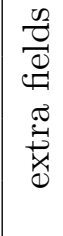 } & $\psi$ & $1 / 2$ & $N_{\psi} \leq 3$ & 2 & $\overline{2}$ & 1 \\
\hline & $Q_{L}$ & $1 / 2$ & 2 & 1 & 1 & 10 \\
\hline & $Q_{R}$ & $1 / 2$ & 2 & 1 & 1 & $\overline{10}$ \\
\hline & $\Sigma$ & 0 & 1 & 1 & 1 & 15 \\
\hline
\end{tabular}

Table 3. Field content of the skeleton Pati-Salam model (upper box) and the extra fields needed for a possible realistic TAF model (lower box).

\subsection{Pati-Salam SU(2) $)_{L} \otimes \mathrm{SU}(2)_{R} \otimes \mathrm{SU}(4)_{\mathrm{PS}}$}

Skeleton model. The fermion and scalar field content of a 'skeleton' Pati-Salam model, summarised in the upper box of table 3 , is described by

- The SM fermions are contained in the Weyl fermions $\psi_{L} \oplus \psi_{R}$.

- The scalar $\phi_{R}$, in the same representation as $\psi_{R}$, can get a vev $v_{R}$ in its canonicallynormalised $\operatorname{Re} \tilde{\nu}_{R}$ component (we denote the entries as $\phi_{R}$ as those of $\psi_{R}$, adding a tilde symbol, like in supersymmetric models) breaking $G_{224} \rightarrow G_{\mathrm{SM}}$. Then, the scalars $\tilde{e}_{R}, \operatorname{Im} \tilde{\nu}_{R}$ and $\tilde{u}_{R}$ are respectively eaten by the vectors $W_{R \mu}^{ \pm}, Z_{\mu}^{\prime}$ and by the 6 leptoquarks $W_{\mu}^{\prime}$ (coming from $\left.\mathrm{SU}(4)_{\mathrm{PS}} / \mathrm{SU}(3)_{c}\right)$, which acquire mass

$$
M_{W_{R}}^{2}=\frac{g_{R}^{2}}{2} v_{R}^{2}, \quad M_{Z^{\prime}}^{2}=\frac{2 g_{R}^{2}+3 g_{4}^{2}}{4} v_{R}^{2}, \quad M_{W^{\prime}}^{2}=\frac{g_{4}^{2}}{2} v_{R}^{2}
$$

- The SM Higgs is contained in the scalar $\phi$, in the $\left(2_{L}, \overline{2}_{R}\right)$ representation, which is real given that $2_{L}$ and $\overline{2}_{R}$ are pseudo-real, with $\phi^{c} \equiv \epsilon^{T} \phi^{*} \epsilon$ also transforming as $\left(2_{L}, \overline{2}_{R}\right)$. One could impose the reality condition $\phi= \pm \phi^{c}$, and the representation $\phi$ would contain only one Higgs doublet with a single Higgs mass term. However, the left-right symmetry would constrain the Yukawa interactions to give identical masses for up and down quarks. It is phenomenologically more interesting to treat $\phi$ as a complex scalar field $\phi$, containing two Higgs doublets. In this case, there are two Higgs mass terms, $\frac{m_{1}^{2}}{2} \operatorname{Tr}\left(\phi^{\dagger} \phi\right)+\operatorname{Re} \frac{m_{2}^{2}}{4} \operatorname{Tr}\left(\phi^{\dagger} \phi^{c}\right)$ with mass eigenvalues $m_{1}^{2} \pm m_{2}^{2}$. 
After symmetry breaking, the Pati-Salam gauge couplings $g_{L}, g_{R}, g_{4}$ are related to the SM gauge couplings $g_{3}, g_{2}$, and $g_{Y}=\sqrt{3 / 5} g_{1}$ by

$$
g_{L}=g_{2}, \quad g_{R}=\frac{g_{Y}}{\sqrt{1-2 g_{Y}^{2} / 3 g_{3}^{2}}}, \quad g_{4}=g_{3} .
$$

The Yukawa couplings of the Pati-Salam skeleton model are

$$
-\mathscr{L}_{Y}=Y \psi_{R} \psi_{L} \phi+Y_{c} \psi_{R} \psi_{L} \phi^{c}+\text { h.c. }
$$

As discussed in section 5.3 the two-Higgs structure allows for independent structures of the up-quark and down-quark Yukawa matrices $Y_{U}$ and $Y_{D}$. However, in view of quark-lepton unification, the skeleton model predicts $Y_{E}=Y_{D}$, as in $\mathrm{SU}(5)$, and $Y_{N}=Y_{U}$, as in $\mathrm{SO}(10)$, where $Y_{N}$ is the Yukawa matrix of neutrinos.

Minimal extensions. There are at least two ways of avoiding the wrong quark-lepton mass predictions with the addition of new fields.

- Foot [81] proposed adding the vector-like fermions $\psi$ (in the same representation as the scalar $\phi$, see table 3, containing a SM lepton doublet and an anti-lepton doublet) and the scalar $\phi_{L}$ (in the same representation as the fermion $\psi_{L}$ ). In this way the Yukawa interactions are extended to

$$
-\mathscr{L}_{Y}=Y \psi_{R} \psi_{L} \phi+Y_{c} \psi_{R} \psi_{L} \phi^{c}+Y_{N} \psi_{L} \psi \phi_{R}+Y_{L} \psi \psi_{R} \phi_{L}+\text { h.c. }
$$

The third term provides a mass term $Y_{N}\left\langle\phi_{R}\right\rangle$ pairing the lepton doublet in $\psi_{L}$ with the anti-lepton doublet in $\psi$, while the lepton doublet in $\psi$ remains massless before EW breaking. In this way, the SM quark doublet is embedded in $\psi_{L}$, while the SM lepton doublet is embedded in $\psi$, and lepton-quark unification is evaded in the left-handed sector. This breaking of lepton-quark unification also relaxes the bounds (discussed in section 6.2) on the vector leptoquark mass $M_{W^{\prime}}$, which can safely be about a few $\mathrm{TeV}$ [81]. After EW breaking, the SM leptons acquire a mass $Y_{L}\left\langle\phi_{L}\right\rangle$ through the last term of the Yukawa interactions in eq. (6.4). Note that this mechanism requires the absence of a mass term $\psi \psi$, which is allowed by the gauge symmetry.

- Volkas [82] proposed adding the fermions $Q_{L} \oplus Q_{R}$ in the $10 \oplus \overline{10}$ of $\mathrm{SU}(4)_{\mathrm{PS}}$ (see table 3). These fields contain a vector-like copy of a right-handed lepton $e_{R}$, of a right-handed quark $d_{R}$, and of an exotic quark in a colour sextuplet. The Yukawa couplings and fermion mass terms are ${ }^{11}$

$$
Y \psi_{L} \psi_{R} \phi+Y_{c} \psi_{L} \psi_{R} \phi^{c}+Y_{Q} \psi_{R} Q_{L} \phi_{R}+M Q_{L} Q_{R}+\text { h.c. }
$$

The mass term $Y_{Q}\left\langle\phi_{R}\right\rangle$ induces a mass mixing between light and heavy states in the $e_{R}$ and $d_{R}$ sectors, which differ by a group-theoretical factor $\sqrt{2}$. As a consequence, the unwanted relation $Y_{D}=Y_{E}$ is avoided. Even with a single $Q_{L} \oplus Q_{R}$ pair, the quark and charged-lepton masses can be made to agree with data. Similarly, by adding a fermion singlet [82], one can avoid the unwanted prediction in the neutrino sector, $Y_{N}=Y_{U}$.

\footnotetext{
${ }^{11}$ Volkas presented a slightly different model with a real $\phi$.
} 
In both versions of the Pati-Salam model, gauge and Yukawa interactions are invariant under an accidental $\mathrm{U}(1)_{B^{\prime}}$ global symmetry, defined by the following charge assignments: $B^{\prime}\left(\psi_{L}\right)=B^{\prime}\left(\phi_{L}\right)=1, B^{\prime}\left(\psi_{R}\right)=B^{\prime}\left(\phi_{R}\right)=-1, B^{\prime}(\psi)=B^{\prime}(\phi)=0$, $B^{\prime}\left(Q_{L}\right)=-B^{\prime}\left(Q_{R}\right)=2$. The vevs of $\phi_{R}$ and $\phi_{L}$ break spontaneously both $\mathrm{SU}(4)_{\mathrm{PS}}$ and $\mathrm{U}(1)_{B^{\prime}}$, leaving unbroken a new $\mathrm{U}(1)$ global symmetry given by $(3 / 4)\left[B^{\prime}+(B-L)\right]$ which, for SM particles, corresponds to baryon number. This symmetry protects proton stability, and prevents the appearance of a massless state related to the spontaneous breaking of $\mathrm{U}(1)_{B^{\prime}}$.

We analysed the two minimal realistic Pati-Salam models finding that, while the gauge couplings and the Yukawa couplings have appropriate TAF solutions, this is never the case for the quartic couplings in the scalar potential. Indeed the most general quartic scalar potential is

$$
V\left(\phi, \phi_{R}\right)=V_{\phi_{R}}+V_{\phi}+V_{\phi \phi_{R}}
$$

or, adding the scalar $\phi_{L}$ proposed by Foot:

$$
V\left(\phi, \phi_{R}, \phi_{L}\right)=V_{\phi_{R}}+V_{\phi_{L}}+V_{\phi}+V_{\phi \phi_{L}}+V_{\phi \phi_{R}}+V_{\phi_{L} \phi_{R}}+V_{\phi_{L} \phi_{R}}^{B}
$$

which contains 18 real couplings and 6 complex couplings. The various potential terms and the corresponding quartic couplings are defined in eq. (A.14). The RGE for the quartics form a large system, listed in appendix A. ${ }^{12}$ We do not find any TAF solution unless the $\beta$-function coefficient of $g_{4}$ is artificially reduced down to unacceptably small values.

\subsection{A TAF Pati-Salam model}

By considering non-minimal Pati-Salam models we found TAF models with 3 generations, which seems to be the maximum allowed in the present context. ${ }^{13}$ Table 3 describes the matter content of the model, which employs

- the matter content of the skeleton Pati-Salam model (upper box),

- $N_{\psi} \leq 3$ generations of the $\psi$ fermions proposed by Foot [81],

- $N_{Q}=2$ generations of the $Q_{L} \oplus Q_{R}$ fermion proposed by Volkas [82],

- a scalar $\Sigma$ in the adjoint of $\mathrm{SU}(4)_{\mathrm{PS}}$.

\footnotetext{
${ }^{12}$ It is interesting to observe that in this model where the scalar $\phi_{L}$ is present, the coupling $\lambda_{B}$ in $V_{\phi_{L} \phi_{R}}^{B}$ is the only coupling that violates baryon number. Therefore, it is only multiplicatively renormalised and its $\beta$-function must be proportional to the coupling itself $\left(d \lambda_{B} / d \ln \mu \propto \lambda_{B}\right.$, as shown in appendix A). We found that the TAF conditions generally imply that $\lambda_{B}$ must vanish, either asymptotically or identically, depending on the TAF solutions for the other couplings entering the $\beta$-function of $\lambda_{B}$. Similarly, TAF often requires that $\mathrm{CP}$-violating quartics must vanish.

${ }^{13}$ A Pati-Salam TAF model with 2 generations was build by Kalashnikov in 1977 [56]. However, the model is incompatible with flavour data and cannot be extended to 3 generations because gauge couplings would no longer be asymptotically free. We confirm most of his results, although the author of [56] missed the existence of the quartics in our eq. (A.21), which could have changed the result. Its inclusion modifies the TAF conditions, and the model admits TAF solutions, including one with asymptotically positive potential.
} 
Upper bounds $N_{\psi} \leq 3$ and $N_{Q} \leq 2$ arise from the request that gauge couplings are asymptotically free:

$$
b_{L}=3-\frac{2}{3} N_{\psi}, \quad b_{R}=\frac{7}{3}-\frac{2}{3} N_{\psi}, \quad b_{4}=\frac{29}{3}-4 N_{Q} .
$$

The extra fields allow for a realistic fermion spectrum as described in section 6.1 and, at the same time, modify the RG running of the scalar quartics in such a way that TAF solutions are found for $N_{Q}=2$.

The most general Yukawa interactions are ${ }^{14}$

$$
-\mathscr{L}_{\mathrm{Y}}=Y \phi \psi_{L} \psi_{R}+Y_{c} \phi^{c} \psi_{L} \psi_{R}+Y_{N} \psi_{L} \psi \phi_{R}+Y_{Q} \psi_{R} Q_{L} \phi_{R}+Y_{\Sigma} Q_{L} Q_{R} \Sigma+\text { h.c. }
$$

The Yukawa coupling $Y_{Q}$ gives mass to $Q_{L, R}$, once $\Sigma$ acquires a vev. The most general scalar potential is

$$
V\left(\phi, \phi_{R}, \Sigma\right)=V_{\phi_{R}}+V_{\phi}+V_{\phi \phi_{R}}+V_{\Sigma}+V_{\phi \Sigma}+V_{\phi_{R} \Sigma}
$$

where the various terms are defined in eq. (A.14). The RGE are listed in appendix A. The simplest TAF model corresponds to $N_{\psi}=0$ (no $\psi$ field and so $Y_{N}$ is absent). TAF solutions for the quartics are found only if the gauge and Yukawa couplings are on the following fixed-flow

\begin{tabular}{|c|ccccccc|}
\hline & $\tilde{g}_{L \infty}^{2}$ & $\tilde{g}_{R \infty}^{2}$ & $\tilde{g}_{4 \infty}^{2}$ & $\tilde{Y}_{\infty}^{2}$ & $\tilde{Y}_{c \infty}^{2}$ & $\tilde{Y}_{Q \infty}^{2}$ & $\tilde{Y}_{\Sigma \infty}^{2}$ \\
\hline Fixed-flow & $3 / 5$ & $1 / 3$ & $3 / 7$ & 0.432 & 0 & 0.909 & 3.454 \\
$M$-Eigenvalues & - & - & - & + & + & + & + \\
\hline
\end{tabular}

There are 4 positive eigenvalues of the $M$ matrix, hence 4 Yukawa couplings are univocally predicted at low energy. The IR prediction $Y_{c}=0$ is incompatible with a realistic quarkmass spectrum, but can be evaded in the more complicated TAF model with $N_{\psi}>0$ and extra Yukawa couplings described in appendix A.

\begin{tabular}{|c|c|c|c|c|c|c|c|c|c|c|c|c|c|c|c|}
\hline$N_{+}$ & $\lambda_{\Sigma \phi_{R}}{ }$ & $\lambda_{\Sigma \phi 1}$ & $\lambda_{\Sigma \phi_{R}{ }^{2}}$ & $\lambda_{\Sigma \phi 2}$ & $\lambda_{R 1}$ & $\lambda_{R 2}$ & $\lambda_{\Sigma 1}$ & $\lambda_{\Sigma 2}$ & $\lambda_{1}$ & $\lambda_{2}$ & $\lambda_{3}$ & $\lambda_{4}$ & $\lambda_{\phi_{R} \phi 1}$ & $\lambda_{\phi_{R} \phi 2}$ & $\lambda_{\phi}{ }_{R} \phi 3$ \\
\hline 10 & -0.023 & -0.127 & -1.067 & 0 & -0.017 & 0.082 & 0.374 & -1.031 & 0 & -0.015 & -0.037 & -0.057 & 0.172 & 0 & -0.322 \\
\hline 8 & 0.210 & 0 & -1.174 & -0.024 & 0.036 & 0.042 & 0.661 & -1.621 & -0.033 & 0.018 & -0.034 & -0.013 & 0.210 & 0 & -0.344 \\
\hline 10 & -0.040 & -0.018 & -1.021 & 0 & 0 & 0.056 & 0.313 & -0.973 & -0.028 & -0.031 & 0 & -0.095 & 0.195 & 0 & -0.381 \\
\hline 8 & -0.036 & -0.053 & -1.036 & 0 & 0 & 0.068 & 0.341 & -1.009 & -0.051 & 0.019 & -0.031 & 0 & 0.197 & 0 & -0.357 \\
\hline 6 & 0 & -0.184 & -1.110 & 0 & -0.028 & 0.095 & 0.404 & -1.033 & 0.022 & -0.022 & 0 & -0.101 & 0.152 & 0 & -0.277 \\
\hline 8 & 0.212 & -0.129 & -1.197 & 0 & 0.033 & 0.047 & 0.660 & -1.585 & 0 & 0.027 & 0 & 0 & 0.192 & 0 & -0.327 \\
\hline 7 & 0.206 & -0.181 & -1.233 & 0.036 & 0.021 & 0.059 & 0.656 & -1.513 & 0 & 0 & -0.024 & -0.054 & 0.194 & 0 & -0.286 \\
\hline 9 & 0.215 & -0.077 & -1.179 & 0 & 0.038 & 0.040 & 0.662 & -1.616 & 0 & -0.029 & 0 & -0.106 & 0.197 & 0 & -0.347 \\
\hline 8 & -0.027 & -0.095 & -1.059 & 0 & -0.021 & 0.083 & 0.374 & -1.045 & -0.085 & 0 & 0 & -0.032 & 0.211 & 0 & -0.324 \\
\hline 9 & 0.185 & 0.054 & -1.165 & -0.089 & 0.037 & 0.039 & 0.621 & -1.516 & -0.052 & 0.013 & -0.017 & -0.023 & 0.219 & 0 & -0.354 \\
\hline 9 & 0.139 & 0.164 & -1.151 & 0 & 0.016 & 0.062 & 0.599 & -1.475 & 0.024 & 0 & 0 & 0 & 0.218 & 0 & -0.312 \\
\hline 10 & 0.185 & 0.054 & -1.165 & 0.089 & 0.037 & 0.039 & 0.621 & -1.516 & -0.052 & 0.013 & 0.017 & -0.023 & 0.219 & 0 & -0.354 \\
\hline 9 & 0.194 & 0.046 & -1.174 & 0.030 & 0.029 & 0.051 & 0.653 & -1.599 & 0.010 & -0.016 & 0.037 & -0.059 & 0.206 & 0 & -0.326 \\
\hline 9 & 0.167 & 0.149 & -1.156 & 0.060 & 0.032 & 0.044 & 0.607 & -1.488 & -0.015 & -0.019 & 0.026 & -0.062 & 0.222 & 0 & -0.348 \\
\hline 9 & 0.167 & 0.149 & -1.156 & -0.060 & 0.032 & 0.044 & 0.607 & -1.488 & -0.015 & -0.019 & -0.026 & -0.062 & 0.222 & 0 & -0.348 \\
\hline
\end{tabular}

The 19 quartics admit 25 different TAF solutions. If we set to zero the $4 \mathrm{CP}$-violating couplings, we find 15 TAF solutions for the $15 \mathrm{CP}$-conserving quartics, given by

\footnotetext{
${ }^{14}$ With the addition of three generations of massless fermion singlets $\psi_{1} \sim(1,1,1)$, the Yukawa couplings $y_{1} \psi_{1} \psi_{R} \phi_{R}^{\dagger}$ satisfy the TAF condition and allow us to obtain realistic neutrino masses avoiding the $Y_{N}=Y_{U}$ relation. An extra $\psi_{\Sigma} \sim(1,1,15)$ could play a similar role or could be identified with the Dark Matter, if its Yukawa couplings vanish.
} 
The first column shows the number of positive eigenvalues of the $M$ matrix, i.e. the number of quartics predicted at low energy. All these solutions give gauge, Yukawa, and quartic couplings that can be extrapolated up to infinite energy without hitting any Landau pole. However, all these solutions correspond to theories with metastable vacua, because the scalar potential has always some negative directions in the asymptotic region of large fields.

Flavour bounds from vector leptoquarks. The experimental bounds on heavy Higgs bosons and on $W_{R}$ and $Z^{\prime}$ gauge bosons have been discussed in sections 5.3 and 5.4. The Pati-Salam gauge group also contains massive vector leptoquarks $W_{\mu}^{\prime}$, of charge $\pm 2 / 3$, corresponding to the broken generators in $\mathrm{SU}(4)_{\mathrm{PS}} / \mathrm{SU}(3)_{c}$. The gauge bosons $W_{\mu}^{\prime}$ are coupled to a right-handed current involving $d_{R}$ and $e_{R}$ (since these two fields are unified in $\psi_{R}$, see table 3 ), and to a left-handed current involving $q_{L}$ and $\ell_{L}$ doublets (only in versions of the model in which $q_{L}$ and $\ell_{L}$ are unified in the field $\psi_{L}$ ).

When both left-handed and right-handed interactions are present, data on $\pi \rightarrow e \nu$ give the strong constraint [83]

$$
M_{W^{\prime}}>250 \mathrm{TeV} \quad \text { (Pati-Salam with } q_{L}-\ell_{L} \text { and } d_{R^{-}} e_{R} \text { unification). }
$$

This bound can be avoided in the models discussed in section 6.1 where the SM left-handed leptons $\ell_{L}$ are contained in the field $\psi$, while the quark doublet $q_{L}$ is in $\psi_{L}$. In this case, the gauge bosons $W_{\mu}^{\prime}$ are coupled only to right-handed currents of SM fermions. As discussed in appendix B, the bounds from right-handed interactions can be significantly relaxed with an appropriate flavour structure and, under the most favourable assumptions, they give

$$
M_{W^{\prime}}>8.8 \mathrm{TeV} \quad \text { (Pati-Salam with } d_{R^{-}} e_{R} \text { unification). }
$$

Taking into account the relation $M_{W_{R}} \approx g_{Y} M_{W^{\prime}} / g_{3}$, the bound in eq. (6.13) implies $M_{W_{R}}>2.7 \mathrm{TeV}$, which is comparable to those from direct $W_{R}$ searches (see section 5.4).

However, the Pati-Salam TAF models that we discovered do not contain the scalar $\phi_{L}$ and thus lead to $q_{L}-\ell_{L}$ unification. This implies that these models suffer from the strong bound of eq. (6.12) and, consequently, from an unnaturally high degree of fine-tuning. We have not found Pati-Salam TAF models containing the scalar $\phi_{L}$.

\subsection{Trinification $\mathrm{SU}(3)_{L} \otimes \mathrm{SU}(3)_{R} \otimes \mathrm{SU}(3)_{c}$}

Trinificaton is often considered as a unification model, after imposing a permutation symmetry among the three $\mathrm{SU}(3)$ factors that forces the gauge couplings $g_{L}, g_{R}, g_{c}$ to be equal. While the permutation symmetry is respected by the SM fermions (see table 4), it requires the addition of extra Higgs bosons with interactions that break baryon number. We do not impose any permutation symmetry in order to insure that trinification interactions at the weak scale conserve baryon number. The relation between the trinification gauge coupling constants $\left(g_{L}, g_{R}, g_{c}\right)$ and those of the $\operatorname{SM}\left(g_{3}, g_{2}, g_{Y}=\sqrt{3 / 5} g_{1}\right)$ is

$$
g_{L}=g_{2}, \quad g_{R}=\frac{2 g_{2} g_{Y}}{\sqrt{3 g_{2}^{2}-g_{Y}^{2}}}, \quad g_{c}=g_{3} .
$$




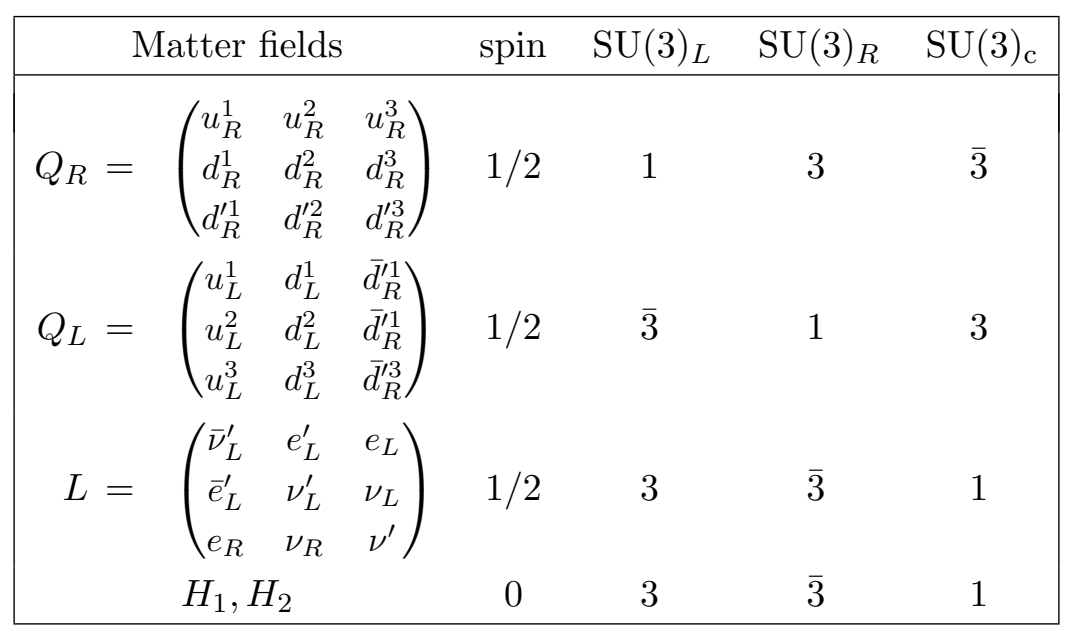

Table 4. Field content of the minimal trinification model. Primed fermions correspond to new states beyond the SM.

We consider the minimal model with the matter content summarised in table 4 . Since quarks are not unified with leptons, trinification is safer than Pati-Salam from the point of view of flavour. Each generation of $Q_{R} \oplus Q_{L} \oplus L$ contains 27 chiral fermions that decompose under the SM gauge group as the usual 15 SM fermions, plus a vector-like lepton doublet, a vector-like right-handed down quark, and two singlets (denoted as primed fermions in table 4). These states correspond to the irreducible representation 27 of $\mathrm{E}(6)$.

A single Higgs $H_{1}$ in the $\left(3_{L}, \overline{3}_{R}\right)$ representation contains 3 Higgs doublets. The vev $\left\langle H_{1}\right\rangle_{33}=V_{1} / \sqrt{2}$ breaks $\mathrm{SU}(3)^{3}$ to $\mathrm{SU}(2)_{L} \otimes \mathrm{SU}(2)_{R} \otimes \mathrm{U}(1)_{B-L} \otimes \mathrm{SU}(3)_{c}$. Two Higgs doublets and one singlet are eaten by the 9 components of the vector bosons that acquire mass

$$
M_{H_{L}}^{2}=\frac{g_{L}^{2}}{2} V_{1}^{2}, \quad M_{H_{R}}^{2}=\frac{g_{R}^{2}}{2} V_{1}^{2}, \quad M_{Z^{\prime}}^{2}=\frac{2}{3}\left(g_{L}^{2}+g_{R}^{2}\right) V_{1}^{2} .
$$

The massive $Z^{\prime}$ corresponds to the combination of gauge bosons $g_{L} A_{L \mu}^{8}-g_{R} A_{R \mu}^{8}$. The bound on its mass is in the range 2-6 TeV, depending on the $Z^{\prime}$ charge of the SM Higgs. The gauge boson of $B-L$ corresponds to $g_{R} A_{L \mu}^{8}+g_{L} A_{R \mu}^{8}$ with $g_{B-L}=(\sqrt{3} / 2) g_{R} g_{L} / \sqrt{g_{R}^{2}+g_{L}^{2}}$.

A second scalar is needed to break the left-right symmetry. This is accomplished by a Higgs $H_{2}$ in the $\left(3_{L}, \overline{3}_{R}\right)$ representation with vev $\left\langle H_{2}\right\rangle_{32}=V_{2} / \sqrt{2}$. The 12 components of the massive vectors can be grouped into the complex doublet $H_{L}$, which transforms as $\left(2_{L}, 1 / 2_{Y}\right) \oplus\left(\overline{2}_{L},-1 / 2_{Y}\right)$ under the $\mathrm{SM} \mathrm{SU}(2)_{L} \times \mathrm{U}(1)_{Y}$, two electrically-charged and one neutral complex gauge bosons $H_{R}^{ \pm}, W_{R} \sim\left(1_{L}, 1_{Y}\right) \oplus\left(1_{L},-1_{Y}\right)$ and $H_{R}^{0} \sim\left(1_{L}, 0_{Y}\right) \oplus\left(1_{L}, 0_{Y}\right)$, and two kinds of $Z^{\prime}$ gauge bosons. Their masses are given by

$$
M_{H_{L}}^{2}=\frac{g_{L}^{2}}{2}\left(V_{1}^{2}+V_{2}^{2}\right) \quad M_{H_{R}^{ \pm}}^{2}=\frac{g_{R}^{2}}{2}\left(V_{1}^{2}+V_{2}^{2}\right), \quad M_{H_{R}^{0}}^{2}=\frac{g_{R}^{2}}{2} V_{1}^{2}, \quad M_{W_{R}}^{2}=\frac{g_{R}^{2}}{2} V_{2}^{2} .
$$

The two $Z^{\prime}$ have a mass matrix which, in the limit $V_{2} \ll V_{1}$, leads to a heavier state with mass as in eq. (6.15), and a lighter $B-L$ gauge boson with mass

$$
M_{B-L}^{2} \simeq \frac{\left(g_{R}^{2}-2 g_{L}^{2}\right)^{2}}{6\left(g_{L}^{2}+g_{R}^{2}\right)} V_{2}^{2}
$$


As shown in figure 4 , the $B-L$ gauge boson is subjected to the bound $M_{B-L} \gtrsim 2.6 \mathrm{TeV}$ from ATLAS. The bounds from flavour processes mediated by the new gauge bosons are much milder than in the Pati-Salam models because no dangerous leptoquark interactions are present.

The SM Yukawa couplings are obtained from the $\mathrm{SU}(3)^{3}$-invariant interactions

$$
-\mathscr{L}_{Y}=\sum_{i=1}^{2}\left(y_{Q i} Q_{L} Q_{R} H_{i}+\frac{y_{L i}}{2} L L H_{i}^{*}\right)+\text { h.c. }
$$

Similarly to the case discussed in section 5.3, bounds from flavour processes can be kept under control because there are only two independent Yukawa matrices and therefore small quark masses suppress the new flavour interactions. However, this raises the problem of generating sufficiently large masses for the new fermions. The new (primed) fermions get mass from eq. (6.18), once the vev $V_{1}$ is generated, while mixing mass terms between new fermions and SM quarks/leptons are induced by the smaller vev $V_{2}$. Since all these terms are proportional to SM Yukawa couplings, the new fermions turn out to be too light, unless $V_{1}$ is unnaturally large. Additional fields and interactions are needed to construct realistic models.

Let us turn to the issue of the TAF properties of trinification models. We start by considering the simple, albeit unrealistic, case of a Higgs sector made of a single $H=H_{1}$ in the $\left(3_{L}, \overline{3}_{R}\right)$ with the most general quartic potential

$$
V=\lambda_{a} \operatorname{Tr}\left(H^{\dagger} H\right)^{2}+\lambda_{b} \operatorname{Tr}\left(H^{\dagger} H H^{\dagger} H\right) .
$$

The one-loop RGE for gauge, Yukawa, and quartic couplings are given in eq. (C.5) and admit two different TAF solutions:

\begin{tabular}{|c|ccccccc|}
\hline & $\tilde{g}_{L \infty}^{2}$ & $\tilde{g}_{R \infty}^{2}$ & $\tilde{g}_{c \infty}^{2}$ & $\tilde{y}_{Q \infty}^{2}$ & $\tilde{y}_{L \infty}^{2}$ & $\tilde{\lambda}_{a \infty}$ & $\tilde{\lambda}_{b \infty}$ \\
\hline Fixed-flow & $2 / 9$ & $2 / 9$ & $1 / 5$ & 0 & $23 / 54$ & 0.1628 & -0.1732 \\
$M$-Eigenvalue & - & - & - & - & + & - & + \\
\hline Fixed-flow & $2 / 9$ & $2 / 9$ & $1 / 5$ & 0 & $23 / 54$ & -0.0026 & -0.0087 \\
$M$-Eigenvalue & - & - & - & - & + & - & - \\
\hline
\end{tabular}

The potential $V$ is definite positive for $\lambda_{a} \geq-\lambda_{b}$ (for $\lambda_{b}<0$ ) and $3 \lambda_{a} \geq-\lambda_{b}$ (for $\lambda_{b}>0$ ). Both fixed-flows in eq. (6.20) violate the stability condition for the potential. The RGE flow can produce spontaneous symmetry breaking at low energy à la Coleman-Weinberg from a potential with no dimensional parameters.

Next, we consider the model with two Higgs multiplets $H_{1}$ and $H_{2}$ in the $\left(3_{L}, \overline{3}_{R}\right)$. The scalar quartic potential is given in eq. (C.7) and contains 14 real couplings and 6 phases. The relevant one-loop RGE are reported in appendix C. The gauge couplings and the Yukawa admit TAF solutions, but we do not find a TAF solution for the quartics. ${ }^{15}$

\footnotetext{
${ }^{15} \mathrm{TAF}$ solutions appear if the $\mathrm{SU}(3)_{c} \beta$ function coefficient is reduced to $b_{3}=1 / 6$. However it is impossible to obtain such value by adding fermionic multiples.
} 
The next step is to complicate the Higgs sector, with the twofold aim of recovering TAF and of generating sufficiently large masses for the new fermions. One possibility is to consider one $H$ and one $\Sigma_{R}$, adjoint under $\mathrm{SU}(3)_{R}$. A vev in its 23 component breaks $\mathrm{SU}(3)^{3}$ to the SM group. We also add one vector-like fermion $Q_{R}^{\prime} \oplus \bar{Q}_{R}^{\prime}$ which, in the model with a single $H$ is needed to obtain different masses for top and bottom quarks via right-handed quark mixing induced by $Q_{R}^{(\prime)} \bar{Q}_{R}^{\prime} \Sigma$ Yukawa couplings. A TAF solution exists only for a single generation of $Q_{R}^{\prime} \oplus \bar{Q}_{R}^{\prime}$, which is not sufficient to produce a realistic fermionic mass spectrum. Once we add two or more generations of $Q_{R}^{\prime} \oplus \bar{Q}_{R}^{\prime}$, the gauge group is no longer asymptotically free. In conclusion, we were not able to identify a TAF trinification model with a realistic flavour structure.

\section{Conclusions}

The main point of this paper is to single out the implications of two severe difficulties of theories trying to solve the Higgs naturalness problem by having the weak scale as the only effective source of breaking of scale invariance at the classical level.

The first well-known problem is gravity. The hope that transplanckian dynamics can miraculously cure the Higgs sensitivity to $M_{\mathrm{Pl}}$ seems to us unrealistic. In the absence of special symmetries like supersymmetry, any short-distance modification of general relativity is expected to affect the quantum corrections to the Higgs mass. Delaying such modifications to energy scales as high as $M_{\mathrm{Pl}}$ makes the problem too acute to leave us with any reasonable hope that new dynamics can rescue the situation: the problem must be tackled at energies lower than $M_{\mathrm{Pl}}$. From this line of reasoning, we have derived our first conclusion: in the context of theories with no dynamical protection of the Higgs mass, naturalness requires a premature modification of gravity, at scales no larger than $\Lambda_{G} \sim 4 \pi\left(M_{h} M_{\mathrm{Pl}}\right)^{1 / 2} \sim 10^{11} \mathrm{GeV}$. In this paper we have called softened gravity a theory in which the communication between gravity and the SM sector remains weak at any scale larger than $\Lambda_{G}$.

The second problem, less explored in the literature, is that the quantum theory necessarily breaks scale invariance. In the SM, the problem manifests itself in the form of the Landau pole for the hypercharge gauge coupling. In softened versions of gravity, this dynamically-generated scale brings back the naturalness problem. To cure this problem one needs to extend the SM, below a few $\mathrm{TeV}$, into a TAF theory. We have shown that the construction of such extensions is possible, but only at a high price: many new particles around the $\mathrm{TeV}$ scale are needed, as well as elaborate model-building to avoid phenomenological constraints, especially related to the flavour sector.

So our second (surprising) conclusion is that theories intended to deal with naturalness without new dynamics in the TeV range actually need a large number of new particles around the TeV scale. This reopens the usual can of worms with the phenomenological difficulties in satisfying constraints from collider searches and flavour processes that plague traditional approaches to naturalness, like supersymmetry or composite Higgs. Indeed, we find that the degree of tuning in the models we constructed is typically worse than in traditional approaches.

Our two conclusions are based on common intuition derived from effective field theory and dimensional analysis. Only in special setups that defy such usual intuition, could our 
conclusions be proved wrong. The only example of such a setup we are aware of is the use of anthropic arguments in the multiverse. We cannot exclude the existence of other theoretical setups that evade our conclusions.

The positive side of our result is that the class of theories we have considered is not at all elusive, but can be tested at high-energy colliders. While the next probe comes from Run-2 LHC, the existing constraints on new-particle masses from rare processes suggest that a $100-\mathrm{TeV}$ future collider is better positioned to explore the full parameter space. The other interesting aspect is that the experimental signals from softened-gravity models are distinct from those coming from traditional schemes with dynamical explanations of naturalness, but also differ from anthropic solutions, which are likely to have no light scalar particles other than the Higgs.

In a more technical vein, another result of our paper is the development of a systematic procedure to derive the asymptotic behaviour of coupling constants in a perturbative QFT. The method is based on calculating the fixed-flows of the theory, which are special RG trajectories where couplings flow to zero in the UV with the slowest possible rate allowed by RG evolution. The fixed-flows are determined by solving an algebraic system of equations, with no need to tackle the full differential equations describing the RG. This allows for a simple implementation of the method, even in models with many coupling constants. The second step of the procedure is the computation of the eigenvalues of the matrix $M$ in eq. (3.5), which determine whether the fixed-flow is UV attractive or repulsive.

UV-repulsive fixed-flows correspond to RG trajectories with isolated asymptotic behaviours, therefore singling out special values of some combination of coupling constants in the IR. For this reason, one could regard the requirement of sitting on these special RG trajectories either (pessimistically) as an extreme fine-tuning of parameters or (optimistically) as a novel way of predicting physical quantities. Our point of view is that this requirement provides a genuine prediction of the theory. The UV-repulsive fixed-flow is disconnected from the other RG trajectories in the asymptotic region and any small deformation leads to an ill-defined theory in the UV. As an analogy, take the case of a Yang-Mills theory. One cannot regard the relation between the cubic and quartic gluon couplings as a fine tuning, because such relation follows from a consistency condition of the theory in the UV.

The existence of UV-repulsive fixed-flows is essential for TAF. This is because, in practice, TAF conditions for quartic couplings can often be satisfied only if some Yukawa couplings lie on UV-repulsive fixed-flows. Moreover, stability of the scalar potential often favours quartic couplings $\lambda$ on UV-repulsive fixed-flows rather than on UV-attractive ones, because $\lambda$ is generally larger on the former than on the latter. As a result, requiring the theory to be TAF usually leads to some precise predictions of certain coupling constants in the IR. These predictions are robust against deformations in the UV, since they correspond to IR-attractive solutions. In particular, gravitational or super-weak interactions could modify the exact locations of the fixed-flows, but would not change the numerical values of the IR predictions.

We exemplified our technique in the case of the SM, studying under which conditions it satisfies TAF. We found that this happens for $g_{1}=0, M_{t}=186 \mathrm{GeV}, M_{\tau}=0, M_{h}=$ $163 \mathrm{GeV}$ (or $M_{h}<163 \mathrm{GeV}$ if unstable vacua are permitted). Since these conditions 
are unphysical (although not too far from reality), we searched for TAF extensions of the SM at the weak scale. The simplest candidates are based on the Pati-Salam group $\mathrm{SU}(2)_{L} \otimes \mathrm{SU}(2)_{R} \otimes \mathrm{SU}(4)_{\mathrm{PS}}$, for which we found some explicit examples, and on the trinification group $\mathrm{SU}(3)_{L} \otimes \mathrm{SU}(3)_{R} \otimes \mathrm{SU}(3)_{c}$, for which we have not found a fully realistic model. Our technique has been proven useful to perform systematic searches for TAF theories and determine their IR predictions.

\section{Acknowledgments}

This project started thanks to discussions with A. Arvanitaki, S. Dimopoulos, S. Dubovski, and G. Villadoro. We thank K. Kannike, R. Rattazzi, W. Skiba and M. Strassler for useful discussions. This work was supported by the ESF grant MTT8. The work of Alberto Salvio has been also supported by the Spanish Ministry of Economy and Competitiveness under grant FPA2012-32828, Consolider-CPAN (CSD2007-00042), the grant SEV-20120249 of the "Centro de Excelencia Severo Ochoa" Programme and the grant HEPHACOSS2009/ESP1473 from the C.A. de Madrid.

\section{A RGE for Pati-Salam models}

We give here the one-loop RGE for a wide class of Pati-Salam models containing the scalars $\phi_{L}, \phi_{R}, \phi, \Sigma$ and the fermions $\psi, \psi_{L}, \psi_{R}, \psi_{1}, \psi_{\Sigma}, Q_{L}, Q_{R}$ defined in section 6.1. While this model does not allow for TAF solutions, the RGE for Yukawa and quartic couplings of the TAF models proposed in section 6.2 are found by dropping all interactions involving the scalar $\phi_{L}$. The RGE for the gauge couplings depend on the chosen field content, see e.g. eq. (6.8).

The Yukawa couplings for one generation of fermions are

$$
\begin{aligned}
-\mathscr{L}_{Y}= & y \psi_{R} \psi_{L} \phi+y_{c} \psi_{R} \psi_{L} \phi^{c}+y_{N} \psi_{L} \psi \phi_{R}+y_{E} \psi \psi_{R} \phi_{L}+y_{R Q} \psi_{R} Q_{L} \phi_{R}+y_{\psi} \psi_{\Sigma}^{2} \Sigma \\
& +y_{L Q} \psi_{L} Q_{R} \phi_{L}+y_{R} \psi_{\Sigma} \psi_{R} \phi_{R}^{*}+y_{L} \psi_{\Sigma} \psi_{L} \phi_{L}^{*}+y_{\Sigma} Q_{L} Q_{R} \Sigma+y_{\nu} \psi_{1} \psi_{R} \phi_{R}^{*}+\text { h.c. }
\end{aligned}
$$

The RGE for the Yukawa couplings are

$$
\begin{aligned}
& (4 \pi)^{2} \frac{d y}{d \ln \mu}=y\left(20 y_{c}^{2}+2 y_{E}^{2}-\frac{9 g_{L}^{2}}{4}-\frac{9 g_{R}^{2}}{4}-\frac{45 g_{4}^{2}}{4}+\frac{15 y_{L}^{2}}{8}\right. \\
& \left.+\frac{5 y_{L Q}^{2}}{2}+2 y_{N}^{2}+\frac{15 y_{R}^{2}}{8}+\frac{5 y_{R Q}^{2}}{2}+y_{\nu}^{2}\right)+12 y^{3} \\
& (4 \pi)^{2} \frac{d y_{c}}{d \ln \mu}=y_{c}\left(2 y_{E}^{2}-\frac{9 g_{L}^{2}}{4}-\frac{9 g_{R}^{2}}{4}-\frac{45 g_{4}^{2}}{4}+\frac{15 y_{L}^{2}}{8}+\frac{5 y_{L Q}^{2}}{2}+2 y_{N}^{2}\right. \\
& \left.+\frac{15 y_{R}^{2}}{8}+\frac{5 y_{R Q}^{2}}{2}+20 y^{2}+y_{\nu}^{2}\right)+12 y_{c}^{3} \\
& (4 \pi)^{2} \frac{d y_{R}}{d \ln \mu}=y_{R}\left(2 y_{c}^{2}+2 y_{E}^{2}-\frac{9 g_{R}^{2}}{4}-\frac{141 g_{4}^{2}}{8}+y_{L}^{2}+4 y_{N}^{2}+\frac{11 y_{R Q}^{2}}{2}+2 y^{2}+3 y_{\nu}^{2}+\frac{3 y_{\psi}^{2}}{8}\right) \\
& +8 y_{E} y_{L} y_{N}+\frac{53 y_{R}^{3}}{8}
\end{aligned}
$$




$$
\begin{aligned}
(4 \pi)^{2} \frac{d y_{L}}{d \ln \mu}= & y_{L}\left(2 y_{c}^{2}+4 y_{E}^{2}-\frac{9 g_{L}^{2}}{4}-\frac{141 g_{4}^{2}}{8}+\frac{11 y_{L Q}^{2}}{2}+2 y_{N}^{2}+y_{R}^{2}+2 y^{2}+\frac{3 y_{\psi}^{2}}{8}\right) \\
& +8 y_{E} y_{N} y_{R}+\frac{53 y_{L}^{3}}{8} \\
(4 \pi)^{2} \frac{d y_{R Q}}{d \ln \mu}= & y_{R Q}\left(2 y_{c}^{2}+2 y_{E}^{2}-\frac{9 g_{R}^{2}}{4}-\frac{153 g_{4}^{2}}{8}+4 y_{N}^{2}\right. \\
& \left.+\frac{33 y_{R}^{2}}{8}+2 y^{2}-y_{\nu}^{2}+\frac{9 y_{\Sigma}^{2}}{16}\right)+\frac{19 y_{R Q}^{3}}{2} \\
(4 \pi)^{2} \frac{d y_{L Q}}{d \ln \mu}= & y_{L Q}\left(2 y_{c}^{2}+4 y_{E}^{2}-\frac{9 g_{L}^{2}}{4}-\frac{153 g_{4}^{2}}{8}+\frac{33 y_{L}^{2}}{8}+2 y_{N}^{2}+2 y^{2}+\frac{9 y_{\Sigma}^{2}}{16}\right)+\frac{19 y_{L Q}^{3}}{2} \\
(4 \pi)^{2} \frac{d y_{E}}{d \ln \mu}= & y_{E}\left(2 y_{c}^{2}-\frac{9 g_{L}^{2}}{4}-\frac{9 g_{R}^{2}}{2}-\frac{45 g_{4}^{2}}{8}+\frac{15 y_{L}^{2}}{4}+5 y_{L Q}^{2}+4 y_{N}^{2}+\frac{15 y_{R}^{2}}{8}\right. \\
& \left.+\frac{5 y_{R Q}^{2}}{2}+2 y^{2}+y_{\nu}^{2}\right)+10 y_{E}^{3}+\frac{15}{2} y_{L} y_{N} y_{R} \\
(4 \pi)^{2} \frac{d y_{N}}{d \ln \mu}= & y_{N}\left(2 y_{c}^{2}+4 y_{E}^{2}-\frac{9 g_{L}^{2}}{2}-\frac{9 g_{R}^{2}}{4}-\frac{45 g_{4}^{2}}{8}+\frac{15 y_{L}^{2}}{8}+\frac{5 y_{L Q}^{2}}{2}\right. \\
& \left.+\frac{15 y_{R}^{2}}{4}+5 y_{R Q}^{2}+2 y^{2}+2 y_{\nu}^{2}\right)+\frac{15}{2} y_{E} y_{L} y_{R}+10 y_{N}^{3} \\
(4 \pi)^{2} \frac{d y_{\nu}}{d \ln \mu}= & y_{\nu}\left(2 y_{c}^{2}+2 y_{E}^{2}-\frac{9 g_{R}^{2}}{4}-\frac{45 g_{4}^{2}}{8}+4 y_{N}^{2}+\frac{45 y_{R}^{2}}{8}-\frac{5 y_{R Q}^{2}}{2}+2 y^{2}\right)+11 y_{\nu}^{3} \\
(4 \pi)^{2} \frac{d y_{\psi}}{d \ln \mu}= & y_{\psi}\left(-24 g_{4}^{2}+2 y_{L}^{2}+2 y_{R}^{2}+\frac{3 y_{\Sigma}^{2}}{2}\right)+\frac{7 y_{\psi}^{3}}{4} \\
(4 \pi)^{2} \frac{d y_{\Sigma}}{d \ln \mu}= & y_{\Sigma}\left(-27 g_{4}^{2}+2 y_{L Q}^{2}+2 y_{R Q}^{2}+\frac{3 y_{\psi}^{2}}{4}\right)+\frac{31 y_{\Sigma}^{3}}{8} \\
& =(A)
\end{aligned}
$$

The most general quartic potential is

$$
\begin{aligned}
V\left(\phi, \phi_{R}, \phi_{L}, \Sigma\right)= & V_{\phi_{R}}+V_{\phi_{L}}+V_{\phi}+V_{\Sigma}+V_{\phi \phi_{L}} \\
& +V_{\phi \phi_{R}}+V_{\phi_{L} \phi_{R}}+V_{\phi_{L} \phi_{R}}^{B}+V_{\phi \Sigma}+V_{\phi_{R} \Sigma}+V_{\phi_{L} \Sigma}+V_{X}
\end{aligned}
$$

where

$$
\begin{aligned}
V_{\phi_{R}}= & \lambda_{R 1} \operatorname{Tr}^{2}\left(\phi_{R} \phi_{R}^{\dagger}\right)+\lambda_{R 2} \operatorname{Tr}\left(\phi_{R} \phi_{R}^{\dagger} \phi_{R} \phi_{R}^{\dagger}\right), \\
V_{\phi_{L}}= & \lambda_{L 1} \operatorname{Tr}^{2}\left(\phi_{L}^{\dagger} \phi_{L}\right)+\lambda_{L 2} \operatorname{Tr}\left(\phi_{L}^{\dagger} \phi_{L} \phi_{L}^{\dagger} \phi_{L}\right), \\
V_{\phi}= & \lambda_{1} \operatorname{Tr}^{2}\left(\phi^{\dagger} \phi\right)+\operatorname{Re} \lambda_{2} \operatorname{Tr}^{2}\left(\phi^{\dagger} \phi^{c}\right)+ \\
& +\operatorname{Re} \lambda_{3} \operatorname{Tr}\left(\phi^{\dagger} \phi\right) \operatorname{Tr}\left(\phi^{\dagger} \phi^{c}\right)+\left(\lambda_{4}-2 \operatorname{Re} \lambda_{2}\right)\left|\operatorname{Tr}\left(\phi^{\dagger} \phi^{c}\right)\right|^{2}, \\
V_{\phi \phi_{R}}= & \lambda_{R \phi 1} \operatorname{Tr}\left(\phi_{R} \phi_{R}^{\dagger}\right) \operatorname{Tr}\left(\phi^{\dagger} \phi\right)+\operatorname{Re} \lambda_{R \phi 2} \operatorname{Tr}\left(\phi_{R} \phi_{R}^{\dagger}\right) \operatorname{Tr}\left(\phi^{\dagger} \phi^{c}\right) \\
& +\lambda_{R \phi 3} \operatorname{Tr}\left(\phi_{R} \phi_{R}^{\dagger} \phi^{\dagger} \phi\right) \\
V_{\phi \phi_{L}}= & \lambda_{L \phi 1} \operatorname{Tr}\left(\phi_{L}^{\dagger} \phi_{L}\right) \operatorname{Tr}\left(\phi^{\dagger} \phi\right)+\operatorname{Re} \lambda_{L \phi 2} \operatorname{Tr}\left(\phi_{L}^{\dagger} \phi_{L}\right) \operatorname{Tr}\left(\phi^{\dagger} \phi^{c}\right) \\
& +\lambda_{L \phi 3} \operatorname{Tr}\left(\phi_{L}^{\dagger} \phi_{L} \phi \phi^{\dagger}\right), \\
V_{\phi_{L} \phi_{R}}= & \lambda_{L R 1} \operatorname{Tr}\left(\phi_{L}^{\dagger} \phi_{L}\right) \operatorname{Tr}\left(\phi_{R} \phi_{R}^{\dagger}\right)+\lambda_{L R 2} \operatorname{Tr}\left(\phi_{L} \phi_{L}^{\dagger} \phi_{R}^{\dagger} \phi_{R}\right) \\
& +\operatorname{Re} \lambda_{L R 3} \operatorname{Tr}\left[\left(\phi_{R} \phi_{L}\right)\left(\phi_{R} \phi_{L}\right)^{c}\right],
\end{aligned}
$$




$$
\begin{aligned}
V_{\phi_{L} \phi_{R}}^{B} & =\operatorname{Re} \lambda_{B} \epsilon_{I J K L} \epsilon_{i j} \epsilon_{k \ell} \phi_{L I i} \phi_{L J j} \phi_{R K k}^{*} \phi_{R L \ell}^{*}, \\
V_{\Sigma} & =\lambda_{\Sigma 1} \operatorname{Tr}^{2}\left(\Sigma^{2}\right)+\lambda_{\Sigma 2} \operatorname{Tr}\left(\Sigma^{4}\right), \\
V_{\phi \Sigma} & =\lambda_{\Sigma \phi 1} \operatorname{Tr}\left(\Sigma^{2}\right) \operatorname{Tr}\left(\phi^{\dagger} \phi\right)+\operatorname{Re} \lambda_{\Sigma \phi 2} \operatorname{Tr}\left(\Sigma^{2}\right) \operatorname{Tr}\left(\phi^{\dagger} \phi^{c}\right), \\
V_{\phi_{R} \Sigma} & =\lambda_{\Sigma \phi_{R} 1} \operatorname{Tr}\left(\Sigma^{2}\right) \operatorname{Tr}\left(\phi_{R}^{\dagger} \phi_{R}\right)+\lambda_{\Sigma \phi_{R} 2} \operatorname{Tr}\left(\phi_{R}^{\dagger} \phi_{R} \Sigma^{2}\right), \\
V_{\phi_{L} \Sigma} & =\lambda_{\Sigma \phi_{L} 1} \operatorname{Tr}\left(\Sigma^{2}\right) \operatorname{Tr}\left(\phi_{L}^{\dagger} \phi_{L}\right)+\lambda_{\Sigma \phi_{L} 2} \operatorname{Tr}\left(\phi_{L} \phi_{L}^{\dagger} \Sigma^{2}\right), \\
V_{X} & =\operatorname{Re} \lambda_{X 1} \operatorname{Tr}\left(\phi_{L} \phi_{L R} \phi_{R} \Sigma\right)+\operatorname{Re} \lambda_{X 2} \operatorname{Tr}\left(\phi_{L} \phi_{L R}^{c} \phi_{R} \Sigma\right) .
\end{aligned}
$$

The symbol Re (real part) precedes quartic interactions that can support complex couplings $\lambda_{i}$. With an abuse of notation, in the RGE listed below we denote as $\lambda_{i}$ the real part and as $\theta_{i}$ the imaginary part of complex couplings $\lambda_{i}$. The RGE for the quartic couplings are

$$
\begin{aligned}
& (4 \pi)^{2} \frac{d \lambda_{\Sigma \phi_{L} 1}}{d \ln \mu}=\lambda_{\Sigma \phi_{L} 1}\left(8 y_{E}^{2}-\frac{9 g_{L}^{2}}{2}-\frac{141 g_{4}^{2}}{4}+34 \lambda_{\Sigma 1}+\frac{29 \lambda_{\Sigma 2}}{2}+\frac{15 y_{L}^{2}}{2}+144 \lambda_{L 1}+96 \lambda_{L 2}\right. \\
& \left.+10 y_{L Q}^{2}+3 y_{\Sigma}^{2}+\frac{3 y_{\psi}^{2}}{2}\right)+\frac{3 g_{4}^{4}}{2}-\frac{5}{8} y_{L}^{2} y_{\psi}^{2}+32 \lambda_{L 1} \lambda_{\Sigma \phi_{L} 2}+16 \lambda_{L 2} \lambda_{\Sigma \phi_{L} 2} \\
& -\frac{1}{2} y_{L Q}^{2} y_{\Sigma}^{2}+64 \lambda_{L R 1} \lambda_{\Sigma \phi_{R} 1}+16 \lambda_{L R 1} \lambda_{\Sigma \phi_{R} 2}+16 \lambda_{L R 2} \lambda_{\Sigma \phi_{R} 1}+8 \lambda_{\Sigma \phi_{L} 1}^{2} \\
& +\frac{15 \lambda_{\Sigma 1} \lambda_{\Sigma \phi_{L} 2}}{2}+\frac{19 \lambda_{\Sigma 2} \lambda_{\Sigma \phi_{L} 2}}{8}+2 \lambda_{\Sigma \phi_{L} 2}^{2}+32 \lambda_{\Sigma \phi 1} \lambda_{\phi_{L} \phi 1}+128 \theta_{\Sigma \phi 2} \theta_{\phi_{L} \phi 2} \\
& +128 \lambda_{\Sigma \phi 2} \lambda_{\phi_{L} \phi 2}+16 \lambda_{\Sigma \phi 1} \lambda_{\phi_{L} \phi 3} \\
& (4 \pi)^{2} \frac{d \lambda_{\Sigma \phi_{R} 1}}{d \ln \mu}=\lambda_{\Sigma \phi_{R} 1}\left(-\frac{9 g_{R}^{2}}{2}-\frac{141 g_{4}^{2}}{4}+34 \lambda_{\Sigma 1}+\frac{29 \lambda_{\Sigma 2}}{2}+8 y_{N}^{2}+\frac{15 y_{R}^{2}}{2}+144 \lambda_{R 1}\right. \\
& \left.+96 \lambda_{R 2}+10 y_{R Q}^{2}+4 y_{\nu}^{2}+3 y_{\Sigma}^{2}+\frac{3 y_{\psi}^{2}}{2}\right)+\frac{3 g_{4}^{4}}{2}+64 \lambda_{L R 1} \lambda_{\Sigma \phi_{L} 1} \\
& +16 \lambda_{L R 1} \lambda_{\Sigma \phi_{L} 2}+16 \lambda_{L R 2} \lambda_{\Sigma \phi_{L} 1}-\frac{5}{8} y_{R}^{2} y_{\psi}^{2}+32 \lambda_{R 1} \lambda_{\Sigma \phi_{R} 2}+16 \lambda_{R 2} \lambda_{\Sigma \phi_{R} 2} \\
& -\frac{1}{2} y_{R Q}^{2} y_{\Sigma}^{2}+8 \lambda_{\Sigma \phi_{R} 1}^{2}+\frac{15 \lambda_{\Sigma 1} \lambda_{\Sigma \phi_{R} 2}}{2}+\frac{19 \lambda_{\Sigma 2} \lambda_{\Sigma \phi_{R} 2}}{8}+2 \lambda_{\Sigma \phi_{R} 2}^{2} \\
& +32 \lambda_{\Sigma \phi 1} \lambda_{\phi_{R} \phi 1}+128 \theta_{\Sigma \phi 2} \theta_{\phi_{R} \phi 2}+128 \lambda_{\Sigma \phi 2} \lambda_{\phi_{R} \phi 2}+16 \lambda_{\Sigma \phi 1} \lambda_{\phi_{R} \phi 3} \\
& (4 \pi)^{2} \frac{d \lambda_{\Sigma \phi 1}}{d \ln \mu}=\lambda_{\Sigma \phi 1}\left(16 y_{c}^{2}-\frac{9 g_{L}^{2}}{2}-\frac{9 g_{R}^{2}}{2}-24 g_{4}^{2}+34 \lambda_{\Sigma 1}+\frac{29 \lambda_{\Sigma 2}}{2}+80 \lambda_{1}-64 \lambda_{2}+32 \lambda_{4}\right. \\
& \left.+16 y^{2}+3 y_{\Sigma}^{2}+\frac{3 y_{\psi}^{2}}{2}\right)+32 \theta_{\Sigma \phi 2}^{2}+192 \theta_{3} \theta_{\Sigma \phi 2}+8 \lambda_{\Sigma \phi 1}^{2}+32 \lambda_{\Sigma \phi 2}^{2} \\
& +192 \lambda_{3} \lambda_{\Sigma \phi 2}+64 \lambda_{\Sigma \phi_{L} 1} \lambda_{\phi_{L} \phi 1}+32 \lambda_{\Sigma \phi_{L} 1} \lambda_{\phi_{L} \phi 3}+16 \lambda_{\Sigma \phi_{L} 2} \lambda_{\phi_{L} \phi 1} \\
& +8 \lambda_{\Sigma \phi_{L} 2} \lambda_{\phi_{L} \phi 3}+64 \lambda_{\Sigma \phi_{R} 1} \lambda_{\phi_{R} \phi 1}+32 \lambda_{\Sigma \phi_{R} 1} \lambda_{\phi_{R} \phi 3}+16 \lambda_{\Sigma \phi_{R} 2} \lambda_{\phi_{R} \phi 1} \\
& +8 \lambda_{\Sigma \phi_{R} 2} \lambda_{\phi_{R} \phi 3}+8 \theta_{X 1}^{2}+8 \lambda_{X 1}^{2}+8 \theta_{X 2}^{2}+8 \lambda_{X 2}^{2} \\
& (4 \pi)^{2} \frac{d \lambda_{\Sigma \phi_{L} 2}}{d \ln \mu}=\lambda_{\Sigma \phi_{L} 2}\left(8 y_{E}^{2}-\frac{9 g_{L}^{2}}{2}-\frac{141 g_{4}^{2}}{4}+4 \lambda_{\Sigma 1}+5 \lambda_{\Sigma 2}+\frac{15 y_{L}^{2}}{2}+16 \lambda_{L 1}+32 \lambda_{L 2}\right.
\end{aligned}
$$




$$
\begin{aligned}
& \left.+10 y_{L Q}^{2}+16 \lambda_{\Sigma \phi_{L} 1}+3 y_{\Sigma}^{2}+\frac{3 y_{\psi}^{2}}{2}\right)+6 g_{4}^{4}-\frac{1}{2} y_{L}^{2} y_{\psi}^{2}-4 y_{L Q}^{2} y_{\Sigma}^{2} \\
& +16 \lambda_{L R 2} \lambda_{\Sigma \phi_{R} 2}+6 \lambda_{\Sigma \phi_{L} 2}^{2}+16 \theta_{X 1}^{2}+16 \lambda_{X 1}^{2}+16 \theta_{X 2}^{2}+16 \lambda_{X 2}^{2} \\
& (4 \pi)^{2} \frac{d \lambda_{\Sigma \phi_{R} 2}}{d \ln \mu}=\lambda_{\Sigma \phi_{R} 2}\left(-\frac{9 g_{R}^{2}}{2}-\frac{141 g_{4}^{2}}{4}+4 \lambda_{\Sigma 1}+5 \lambda_{\Sigma 2}+8 y_{N}^{2}+\frac{15 y_{R}^{2}}{2}+16 \lambda_{R 1}\right. \\
& \left.+32 \lambda_{R 2}+10 y_{R Q}^{2}+16 \lambda_{\Sigma \phi_{R} 1}+4 y_{\nu}^{2}+3 y_{\Sigma}^{2}+\frac{3 y_{\psi}^{2}}{2}\right)+6 g_{4}^{4}+16 \lambda_{L R 2} \lambda_{\Sigma \phi_{L} 2} \\
& -\frac{1}{2} y_{R}^{2} y_{\psi}^{2}-4 y_{R Q}^{2} y_{\Sigma}^{2}+6 \lambda_{\Sigma \phi_{R} 2}^{2}+16 \theta_{X 1}^{2}+16 \lambda_{X 1}^{2}+16 \theta_{X 2}^{2}+16 \lambda_{X 2}^{2} \\
& (4 \pi)^{2} \frac{d \lambda_{\Sigma \phi 2}}{d \ln \mu}=\lambda_{\Sigma \phi 2}\left(16 y_{c}^{2}-\frac{9 g_{L}^{2}}{2}-\frac{9 g_{R}^{2}}{2}-24 g_{4}^{2}+34 \lambda_{\Sigma 1}+\frac{29 \lambda_{\Sigma 2}}{2}+16 \lambda_{\Sigma \phi 1}+16 \lambda_{1}\right. \\
& \left.+64 \lambda_{2}+64 \lambda_{4}+16 y^{2}+3 y_{\Sigma}^{2}+\frac{3 y_{\psi}^{2}}{2}\right)+192 \theta_{2} \theta_{\Sigma \phi 2}+48 \lambda_{3} \lambda_{\Sigma \phi 1} \\
& +64 \lambda_{\Sigma \phi_{L} 1} \lambda_{\phi_{L} \phi 2}+16 \lambda_{\Sigma \phi_{L} 2} \lambda_{\phi_{L} \phi 2}+64 \lambda_{\Sigma \phi_{R} 1} \lambda_{\phi_{R} \phi 2}+16 \lambda_{\Sigma \phi_{R} 2} \lambda_{\phi_{R} \phi 2} \\
& +8 \theta_{X 1} \theta_{X 2}+8 \lambda_{X 1} \lambda_{X 2} \\
& (4 \pi)^{2} \frac{d \theta_{\Sigma \phi 2}}{d \ln \mu}=\theta_{\Sigma \phi 2}\left(16 y_{c}^{2}-\frac{9 g_{L}^{2}}{2}-\frac{9 g_{R}^{2}}{2}-24 g_{4}^{2}+34 \lambda_{\Sigma 1}+\frac{29 \lambda_{\Sigma 2}}{2}+16 \lambda_{\Sigma \phi 1}+16 \lambda_{1}\right. \\
& \left.-320 \lambda_{2}+64 \lambda_{4}+16 y^{2}+3 y_{\Sigma}^{2}+\frac{3 y_{\psi}^{2}}{2}\right)+48 \theta_{3} \lambda_{\Sigma \phi 1}+192 \theta_{2} \lambda_{\Sigma \phi 2} \\
& +64 \lambda_{\Sigma \phi_{L} 1} \theta_{\phi_{L} \phi 2}+16 \lambda_{\Sigma \phi_{L} 2} \theta_{\phi_{L} \phi 2}+64 \lambda_{\Sigma \phi_{R} 1} \theta_{\phi_{R} \phi 2}+16 \lambda_{\Sigma \phi_{R} 2} \theta_{\phi_{R} \phi 2} \\
& +8 \lambda_{X 1} \theta_{X 2}-8 \theta_{X 1} \lambda_{X 2} \\
& (4 \pi)^{2} \frac{d \lambda_{B}}{d \ln \mu}=\lambda_{B}\left(8 y_{E}^{2}-\frac{9 g_{L}^{2}}{2}-\frac{9 g_{R}^{2}}{2}-\frac{45 g_{4}^{2}}{2}+\frac{15 y_{L}^{2}}{2}+16 \lambda_{L 1}-16 \lambda_{L 2}+10 y_{L Q}^{2}\right. \\
& \left.+32 \lambda_{L R 1}-32 \lambda_{L R 2}+8 y_{N}^{2}+\frac{15 y_{R}^{2}}{2}+16 \lambda_{R 1}-16 \lambda_{R 2}+10 y_{R Q}^{2}+4 y_{\nu}^{2}\right) \\
& (4 \pi)^{2} \frac{d \theta_{B}}{d \ln \mu}=\theta_{B}\left(8 y_{E}^{2}-\frac{9 g_{L}^{2}}{2}-\frac{9 g_{R}^{2}}{2}-\frac{45 g_{4}^{2}}{2}+\frac{15 y_{L}^{2}}{2}+16 \lambda_{L 1}-16 \lambda_{L 2}+10 y_{L Q}^{2}\right. \\
& \left.+32 \lambda_{L R 1}-32 \lambda_{L R 2}+8 y_{N}^{2}+\frac{15 y_{R}^{2}}{2}+16 \lambda_{R 1}-16 \lambda_{R 2}+10 y_{R Q}^{2}+4 y_{\nu}^{2}\right) \\
& (4 \pi)^{2} \frac{d \lambda_{L 1}}{d \ln \mu}=128 \theta_{B}^{2}+128 \lambda_{B}^{2}+\lambda_{L 1}\left(16 y_{E}^{2}-9 g_{L}^{2}-\frac{45 g_{4}^{2}}{2}+15 y_{L}^{2}+192 \lambda_{L 2}+20 y_{L Q}^{2}\right) \\
& +\frac{27}{32} g_{4}^{2} g_{L}^{2}+\frac{9 g_{L}^{4}}{32}+\frac{27 g_{4}^{4}}{128}-5 y_{L}^{2} y_{L Q}^{2}-\frac{y_{L}^{4}}{32}+192 \lambda_{L 1}^{2}+48 \lambda_{L 2}^{2}-\frac{y_{L Q}^{4}}{2} \\
& +32 \lambda_{L R 1}^{2}+16 \lambda_{L R 1} \lambda_{L R 2}+32 \theta_{L R 3}^{2}+32 \lambda_{L R 3}^{2}+\frac{15 \lambda_{\Sigma \phi_{L} 1}^{2}}{2} \\
& +\frac{15 \lambda_{\Sigma \phi_{L} 1} \lambda_{\Sigma \phi_{L} 2}}{4}+\frac{9 \lambda_{\Sigma \phi_{L} 2}^{2}}{32}+16 \lambda_{\phi_{L} \phi 1}^{2}+16 \lambda_{\phi_{L} \phi 1} \lambda_{\phi_{L} \phi 3} \\
& +64 \theta_{\phi_{L} \phi 2}^{2}+64 \lambda_{\phi_{L} \phi 2}^{2}
\end{aligned}
$$




$$
\begin{aligned}
(4 \pi)^{2} \frac{d \lambda_{L 2}}{d \ln \mu}= & -128 \theta_{B}^{2}-128 \lambda_{B}^{2}+\lambda_{L 2}\left(16 y_{E}^{2}-9 g_{L}^{2}-\frac{45 g_{4}^{2}}{2}+15 y_{L}^{2}+96 \lambda_{L 1}+20 y_{L Q}^{2}\right) \\
& -4 y_{E}^{4}-\frac{9}{16} g_{4}^{2} g_{L}^{2}+\frac{9 g_{4}^{4}}{16}+5 y_{L}^{2} y_{L Q}^{2}-\frac{7 y_{L}^{4}}{4}+96 \lambda_{L 2}^{2}-3 y_{L Q}^{4}+8 \lambda_{L R 2}^{2} \\
& -32 \theta_{L R 3}^{2}-32 \lambda_{L R 3}^{2}+\frac{3 \lambda_{\Sigma \phi_{L} 2}^{2}}{4}+8 \lambda_{\phi_{L} \phi 3}^{2} \\
(4 \pi)^{2} \frac{d \lambda_{R 1}}{d \ln \mu}= & 128 \theta_{B}^{2}+128 \lambda_{B}^{2}+\lambda_{R 1}\left(-9 g_{R}^{2}-\frac{45 g_{4}^{2}}{2}+16 y_{N}^{2}+15 y_{R}^{2}+192 \lambda_{R 2}\right. \\
& \left.+20 y_{R Q}^{2}+8 y_{\nu}^{2}\right)+\frac{27}{32} g_{4}^{2} g_{R}^{2}+\frac{9 g_{R}^{4}}{32}+\frac{27 g_{4}^{4}}{128}+32 \lambda_{L R 1}^{2}+16 \lambda_{L R 1} \lambda_{L R 2} \\
& +32 \theta_{L R 3}^{2}+32 \lambda_{L R 3}^{2}-5 y_{R}^{2} y_{R Q}^{2}+\frac{1}{2} y_{R}^{2} y_{\nu}^{2}-\frac{y_{R}^{4}}{32}+192 \lambda_{R 1}^{2}+48 \lambda_{R 2}^{2}-\frac{y_{R Q}^{4}}{2} \\
& +\frac{15 \lambda_{\Sigma \phi_{R} 1}^{2}}{2}+\frac{15 \lambda_{\Sigma \phi_{R} 1} \lambda_{\Sigma \phi_{R} 2}}{4}+\frac{9 \lambda_{\Sigma \phi_{R} 2}^{2}}{32}+16 \lambda_{\phi_{R} \phi 1}^{2}+16 \lambda_{\phi_{R} \phi 1} \lambda_{\phi_{R} \phi 3} \\
& +64 \theta_{\phi_{R} \phi 2}^{2}+64 \lambda_{\phi_{R} \phi 2}^{2}-2 y_{\nu}^{4}
\end{aligned}
$$$$
(4 \pi)^{2} \frac{d \lambda_{R 2}}{d \ln \mu}=-128 \theta_{B}^{2}-128 \lambda_{B}^{2}+\lambda_{R 2}\left(-9 g_{R}^{2}-\frac{45 g_{4}^{2}}{2}+16 y_{N}^{2}+15 y_{R}^{2}+96 \lambda_{R 1}\right.
$$$$
\left.+20 y_{R Q}^{2}+8 y_{\nu}^{2}\right)-\frac{9}{16} g_{4}^{2} g_{R}^{2}+\frac{9 g_{4}^{4}}{16}+8 \lambda_{L R 2}^{2}-32 \theta_{L R 3}^{2}-32 \lambda_{L R 3}^{2}-4 y_{N}^{4}
$$$$
+5 y_{R}^{2} y_{R Q}^{2}-2 y_{R}^{2} y_{\nu}^{2}-\frac{7 y_{R}^{4}}{4}+96 \lambda_{R 2}^{2}-3 y_{R Q}^{4}+\frac{3 \lambda_{\Sigma \phi_{R} 2}^{2}}{4}+8 \lambda_{\phi_{R} \phi 3}^{2}
$$$$
(4 \pi)^{2} \frac{d \lambda_{X 1}}{d \ln \mu}=\lambda_{X 1}\left(8 y_{c}^{2}+4 y_{E}^{2}-\frac{9 g_{L}^{2}}{2}-\frac{9 g_{R}^{2}}{2}-\frac{93 g_{4}^{2}}{4}+4 \lambda_{\Sigma \phi 1}+\frac{15 y_{L}^{2}}{4}+5 y_{L Q}^{2}+8 \lambda_{L R 1}\right.
$$$$
+4 y_{N}^{2}+\frac{15 y_{R}^{2}}{4}+5 y_{R Q}^{2}+4 \lambda_{\Sigma \phi_{L} 1}+7 \lambda_{\Sigma \phi_{L} 2}+4 \lambda_{\Sigma \phi_{R} 1}+7 \lambda_{\Sigma \phi_{R} 2}+8 \lambda_{\phi_{L} \phi 1}
$$$$
\left.+16 \lambda_{\phi_{L} \phi 3}+8 \lambda_{\phi_{R} \phi 1}+16 \lambda_{\phi_{R} \phi 3}+8 y^{2}+2 y_{\nu}^{2}+\frac{3 y_{\Sigma}^{2}}{2}+\frac{3 y_{\psi}^{2}}{4}\right)-6 y_{c} y_{L Q} y_{R Q} y_{\Sigma}
$$$$
-3 y y_{L} y_{R} y_{\psi}-16 \theta_{L R 3} \theta_{X 2}-16 \lambda_{L R 3} \lambda_{X 2}+16 \theta_{\phi_{L} \phi 2} \theta_{X 2}+16 \lambda_{\phi_{L} \phi 2} \lambda_{X 2}
$$$$
+16 \theta_{\phi_{R} \phi 2} \theta_{X 2}+16 \lambda_{\phi_{R} \phi 2} \lambda_{X 2}+8 \theta_{\Sigma \phi 2} \theta_{X 2}+8 \lambda_{\Sigma \phi 2} \lambda_{X 2}
$$$$
(4 \pi)^{2} \frac{d \theta_{X 1}}{d \ln \mu}=\theta_{X 1}\left(8 y_{c}^{2}+4 y_{E}^{2}-\frac{9 g_{L}^{2}}{2}-\frac{9 g_{R}^{2}}{2}-\frac{93 g_{4}^{2}}{4}+4 \lambda_{\Sigma \phi 1}+\frac{15 y_{L}^{2}}{4}+5 y_{L Q}^{2}+8 \lambda_{L R 1}\right.
$$$$
+4 y_{N}^{2}+\frac{15 y_{R}^{2}}{4}+5 y_{R Q}^{2}+4 \lambda_{\Sigma \phi_{L} 1}+7 \lambda_{\Sigma \phi_{L} 2}+4 \lambda_{\Sigma \phi_{R} 1}+7 \lambda_{\Sigma \phi_{R} 2}+8 \lambda_{\phi_{L} \phi 1}
$$$$
\left.+16 \lambda_{\phi_{L} \phi 3}+8 \lambda_{\phi_{R} \phi 1}+16 \lambda_{\phi_{R} \phi 3}+8 y^{2}+2 y_{\nu}^{2}+\frac{3 y_{\Sigma}^{2}}{2}+\frac{3 y_{\psi}^{2}}{4}\right)+16 \lambda_{L R 3} \theta_{X 2}
$$$$
-16 \theta_{L R 3} \lambda_{X 2}+16 \lambda_{\phi_{L} \phi 2} \theta_{X 2}-16 \theta_{\phi_{L} \phi 2} \lambda_{X 2}+16 \lambda_{\phi_{R} \phi 2} \theta_{X 2}-16 \theta_{\phi_{R} \phi 2} \lambda_{X 2}
$$$$
-8 \theta_{\Sigma \phi 2} \lambda_{X 2}+8 \lambda_{\Sigma \phi 2} \theta_{X 2}
$$

$(4 \pi)^{2} \frac{d \lambda_{X 2}}{d \ln \mu}=\lambda_{X 2}\left(8 y_{c}^{2}+4 y_{E}^{2}-\frac{9 g_{L}^{2}}{2}-\frac{9 g_{R}^{2}}{2}-\frac{93 g_{4}^{2}}{4}+4 \lambda_{\Sigma \phi 1}+\frac{15 y_{L}^{2}}{4}+5 y_{L Q}^{2}+8 \lambda_{L R 1}\right.$ 


$$
\begin{aligned}
& +4 y_{N}^{2}+\frac{15 y_{R}^{2}}{4}+5 y_{R Q}^{2}+4 \lambda_{\Sigma \phi_{L} 1}+7 \lambda_{\Sigma \phi_{L} 2}+4 \lambda_{\Sigma \phi_{R} 1}+7 \lambda_{\Sigma \phi_{R} 2}+8 \lambda_{\phi_{L} \phi 1} \\
& \left.-8 \lambda_{\phi_{L} \phi 3}+8 \lambda_{\phi_{R} \phi 1}-8 \lambda_{\phi_{R} \phi 3}+8 y^{2}+2 y_{\nu}^{2}+\frac{3 y_{\Sigma}^{2}}{2}+\frac{3 y_{\psi}^{2}}{4}\right) \\
& -3 y_{c} y_{L} y_{R} y_{\psi}-6 y y_{L Q} y_{R Q} y_{\Sigma}-16 \theta_{L R 3} \theta_{X 1}-16 \lambda_{L R 3} \lambda_{X 1}-16 \theta_{\phi_{L} \phi 2} \theta_{X 1} \\
& +16 \lambda_{\phi_{L} \phi 2} \lambda_{X 1}-16 \theta_{\phi_{R} \phi 2} \theta_{X 1}+16 \lambda_{\phi_{R} \phi 2} \lambda_{X 1}-8 \theta_{\Sigma \phi 2} \theta_{X 1}+8 \lambda_{\Sigma \phi 2} \lambda_{X 1} \\
& (4 \pi)^{2} \frac{d \theta_{X 2}}{d \ln \mu}=\theta_{X 2}\left(8 y_{c}^{2}+4 y_{E}^{2}-\frac{9 g_{L}^{2}}{2}-\frac{9 g_{R}^{2}}{2}-\frac{93 g_{4}^{2}}{4}+4 \lambda_{\Sigma \phi 1}+\frac{15 y_{L}^{2}}{4}+5 y_{L Q}^{2}+8 \lambda_{L R 1}\right. \\
& +4 y_{N}^{2}+\frac{15 y_{R}^{2}}{4}+5 y_{R Q}^{2}+4 \lambda_{\Sigma \phi_{L} 1}+7 \lambda_{\Sigma \phi_{L} 2}+4 \lambda_{\Sigma \phi_{R} 1}+7 \lambda_{\Sigma \phi_{R} 2}+8 \lambda_{\phi_{L} \phi 1} \\
& \left.-8 \lambda_{\phi_{L} \phi 3}+8 \lambda_{\phi_{R} \phi 1}-8 \lambda_{\phi_{R} \phi 3}+8 y^{2}+2 y_{\nu}^{2}+\frac{3 y_{\Sigma}^{2}}{2}+\frac{3 y_{\psi}^{2}}{4}\right) \\
& +16 \lambda_{L R 3} \theta_{X 1}-16 \theta_{L R 3} \lambda_{X 1}+16 \lambda_{\phi_{L} \phi 2} \theta_{X 1}+16 \theta_{\phi_{L} \phi 2} \lambda_{X 1}+16 \lambda_{\phi_{R} \phi 2} \theta_{X 1} \\
& +16 \theta_{\phi_{R} \phi 2} \lambda_{X 1}+8 \theta_{\Sigma \phi 2} \lambda_{X 1}+8 \lambda_{\Sigma \phi 2} \theta_{X 1} \\
& (4 \pi)^{2} \frac{d \lambda_{\Sigma 1}}{d \ln \mu}=\lambda_{\Sigma 1}\left(-48 g_{4}^{2}+29 \lambda_{\Sigma 2}+6 y_{\Sigma}^{2}+3 y_{\psi}^{2}\right)+9 g_{4}^{4}+64 \theta_{\Sigma \phi 2}^{2}+46 \lambda_{\Sigma 1}^{2}+\frac{57 \lambda_{\Sigma 2}^{2}}{8} \\
& +16 \lambda_{\Sigma \phi 1}^{2}+64 \lambda_{\Sigma \phi 2}^{2}+32 \lambda_{\Sigma \phi_{L} 1}^{2}+16 \lambda_{\Sigma \phi_{L} 1} \lambda_{\Sigma \phi_{L} 2}+32 \lambda_{\Sigma \phi_{R} 1}^{2} \\
& +16 \lambda_{\Sigma \phi_{R} 1} \lambda_{\Sigma \phi_{R} 2}-\frac{3 y_{\Sigma}^{4}}{8}-\frac{y_{\psi}^{4}}{2} \\
& (4 \pi)^{2} \frac{d \lambda_{\Sigma 2}}{d \ln \mu}=\lambda_{\Sigma 2}\left(-48 g_{4}^{2}+24 \lambda_{\Sigma 1}+6 y_{\Sigma}^{2}+3 y_{\psi}^{2}\right)+12 g_{4}^{4}+7 \lambda_{\Sigma 2}^{2}+8 \lambda_{\Sigma \phi_{L} 2}^{2} \\
& +8 \lambda_{\Sigma \phi_{R} 2}^{2}-\frac{3 y_{\Sigma}^{4}}{2}+\frac{y_{\psi}^{4}}{2} \\
& (4 \pi)^{2} \frac{d \lambda_{1}}{d \ln \mu}=\lambda_{1}\left(32 y_{c}^{2}-9 g_{L}^{2}-9 g_{R}^{2}-128 \lambda_{2}+64 \lambda_{4}+32 y^{2}\right)-8 y_{c}^{4}+\frac{3}{16} g_{L}^{2} g_{R}^{2} \\
& +\frac{9 g_{L}^{4}}{32}+\frac{9 g_{R}^{4}}{32}+256 \theta_{2}^{2}+192 \theta_{3}^{2}+\frac{15 \lambda_{\Sigma \phi 1}^{2}}{2}+128 \lambda_{1}^{2}+512 \lambda_{2}^{2}+192 \lambda_{3}^{2} \\
& +64 \lambda_{4}^{2}-256 \lambda_{2} \lambda_{4}+32 \lambda_{\phi_{L} \phi 1}^{2}+32 \lambda_{\phi_{L} \phi 1} \lambda_{\phi_{L} \phi 3}+16 \lambda_{\phi_{L} \phi 3}^{2}+32 \lambda_{\phi_{R} \phi 1}^{2} \\
& +32 \lambda_{\phi_{R} \phi 1} \lambda_{\phi_{R} \phi 3}+16 \lambda_{\phi_{R} \phi 3}^{2}-8 y^{4} \\
& (4 \pi)^{2} \frac{d \lambda_{2}}{d \ln \mu}=\lambda_{2}\left(32 y_{c}^{2}-9 g_{L}^{2}-9 g_{R}^{2}+96 \lambda_{1}+192 \lambda_{4}+32 y^{2}\right)-4 y^{2} y_{c}^{2}-\frac{15 \theta_{\Sigma \phi 2}^{2}}{2}-48 \theta_{3}^{2} \\
& +\frac{15 \lambda_{\Sigma \phi 2}^{2}}{2}-384 \lambda_{2}^{2}+48 \lambda_{3}^{2}-32 \theta_{\phi_{L} \phi 2}^{2}+32 \lambda_{\phi_{L} \phi 2}^{2}-32 \theta_{\phi_{R} \phi 2}^{2}+32 \lambda_{\phi_{R} \phi 2}^{2} \\
& (4 \pi)^{2} \frac{d \theta_{2}}{d \ln \mu}=\theta_{2}\left(32 y_{c}^{2}-9 g_{L}^{2}-9 g_{R}^{2}+96 \lambda_{1}-384 \lambda_{2}+192 \lambda_{4}+32 y^{2}\right)+15 \theta_{\Sigma \phi 2} \lambda_{\Sigma \phi 2} \\
& +96 \theta_{3} \lambda_{3}+64 \theta_{\phi_{L} \phi 2} \lambda_{\phi_{L} \phi 2}+64 \theta_{\phi_{R} \phi 2} \lambda_{\phi_{R} \phi 2}
\end{aligned}
$$




$$
\begin{aligned}
& (4 \pi)^{2} \frac{d \lambda_{3}}{d \ln \mu}=\lambda_{3}\left(32 y_{c}^{2}-9 g_{L}^{2}-9 g_{R}^{2}+192 \lambda_{1}+192 \lambda_{4}+32 y^{2}\right)-8 y^{3} y_{c}-8 y y_{c}^{3} \\
& +384 \theta_{2} \theta_{3}+15 \lambda_{\Sigma \phi 1} \lambda_{\Sigma \phi 2}+64 \lambda_{\phi_{L} \phi 1} \lambda_{\phi_{L} \phi 2}+32 \lambda_{\phi_{L} \phi 2} \lambda_{\phi_{L} \phi 3} \\
& +64 \lambda_{\phi_{R} \phi 1} \lambda_{\phi_{R} \phi 2}+32 \lambda_{\phi_{R} \phi 2} \lambda_{\phi_{R} \phi 3} \\
& (4 \pi)^{2} \frac{d \theta_{3}}{d \ln \mu}=\theta_{3}\left(32 y_{c}^{2}-9 g_{L}^{2}-9 g_{R}^{2}+192 \lambda_{1}-768 \lambda_{2}+192 \lambda_{4}+32 y^{2}\right)+15 \theta_{\Sigma \phi 2} \lambda_{\Sigma \phi 1} \\
& +384 \theta_{2} \lambda_{3}+64 \lambda_{\phi_{L} \phi 1} \theta_{\phi_{L} \phi 2}+32 \theta_{\phi_{L} \phi 2} \lambda_{\phi_{L} \phi 3}+64 \lambda_{\phi_{R} \phi 1} \theta_{\phi_{R} \phi 2}+32 \theta_{\phi_{R} \phi 2} \lambda_{\phi_{R} \phi 3} \\
& (4 \pi)^{2} \frac{d \lambda_{4}}{d \ln \mu}=\lambda_{4}\left(32 y_{c}^{2}-9 g_{L}^{2}-9 g_{R}^{2}+96 \lambda_{1}+128 \lambda_{2}+32 y^{2}\right)-24 y^{2} y_{c}^{2}+4 y_{c}^{4} \\
& +\frac{3}{8} g_{L}^{2} g_{R}^{2}+512 \theta_{2}^{2}+30 \lambda_{\Sigma \phi 2}^{2}+192 \lambda_{3}^{2}+64 \lambda_{4}^{2}+128 \lambda_{\phi_{L} \phi 2}^{2} \\
& -8 \lambda_{\phi_{L} \phi 3}^{2}+128 \lambda_{\phi_{R} \phi 2}^{2}-8 \lambda_{\phi_{R} \phi 3}^{2}+4 y^{4} \\
& (4 \pi)^{2} \frac{d \lambda_{\phi_{L} \phi 1}}{d \ln \mu}=\lambda_{\phi_{L} \phi 1}\left(16 y_{c}^{2}+8 y_{E}^{2}-9 g_{L}^{2}-\frac{9 g_{R}^{2}}{2}-\frac{45 g_{4}^{2}}{4}+80 \lambda_{1}-64 \lambda_{2}+32 \lambda_{4}+\frac{15 y_{L}^{2}}{2}\right. \\
& \left.+144 \lambda_{L 1}+96 \lambda_{L 2}+10 y_{L Q}^{2}+16 y^{2}\right)-4 y_{c}^{2} y_{E}^{2}-\frac{15}{2} y_{c}^{2} y_{L}^{2}-4 y^{2} y_{E}^{2}+\frac{9 g_{L}^{4}}{16} \\
& +64 \lambda_{L 1} \lambda_{\phi_{L} \phi 3}+16 \lambda_{L 2} \lambda_{\phi_{L} \phi 3}-10 y^{2} y_{L Q}^{2}+64 \lambda_{L R 1} \lambda_{\phi_{R} \phi 1}+32 \lambda_{L R 1} \lambda_{\phi_{R} \phi 3} \\
& +16 \lambda_{L R 2} \lambda_{\phi_{R} \phi 1}+8 \lambda_{L R 2} \lambda_{\phi_{R} \phi 3}+15 \lambda_{\Sigma \phi 1} \lambda_{\Sigma \phi_{L} 1}+\frac{15 \lambda_{\Sigma \phi 1} \lambda_{\Sigma \phi_{L} 2}}{4} \\
& +16 \lambda_{\phi_{L} \phi 1}^{2}+64 \theta_{\phi_{L} \phi 2}^{2}+192 \theta_{3} \theta_{\phi_{L} \phi 2}+64 \lambda_{\phi_{L} \phi 2}^{2}+192 \lambda_{3} \lambda_{\phi_{L} \phi 2}+8 \lambda_{\phi_{L} \phi 3}^{2} \\
& +32 \lambda_{1} \lambda_{\phi_{L} \phi 3}-64 \lambda_{2} \lambda_{\phi_{L} \phi 3}+32 \lambda_{4} \lambda_{\phi_{L} \phi 3}+\frac{15 \theta_{X 2}^{2}}{2}+\frac{15 \lambda_{X 2}^{2}}{2} \\
& (4 \pi)^{2} \frac{d \lambda_{\phi_{L} \phi 2}}{d \ln \mu}=\lambda_{\phi_{L} \phi 2}\left(16 y_{c}^{2}+8 y_{E}^{2}-9 g_{L}^{2}-\frac{9 g_{R}^{2}}{2}-\frac{45 g_{4}^{2}}{4}+16 \lambda_{1}+64 \lambda_{2}+64 \lambda_{4}+\frac{15 y_{L}^{2}}{2}\right. \\
& \left.+144 \lambda_{L 1}+96 \lambda_{L 2}+10 y_{L Q}^{2}+32 \lambda_{\phi_{L} \phi 1}+16 \lambda_{\phi_{L} \phi 3}+16 y^{2}\right)-4 y y_{c} y_{E}^{2} \\
& -\frac{15}{4} y y_{c} y_{L}^{2}-5 y y_{c} y_{L Q}^{2}+64 \lambda_{L R 1} \lambda_{\phi_{R} \phi 2}+16 \lambda_{L R 2} \lambda_{\phi_{R} \phi 2}+15 \lambda_{\Sigma \phi 2} \lambda_{\Sigma \phi_{L} 1} \\
& +\frac{15 \lambda_{\Sigma \phi 2} \lambda_{\Sigma \phi_{L} 2}}{4}+48 \lambda_{3} \lambda_{\phi_{L} \phi 1}+192 \theta_{2} \theta_{\phi_{L} \phi 2}+24 \lambda_{3} \lambda_{\phi_{L} \phi 3} \\
& +\frac{15 \theta_{X 1} \theta_{X 2}}{4}+\frac{15 \lambda_{X 1} \lambda_{X 2}}{4} \\
& (4 \pi)^{2} \frac{d \theta_{\phi_{L} \phi 2}}{d \ln \mu}=\theta_{\phi_{L} \phi 2}\left(16 y_{c}^{2}+8 y_{E}^{2}-9 g_{L}^{2}-\frac{9 g_{R}^{2}}{2}-\frac{45 g_{4}^{2}}{4}+16 \lambda_{1}-320 \lambda_{2}+64 \lambda_{4}+\frac{15 y_{L}^{2}}{2}\right. \\
& \left.+144 \lambda_{L 1}+96 \lambda_{L 2}+10 y_{L Q}^{2}+32 \lambda_{\phi_{L} \phi 1}+16 \lambda_{\phi_{L} \phi 3}+16 y^{2}\right) \\
& +64 \lambda_{L R 1} \theta_{\phi_{R} \phi 2}+16 \lambda_{L R 2} \theta_{\phi_{R} \phi 2}+15 \theta_{\Sigma \phi 2} \lambda_{\Sigma \phi_{L} 1}+\frac{15 \theta_{\Sigma \phi 2} \lambda_{\Sigma \phi_{L} 2}}{4} \\
& +48 \theta_{3} \lambda_{\phi_{L} \phi 1}+192 \theta_{2} \lambda_{\phi_{L} \phi 2}+24 \theta_{3} \lambda_{\phi_{L} \phi 3}+\frac{15 \lambda_{X 1} \theta_{X 2}}{4}-\frac{15 \theta_{X 1} \lambda_{X 2}}{4}
\end{aligned}
$$




$$
\begin{aligned}
(4 \pi)^{2} \frac{d \lambda_{\phi_{L} \phi 3}}{d \ln \mu}= & \lambda_{\phi_{L} \phi 3}\left(16 y_{c}^{2}+8 y_{E}^{2}-9 g_{L}^{2}-\frac{9 g_{R}^{2}}{2}-\frac{45 g_{4}^{2}}{4}+16 \lambda_{1}+64 \lambda_{2}-32 \lambda_{4}+\frac{15 y_{L}^{2}}{2}\right. \\
& \left.+16 \lambda_{L 1}+64 \lambda_{L 2}+10 y_{L Q}^{2}+32 \lambda_{\phi_{L} \phi 1}+16 y^{2}\right)+\frac{15}{2} y_{c}^{2} y_{L}^{2}-10 y_{c}^{2} y_{L Q}^{2} \\
& -\frac{15}{2} y^{2} y_{L}^{2}+10 y^{2} y_{L Q}^{2}+16 \lambda_{\phi_{L} \phi 3}^{2}+\frac{15 \theta_{X 1}^{2}}{2}+\frac{15 \lambda_{X 1}^{2}}{2}-\frac{15 \theta_{X 2}^{2}}{2}-\frac{15 \lambda_{X 2}^{2}}{2} \\
(4 \pi)^{2} \frac{d \lambda_{L R 1}}{d \ln \mu}= & \lambda_{L R 1}\left(8 y_{E}^{2}-\frac{9 g_{L}^{2}}{2}-\frac{9 g_{R}^{2}}{2}-\frac{45 g_{4}^{2}}{2}+\frac{15 y_{L}^{2}}{2}+144 \lambda_{L 1}+96 \lambda_{L 2}+10 y_{L Q}^{2}\right. \\
& \left.+8 y_{N}^{2}+\frac{15 y_{R}^{2}}{2}+144 \lambda_{R 1}+96 \lambda_{R 2}+10 y_{R Q}^{2}+4 y_{\nu}^{2}\right)+256 \theta_{B}^{2}+256 \lambda_{B}^{2} \\
& -4 y_{E} y_{L} y_{N} y_{R}-4 y_{E}^{2} y_{N}^{2}-2 y_{E}^{2} y_{R}^{2}-2 y_{E}^{2} y_{R Q}^{2}+\frac{27 g_{4}^{4}}{64}-2 y_{L}^{2} y_{N}^{2}-\frac{17}{16} y_{L}^{2} y_{R}^{2} \\
& +32 \lambda_{L 1} \lambda_{L R 2}+16 \lambda_{L 2} \lambda_{L R 2}-2 y_{L Q}^{2} y_{N}^{2}+16 \lambda_{L R 1}^{2}+8 \lambda_{L R 2}^{2}+32 \lambda_{L R 2} \lambda_{R 1} \\
& +16 \lambda_{L R 2} \lambda_{R 2}+32 \theta_{L R 3}^{2}+32 \lambda_{L R 3}^{2}+15 \lambda_{\Sigma \phi_{L} 1} \lambda_{\Sigma \phi_{R} 1}+\frac{15 \lambda_{\Sigma \phi_{L} 1} \lambda_{\Sigma \phi_{R} 2}}{4} \\
& +\frac{15 \lambda_{\Sigma \phi_{L} 2} \lambda_{\Sigma \phi_{R} 1}}{4}+\frac{9 \lambda_{\Sigma \phi_{L} 2} \lambda_{\Sigma \phi_{R} 2}}{16}+32 \lambda_{\phi_{L} \phi 1} \lambda_{\phi_{R} \phi 1}+16 \lambda_{\phi_{L} \phi 1} \lambda_{\phi_{R} \phi 3} \\
& +128 \theta_{\phi_{L} \phi 2} \theta_{\phi_{R} \phi 2}+128 \lambda_{\phi_{L} \phi 2} \lambda_{\phi_{R} \phi 2}+16 \lambda_{\phi_{L} \phi 3} \lambda_{\phi_{R} \phi 1}+8 \lambda_{\phi_{L} \phi 3} \lambda_{\phi_{R} \phi 3} \\
& +2 \theta_{X 1}^{2}+2 \lambda_{X 1}^{2}+2 \theta_{X 2}^{2}+2 \lambda_{X 2}^{2}
\end{aligned}
$$$$
(4 \pi)^{2} \frac{d \lambda_{L R 2}}{d \ln \mu}=\lambda_{L R 2}\left(8 y_{E}^{2}-\frac{9 g_{L}^{2}}{2}-\frac{9 g_{R}^{2}}{2}-\frac{45 g_{4}^{2}}{2}+\frac{15 y_{L}^{2}}{2}+16 \lambda_{L 1}+32 \lambda_{L 2}+10 y_{L Q}^{2}\right.
$$$$
\left.+32 \lambda_{L R 1}+8 y_{N}^{2}+\frac{15 y_{R}^{2}}{2}+16 \lambda_{R 1}+32 \lambda_{R 2}+10 y_{R Q}^{2}+4 y_{\nu}^{2}\right)-256 \theta_{B}^{2}
$$$$
-256 \lambda_{B}^{2}+y_{E} y_{L} y_{N} y_{R}+\frac{1}{2} y_{E}^{2} y_{R}^{2}-2 y_{E}^{2} y_{R Q}^{2}-4 y_{E}^{2} y_{\nu}^{2}+\frac{9 g_{4}^{4}}{8}+\frac{1}{2} y_{L}^{2} y_{N}^{2}
$$$$
+\frac{1}{2} y_{L}^{2} y_{R}^{2}-2 y_{L Q}^{2} y_{N}^{2}+32 \lambda_{L R 2}^{2}+64 \theta_{L R 3}^{2}+64 \lambda_{L R 3}^{2}+\frac{3 \lambda_{\Sigma \phi_{L} 2} \lambda_{\Sigma \phi_{R} 2}}{2}
$$$$
-\frac{\theta_{X 1}^{2}}{2}-\frac{\lambda_{X 1}^{2}}{2}-\frac{\theta_{X 2}^{2}}{2}-\frac{\lambda_{X 2}^{2}}{2}
$$$$
(4 \pi)^{2} \frac{d \lambda_{L R 3}}{d \ln \mu}=\lambda_{L R 3}\left(8 y_{E}^{2}-\frac{9 g_{L}^{2}}{2}-\frac{9 g_{R}^{2}}{2}-\frac{45 g_{4}^{2}}{2}+\frac{15 y_{L}^{2}}{2}+16 \lambda_{L 1}-16 \lambda_{L 2}+10 y_{L Q}^{2}\right.
$$$$
\left.+32 \lambda_{L R 1}+48 \lambda_{L R 2}+8 y_{N}^{2}+\frac{15 y_{R}^{2}}{2}+16 \lambda_{R 1}-16 \lambda_{R 2}+10 y_{R Q}^{2}+4 y_{\nu}^{2}\right)
$$$$
+\frac{5 \theta_{X 1} \theta_{X 2}}{2}-\frac{5 \lambda_{X 1} \lambda_{X 2}}{2}
$$$$
(4 \pi)^{2} \frac{d \theta_{L R 3}}{d \ln \mu}=\theta_{L R 3}\left(8 y_{E}^{2}-\frac{9 g_{L}^{2}}{2}-\frac{9 g_{R}^{2}}{2}-\frac{45 g_{4}^{2}}{2}+\frac{15 y_{L}^{2}}{2}+16 \lambda_{L 1}-16 \lambda_{L 2}+10 y_{L Q}^{2}\right.
$$$$
\left.+32 \lambda_{L R 1}++48 \lambda_{L R 2}+8 y_{N}^{2}+\frac{15 y_{R}^{2}}{2}+16 \lambda_{R 1}-16 \lambda_{R 2}+10 y_{R Q}^{2}+4 y_{\nu}^{2}\right)
$$$$
-\frac{5 \lambda_{X 1} \theta_{X 2}}{2}-\frac{5 \theta_{X 1} \lambda_{X 2}}{2}
$$ 


$$
\begin{aligned}
& (4 \pi)^{2} \frac{d \lambda_{\phi_{R} \phi 1}}{d \ln \mu}=\lambda_{\phi_{R} \phi 1}\left(16 y_{c}^{2}-\frac{9 g_{L}^{2}}{2}-9 g_{R}^{2}-\frac{45 g_{4}^{2}}{4}+80 \lambda_{1}-64 \lambda_{2}+32 \lambda_{4}+8 y_{N}^{2}+\frac{15 y_{R}^{2}}{2}\right. \\
& \left.+144 \lambda_{R 1}+96 \lambda_{R 2}+10 y_{R Q}^{2}+16 y^{2}+4 y_{\nu}^{2}\right)-4 y_{c}^{2} y_{N}^{2}-\frac{15}{2} y_{c}^{2} y_{R}^{2}-4 y_{c}^{2} y_{\nu}^{2} \\
& +\frac{9 g_{R}^{4}}{16}+64 \lambda_{L R 1} \lambda_{\phi_{L} \phi 1}+32 \lambda_{L R 1} \lambda_{\phi_{L} \phi 3}+16 \lambda_{L R 2} \lambda_{\phi_{L} \phi 1}+8 \lambda_{L R 2} \lambda_{\phi_{L} \phi 3} \\
& -4 y^{2} y_{N}^{2}+64 \lambda_{R 1} \lambda_{\phi_{R} \phi 3}+16 \lambda_{R 2} \lambda_{\phi_{R} \phi 3}-10 y^{2} y_{R Q}^{2}+15 \lambda_{\Sigma \phi 1} \lambda_{\Sigma \phi_{R} 1} \\
& +\frac{15 \lambda_{\Sigma \phi 1} \lambda_{\Sigma \phi_{R} 2}}{4}+16 \lambda_{\phi_{R} \phi 1}^{2}+64 \theta_{\phi_{R} \phi 2}^{2}+192 \theta_{3} \theta_{\phi_{R} \phi 2}+64 \lambda_{\phi_{R} \phi 2}^{2} \\
& +192 \lambda_{3} \lambda_{\phi_{R} \phi 2}+8 \lambda_{\phi_{R} \phi 3}^{2}+32 \lambda_{1} \lambda_{\phi_{R} \phi 3}-64 \lambda_{2} \lambda_{\phi_{R} \phi 3}+32 \lambda_{4} \lambda_{\phi_{R} \phi 3} \\
& +\frac{15 \theta_{X 2}^{2}}{2}+\frac{15 \lambda_{X 2}^{2}}{2} \\
& (4 \pi)^{2} \frac{d \lambda_{\phi_{R} \phi 2}}{d \ln \mu}=\lambda_{\phi_{R} \phi 2}\left(16 y_{c}^{2}-\frac{9 g_{L}^{2}}{2}-9 g_{R}^{2}-\frac{45 g_{4}^{2}}{4}+16 \lambda_{1}+64 \lambda_{2}+64 \lambda_{4}+8 y_{N}^{2}+\frac{15 y_{R}^{2}}{2}\right. \\
& \left.+144 \lambda_{R 1}+96 \lambda_{R 2}+10 y_{R Q}^{2}+32 \lambda_{\phi_{R} \phi 1}++16 \lambda_{\phi_{R} \phi 3}+16 y^{2}+4 y_{\nu}^{2}\right) \\
& -4 y y_{c} y_{N}^{2}-\frac{15}{4} y y_{c} y_{R}^{2}-5 y y_{c} y_{R Q}^{2}-2 y y_{c} y_{\nu}^{2}+64 \lambda_{L R 1} \lambda_{\phi_{L} \phi 2}+16 \lambda_{L R 2} \lambda_{\phi_{L} \phi 2} \\
& +15 \lambda_{\Sigma \phi 2} \lambda_{\Sigma \phi_{R} 1}+\frac{15 \lambda_{\Sigma \phi 2} \lambda_{\Sigma \phi_{R} 2}}{4}+48 \lambda_{3} \lambda_{\phi_{R} \phi 1}+192 \theta_{2} \theta_{\phi_{R} \phi 2}+24 \lambda_{3} \lambda_{\phi_{R} \phi 3} \\
& +\frac{15 \theta_{X 1} \theta_{X 2}}{4}+\frac{15 \lambda_{X 1} \lambda_{X 2}}{4} \\
& (4 \pi)^{2} \frac{d \theta_{\phi_{R} \phi 2}}{d \ln \mu}=\theta_{\phi_{R} \phi 2}\left(16 y_{c}^{2}-\frac{9 g_{L}^{2}}{2}-9 g_{R}^{2}-\frac{45 g_{4}^{2}}{4}+16 \lambda_{1}-320 \lambda_{2}+64 \lambda_{4}+8 y_{N}^{2}+\frac{15 y_{R}^{2}}{2}\right. \\
& \left.+144 \lambda_{R 1}+96 \lambda_{R 2}+10 y_{R Q}^{2}+32 \lambda_{\phi_{R} \phi 1}+16 \lambda_{\phi_{R} \phi 3}+16 y^{2}+4 y_{\nu}^{2}\right) \\
& +64 \lambda_{L R 1} \theta_{\phi_{L} \phi 2}+16 \lambda_{L R 2} \theta_{\phi_{L} \phi 2}+15 \theta_{\Sigma \phi 2} \lambda_{\Sigma \phi_{R} 1}+\frac{15 \theta_{\Sigma \phi 2} \lambda_{\Sigma \phi_{R} 2}}{4}+48 \theta_{3} \lambda_{\phi_{R} \phi 1} \\
& +192 \theta_{2} \lambda_{\phi_{R} \phi 2}+24 \theta_{3} \lambda_{\phi_{R} \phi 3}+\frac{15 \lambda_{X 1} \theta_{X 2}}{4}-\frac{15 \theta_{X 1} \lambda_{X 2}}{4} \\
& (4 \pi)^{2} \frac{d \lambda_{\phi_{R} \phi 3}}{d \ln \mu}=\lambda_{\phi_{R} \phi 3}\left(16 y_{c}^{2}-\frac{9 g_{L}^{2}}{2}-9 g_{R}^{2}-\frac{45 g_{4}^{2}}{4}+16 \lambda_{1}+64 \lambda_{2}-32 \lambda_{4}+8 y_{N}^{2}+\frac{15 y_{R}^{2}}{2}\right. \\
& \left.+16 \lambda_{R 1}+64 \lambda_{R 2}+10 y_{R Q}^{2}+32 \lambda_{\phi_{R} \phi 1}+16 y^{2}+4 y_{\nu}^{2}\right)+\frac{15}{2} y_{c}^{2} y_{R}^{2}-10 y_{c}^{2} y_{R Q}^{2} \\
& +4 y_{c}^{2} y_{\nu}^{2}-\frac{15}{2} y^{2} y_{R}^{2}+10 y^{2} y_{R Q}^{2}+16 \lambda_{\phi_{R} \phi 3}^{2}+\frac{15 \theta_{X 1}^{2}}{2}+\frac{15 \lambda_{X 1}^{2}}{2} \\
& -\frac{15 \theta_{X 2}^{2}}{2}-\frac{15 \lambda_{X 2}^{2}}{2}-4 y^{2} y_{\nu}^{2}
\end{aligned}
$$

\section{B Flavour bounds on Pati-Salam vector leptoquarks}

In this section we discuss the experimental bounds on the massive vector leptoquarks $W_{\mu}^{\prime}$, of electric charge $\pm 2 / 3$, coming from $\mathrm{SU}(4)_{\mathrm{PS}} / \mathrm{SU}(3)_{c}$. These vector bosons are coupled 
to the left-handed current $\bar{q}_{L} \gamma^{\mu} \ell_{L}$ only in the Pati-Salam models of ref. [82], where $q_{L}$ and $\ell_{L}$ are unified in $\psi_{L}$ [see eq. (6.5))], but not in the models of ref. [81] where $q_{L}$ is in $\psi_{L}$ and $\ell_{L}$ in $\psi$ [see eq. (6.4)]. On the other hand, the right-handed current interaction is present in both frameworks and is given by

$$
\mathscr{L}_{W^{\prime}}=\frac{g_{3}}{\sqrt{2}} W_{i a} \bar{d}_{R}^{i} \gamma_{\mu} e_{R}^{a} W_{\mu}^{\prime}+\text { h.c. }
$$

using Dirac notation for the fermions. Here $i$ and $a$ are flavour indices and $W_{i a}$ is a new unitary matrix that describes the right-handed misalignment between the effective downquark and charged-lepton Yukawa couplings. Integrating out the heavy $W^{\prime}$ fields leads to the following effective interaction

$$
\mathscr{L}_{\text {eff }}^{\text {de }}=-\frac{g_{3}^{2}}{2 m_{W^{\prime}}^{2}} W_{i a} W_{b j}^{\dagger}\left(\bar{d}_{R}^{i} \gamma_{\mu} e_{R}^{a}\right)\left(\bar{e}_{R}^{b} \gamma_{\mu} d_{R}^{j}\right)+\text { h.c. }
$$

The bounds on these effective operators (expressed as bounds on $M_{W^{\prime}}$ always assuming the maximal possible mixing $W_{i a} W_{b j}^{\dagger}=1$ ) from various LFV and FCNC processes are reported in table $5 .{ }^{16}$ In order to deduce from this table the lowest allowed value for $M_{W^{\prime}}$ we need to determine the structure of the right-handed quark-lepton mixing matrix $W_{i a}$. To this purpose, we distinguish two cases.

The first case corresponds to the Yukawa interaction in eq. (6.4), where we have two completely independent Yukawa couplings for down quarks and charged leptons. As a result, $W_{i a}$ is arbitrary and we can tune it in order to minimize the bounds on $M_{W^{\prime}}$ given in table 5. The two most severe bounds (on the $d d e \mu$ and $s d e \mu$ flavour structures) force us to consider a mixing matrix of the type

$$
W=\left(\begin{array}{ccc}
0 & 0 & 1 \\
s_{s e} & c_{s e} & 0 \\
-c_{s e} & s_{s e} & 0
\end{array}\right)
$$

to forbid any $d \leftrightarrow e$ and $d \leftrightarrow \mu$ mixing. All bounds from processes with flavour conservation either in the quark (rows 1-4 in table 5) or in the lepton sector (rows 5-8 in table 5) disappear if we assume that $W_{i a}$ is a permutation matrix. As a result, the constraint on $M_{W^{\prime}}$ is minimised for $s_{s e}=0$ and it is set by $\operatorname{BR}\left(\tau \rightarrow K^{*} \mu\right)$ :

$$
M_{W^{\prime}}>8.8 \mathrm{TeV} \text {. }
$$

The second case corresponds to the Yukawa interaction in eq. (6.5) with $Q_{L} \oplus Q_{R}$ pairs. Now the structure of $W_{i a}$ is not arbitrary: the effective down-type and charged-lepton Yukawa couplings are necessarily quasi aligned. The resulting $W_{i a}$ is close to a diagonal matrix, with a mixing angle in the $1-2$ sector of the order of the Cabibbo angle. In this case we cannot avoid the bound on $M_{W^{\prime}}$ in the range of $100-200 \mathrm{TeV}$ dictated by the dde $\mu$ and $s d e \mu$ entries in table 5 . The situation is even worse due to the simultaneous presence of both $d_{R}-e_{R}-W^{\prime}$ and $u_{L}-\nu_{L}-W^{\prime}$ interactions. The combination of left-handed and righthanded currents leads to an effective interaction that breaks lepton chirality, without being proportional to the corresponding lepton mass. This gives rise to an effective breaking of lepton universality in $\Gamma(\pi \rightarrow e \nu) / \Gamma(\pi \rightarrow \mu \nu)$ that implies $M_{W^{\prime}}>250 \mathrm{TeV}$ [83].

\footnotetext{
${ }^{16}$ For a previous version of this table see [84].
} 


\begin{tabular}{|l|l|c|}
\hline Flavour & Experimental constraint & Bound on $M_{W^{\prime}} \mathrm{in} \mathrm{TeV}$ \\
\hline$d d e \mu$ & $\sigma(\mu \mathrm{Ti} \rightarrow e \mathrm{Ti}) / \sigma_{0}(\mu \mathrm{Ti})<4.3 \times 10^{-12}$ & 120 \\
$s s e \mu$ & $\sigma(\mu \mathrm{Ti} \rightarrow e \mathrm{Ti}) / \sigma_{0}(\mu \mathrm{Ti})<4.3 \times 10^{-12}$ & $12 \times \sqrt{P_{s \bar{s}} / 1 \%}$ \\
$d d e \tau$ & $\mathrm{BR}\left(\tau \rightarrow \pi^{0} e\right)<8.0 \times 10^{-8}$ & 3.8 \\
$d d \mu \tau$ & $\mathrm{BR}\left(\tau \rightarrow \pi^{0} \mu\right)<1.1 \times 10^{-7}$ & 3.5 \\
\hline$s d \mu \mu$ & $\mathrm{BR}\left(K_{L} \rightarrow \bar{\mu} \mu\right)_{\mathrm{SD}}<2.5 \times 10^{-9}$ & 50 \\
$s d e e$ & $\mathrm{BR}\left(K_{L} \rightarrow \bar{e} e\right)=(9.0 \pm 6.0) \times 10^{-12}$ & 13.4 \\
$b d \mu \mu$ & $\mathrm{BR}\left(B_{d} \rightarrow \bar{\mu} \mu\right)=(3.6 \pm 1.6) \times 10^{-10}$ & 12.7 \\
$b s \mu \mu$ & $\mathrm{BR}\left(B_{s} \rightarrow \bar{\mu} \mu\right)=(2.9 \pm 0.7) \times 10^{-9}$ & 10.1 \\
\hline$s d e \mu$ & $\mathrm{BR}\left(K_{L} \rightarrow \bar{e} \mu\right)<4.7 \times 10^{-12}$ & 200 \\
\hline$s d e \tau$ & $\mathrm{BR}\left(\tau \rightarrow K^{*} e\right)<3.2 \times 10^{-8}$ & 10.3 \\
$s d \mu \tau$ & $\mathrm{BR}\left(\tau \rightarrow K^{*} \mu\right)<5.9 \times 10^{-8}$ & 8.8 \\
$b s e \mu$ & $\mathrm{BR}\left(B^{+} \rightarrow K^{+} \bar{e} \mu\right)<9.1 \times 10^{-8}$ & 8.3 \\
$b d e \mu$ & $\mathrm{BR}\left(B^{+} \rightarrow \pi^{+} \bar{e} \mu\right)<1.7 \times 10^{-7}$ & 7.1 \\
$b d \mu \tau$ & $\mathrm{BR}\left(B_{d} \rightarrow \bar{\mu} \tau\right)<2.2 \times 10^{-5}$ & 3.0 \\
$b d e \tau$ & $\mathrm{BR}\left(B_{d} \rightarrow \bar{e} \tau\right)<2.8 \times 10^{-5}$ & 2.8 \\
\hline
\end{tabular}

Table 5. Bounds on the vector leptoquark mass $M_{W^{\prime}}$ assuming maximal mixing angles in each case $\left(W_{i a} W_{b j}^{\dagger}=1\right)$ from lepton-flavour-violating and flavour-changing-neutral-current processes. Here $P_{s \bar{s}}$ denotes the ratio of the strange-quark density in nuclei, normalised to that of down-type quarks $\left(P_{s \bar{s}}=\langle N|\bar{s} s| N\rangle /\langle N|\bar{d} d| N\rangle\right)$, for which we have assumed a reference value of $1 \%$ (see e.g. [85]).

\section{RGE for trinification models}

We first list the one-loop RGE for the trinification models described in section 6.3. The gauge $\beta$-function coefficients are $b_{L}=b_{R}=5-n_{H} / 2$ and $b_{3}=5$, where $n_{H}$ is the number of Higgs multiplets. The RGE for the Yukawa couplings of eq. (6.18) are

$$
\begin{aligned}
& (4 \pi)^{2} \frac{d y_{Q_{1}}}{d \ln \mu}=y_{Q_{1}}\left(-4 g_{L}^{2}-4 g_{R}^{2}-8 g_{3}^{2}+2 y_{L_{1}}^{2}+6 y_{Q_{2}}^{2}\right)+2 y_{L_{1}} y_{L_{2}} y_{Q_{2}}+6 y_{Q_{1}}^{3} \\
& (4 \pi)^{2} \frac{d y_{Q_{2}}}{d \ln \mu}=y_{Q_{2}}\left(-4 g_{L}^{2}-4 g_{R}^{2}-8 g_{3}^{2}+2 y_{L_{2}}^{2}+6 y_{Q_{1}}^{2}\right)+2 y_{L_{1}} y_{L_{2}} y_{Q_{1}}+6 y_{Q_{2}}^{3} \\
& (4 \pi)^{2} \frac{d y_{L_{1}}}{d \ln \mu}=y_{L_{1}}\left(-8 g_{L}^{2}-8 g_{R}^{2}+6 y_{L_{2}}^{2}+3 y_{Q_{1}}^{2}\right)+6 y_{L_{1}}^{3}+3 y_{L_{2}} y_{Q_{1}} y_{Q_{2}} \\
& (4 \pi)^{2} \frac{d y_{L_{2}}}{d \ln \mu}=y_{L_{2}}\left(-8 g_{L}^{2}-8 g_{R}^{2}+6 y_{L_{1}}^{2}+3 y_{Q_{2}}^{2}\right)+3 y_{L_{1}} y_{Q_{1}} y_{Q_{2}}+6 y_{L_{2}}^{3}
\end{aligned}
$$

For the model with a single Higgs multiplet, $n_{H}=1$, one needs to set $y_{Q_{1}}=y_{Q}, y_{L_{1}}=y_{L}$ and $y_{Q_{2}}=y_{L_{2}}=0$. The RGE for the two quartic couplings of the scalar potential in 
eq. (6.19) are

$$
\begin{aligned}
(4 \pi)^{2} \frac{d \lambda_{a}}{d \ln \mu}= & \lambda_{a}\left(-16 g_{L}^{2}-16 g_{R}^{2}+48 \lambda_{b}+12 y_{Q}^{2}+8 y_{L}^{2}\right) \\
& +\frac{10}{3} g_{L}^{2} g_{R}^{2}+\frac{11 g_{L}^{4}}{12}+\frac{11 g_{R}^{4}}{12}+52 \lambda_{a}^{2}+12 \lambda_{b}^{2}-2 y_{L}^{4} \\
(4 \pi)^{2} \frac{d \lambda_{b}}{d \ln \mu}= & \lambda_{b}\left(-16 g_{L}^{2}-16 g_{R}^{2}+24 \lambda_{a}+12 y_{Q}^{2}+8 y_{L}^{2}\right) \\
& -2 g_{L}^{2} g_{R}^{2}+\frac{5 g_{L}^{4}}{4}+\frac{5 g_{R}^{4}}{4}+24 \lambda_{b}^{2}-6 y_{U}^{4}-2 y_{L}^{4}
\end{aligned}
$$

For the model with two Higgs fields $H_{1}$ and $H_{2}\left(n_{H}=2\right)$, the most generic scalar potential becomes

$$
V=V_{1111}+V_{2222}+V_{1122}+V_{1222}+V_{1222}
$$

where

$$
\begin{aligned}
V_{1111}= & \lambda_{1 a} \operatorname{Tr}\left(H_{1}^{\dagger} H_{1}\right)^{2}+\lambda_{1 b} \operatorname{Tr}\left(H_{1}^{\dagger} H_{1} H_{1}^{\dagger} H_{1}\right) \\
V_{2222}= & \lambda_{2 a} \operatorname{Tr}\left(H_{2}^{\dagger} H_{2}\right)^{2}+\lambda_{2 b} \operatorname{Tr}\left(H_{2}^{\dagger} H_{2} H_{2}^{\dagger} H_{2}\right) \\
V_{1222}= & \operatorname{Re} \lambda_{3 a} \operatorname{Tr}\left(H_{1}^{\dagger} H_{2}\right) \operatorname{Tr}\left(H_{2}^{\dagger} H_{2}\right)+\operatorname{Re} \lambda_{3 b} \operatorname{Tr}\left(H_{1}^{\dagger} H_{2} H_{2}^{\dagger} H_{2}\right) \\
V_{1112}= & \operatorname{Re} \lambda_{4 a} \operatorname{Tr}\left(H_{1}^{\dagger} H_{2}\right) \operatorname{Tr}\left(H_{1}^{\dagger} H_{1}\right)+\operatorname{Re} \lambda_{4 b} \operatorname{Tr}\left(H_{1}^{\dagger} H_{1} H_{1}^{\dagger} H_{2}\right) \\
V_{1122}= & \lambda_{a} \operatorname{Tr}\left(H_{1}^{\dagger} H_{1}\right) \operatorname{Tr}\left(H_{2}^{\dagger} H_{2}\right)+\lambda_{b}\left|\operatorname{Tr}\left(H_{1}^{\dagger} H_{2}\right)\right|^{2} \\
& +\lambda_{c} \operatorname{Tr}\left(H_{1}^{\dagger} H_{1} H_{2}^{\dagger} H_{2}\right)+\lambda_{d} \operatorname{Tr}\left(H_{1} H_{1}^{\dagger} H_{2} H_{2}^{\dagger}\right) \\
& +\operatorname{Re} \lambda_{e} \operatorname{Tr}\left(H_{1}^{\dagger} H_{2}\right)^{2}+\operatorname{Re} \lambda_{f} \operatorname{Tr}\left(H_{1}^{\dagger} H_{2} H_{1}^{\dagger} H_{2}\right)
\end{aligned}
$$

The RGE for the quartics, setting for simplicity to zero those that violate $\mathrm{CP}$ and/or baryon number, are:

$$
\begin{aligned}
(4 \pi)^{2} \frac{d \lambda_{1 a}}{d \ln \mu}= & \lambda_{1 a}\left(48 \lambda_{1 b}-16 g_{L}^{2}-16 g_{R}^{2}+8 y_{L_{1}}^{2}+12 y_{Q_{1}}^{2}\right)+52 \lambda_{1 a}^{2}+12 \lambda_{1 b}^{2}+56 \lambda_{4 b}^{2} \\
& +2 \lambda_{a} \lambda_{b}+6 \lambda_{a} \lambda_{c}+6 \lambda_{a} \lambda_{d}+9 \lambda_{a}^{2}+\lambda_{b}^{2}+2 \lambda_{c} \lambda_{d}+4 \lambda_{e}^{2}+4 \lambda_{f}^{2} \\
& +\frac{10}{3} g_{L}^{2} g_{R}^{2}+\frac{11 g_{L}^{4}}{12}+\frac{11 g_{R}^{4}}{12}-2 y_{L_{1}}^{4} \\
(4 \pi)^{2} \frac{d \lambda_{1 b}}{d \ln \mu}= & \lambda_{1 b}\left(24 \lambda_{1 a}-16 g_{L}^{2}-16 g_{R}^{2}+8 y_{L_{1}}^{2}+12 y_{Q_{1}}^{2}\right)+24 \lambda_{1 b}^{2}+24 \lambda_{4 b}^{2}+2 \lambda_{b} \lambda_{c}+2 \lambda_{b} \lambda_{d} \\
& +3 \lambda_{c}^{2}+3 \lambda_{d}^{2}+8 \lambda_{e} \lambda_{f}-2 g_{L}^{2} g_{R}^{2}+\frac{5 g_{L}^{4}}{4}+\frac{5 g_{R}^{4}}{4}-2 y_{L_{1}}^{4}-6 y_{Q_{1}}^{4} \\
(4 \pi)^{2} \frac{d \lambda_{2 a}}{d \ln \mu}= & \lambda_{2 a}\left(48 \lambda_{2 b}-16 g_{L}^{2}-16 g_{R}^{2}+8 y_{L_{2}}^{2}+12 y_{Q_{2}}^{2}\right)+52 \lambda_{2 a}^{2}+24 \lambda_{2 b} \lambda_{3 a}+18 \lambda_{2 b}^{2} \\
& +26 \lambda_{3 a}^{2}+2 \lambda_{a} \lambda_{b}+6 \lambda_{a} \lambda_{c}+6 \lambda_{a} \lambda_{d}+9 \lambda_{a}^{2}+\lambda_{b}^{2}+2 \lambda_{c} \lambda_{d}+4 \lambda_{e}^{2}+4 \lambda_{f}^{2} \\
& +\frac{10}{3} g_{L}^{2} g_{R}^{2}+\frac{11 g_{L}^{4}}{12}+\frac{11 g_{R}^{4}}{12}-2 y_{L_{2}}^{4} \\
= & \lambda_{2 b}\left(24 \lambda_{2 a}-16 g_{L}^{2}-16 g_{R}^{2}+8 y_{L_{2}}^{2}+12 y_{Q_{2}}^{2}\right)+12 \lambda_{2 b} \lambda_{3 a}+36 \lambda_{2 b}^{2}+2 \lambda_{b} \lambda_{c} \\
& +2 \lambda_{b} \lambda_{d}+3 \lambda_{c}^{2}+3 \lambda_{d}^{2}+8 \lambda_{e} \lambda_{f}-2 g_{L}^{2} g_{R}^{2}+\frac{5 g_{L}^{4}}{4}+\frac{5 g_{R}^{4}}{4}-2 y_{L_{2}}^{4}-6 y_{Q_{2}}^{4}
\end{aligned}
$$




$$
\begin{aligned}
(4 \pi)^{2} \frac{d \lambda_{3 a}}{d \ln \mu}= & \lambda_{3 a}\left(52 \lambda_{2 a}+24 \lambda_{2 b}+6 \lambda_{a}+22 \lambda_{b}+6 \lambda_{c}+6 \lambda_{d}+48 \lambda_{e}+24 \lambda_{f}-16 g_{L}^{2}-16 g_{R}^{2}\right. \\
& \left.+2 y_{L_{1}}^{2}+6 y_{L_{2}}^{2}+3 y_{Q_{1}}^{2}+9 y_{Q_{2}}^{2}\right)+24 \lambda_{2 a} \lambda_{2 b}+12 \lambda_{2 b} \lambda_{b}+4 \lambda_{2 b} \lambda_{c}+4 \lambda_{2 b} \lambda_{d} \\
& +24 \lambda_{2 b} \lambda_{e}+8 \lambda_{2 b} \lambda_{f}+12 \lambda_{2 b}^{2}+32 \lambda_{4 b} \lambda_{a}+4 \lambda_{4 b} \lambda_{b}+8 \lambda_{4 b} \lambda_{c}+8 \lambda_{4 b} \lambda_{d}+4 \lambda_{4 b} \lambda_{e} \\
& +4 \lambda_{4 b} \lambda_{f}-4 y_{L_{1}} y_{L_{2}}^{3} \\
(4 \pi)^{2} \frac{d \lambda_{2 b}}{d \ln \mu}= & \lambda_{2 b}\left(12 \lambda_{2 a}+24 \lambda_{2 b}+6 \lambda_{a}+2 \lambda_{b}+6 \lambda_{c}+6 \lambda_{d}+8 \lambda_{e}+24 \lambda_{f}-16 g_{L}^{2}-16 g_{R}^{2}\right. \\
& \left.+2 y_{L_{1}}^{2}+6 y_{L_{2}}^{2}+3 y_{Q_{1}}^{2}+9 y_{Q_{2}}^{2}\right)+12 \lambda_{2 b} \lambda_{3 a}+4 \lambda_{3 a} \lambda_{c}+4 \lambda_{3 a} \lambda_{d}+8 \lambda_{3 a} \lambda_{f} \\
& +4 \lambda_{4 b} \lambda_{b}+8 \lambda_{4 b} \lambda_{c}+8 \lambda_{4 b} \lambda_{d}+4 \lambda_{4 b} \lambda_{e}+4 \lambda_{4 b} \lambda_{f}-4 y_{L_{1}} y_{L_{2}}^{3}-12 y_{Q_{1}} y_{Q_{2}}^{3}(\mathrm{C} .18) \\
(4 \pi)^{2} \frac{d \lambda_{4 b}}{d \ln \mu}= & \lambda_{4 b}\left(12 \lambda_{1 a}+24 \lambda_{1 b}+6 \lambda_{a}+2 \lambda_{b}+6 \lambda_{c}+6 \lambda_{d}+8 \lambda_{e}+24 \lambda_{f}-16 g_{L}^{2}-16 g_{R}^{2}\right. \\
& \left.+6 y_{L_{1}}^{2}+2 y_{L_{2}}^{2}+9 y_{Q_{1}}^{2}+3 y_{Q_{2}}^{2}\right)+12 \lambda_{1 b} \lambda_{4 b}+4 \lambda_{2 b} \lambda_{b}+6 \lambda_{2 b} \lambda_{c}+6 \lambda_{2 b} \lambda_{d} \\
& +4 \lambda_{2 b} \lambda_{e}+2 \lambda_{3 a} \lambda_{c}+2 \lambda_{3 a} \lambda_{d}+4 \lambda_{3 a} \lambda_{f}+4 \lambda_{4 b} \lambda_{c}+4 \lambda_{4 b} \lambda_{d}+8 \lambda_{4 b} \lambda_{f} \\
& -4 y_{L_{2}} y_{L_{1}}^{3}-12 y_{Q_{1}}^{3} y_{Q_{2}}
\end{aligned}
$$

$(4 \pi)^{2} \frac{d \lambda_{4 b}}{d \ln \mu}=\lambda_{4 b}\left(52 \lambda_{1 a}+24 \lambda_{1 b}+6 \lambda_{a}+22 \lambda_{b}+6 \lambda_{c}+6 \lambda_{d}+48 \lambda_{e}+24 \lambda_{f}-16 g_{L}^{2}-16 g_{R}^{2}\right.$

$$
\begin{aligned}
& \left.+6 y_{L_{1}}^{2}+2 y_{L_{2}}^{2}+9 y_{Q_{1}}^{2}+3 y_{Q_{2}}^{2}\right)+24 \lambda_{1 a} \lambda_{4 b}+12 \lambda_{1 b} \lambda_{4 b}+12 \lambda_{2 b} \lambda_{a}+2 \lambda_{2 b} \lambda_{c} \\
& +2 \lambda_{2 b} \lambda_{d}+4 \lambda_{2 b} \lambda_{f}+20 \lambda_{3 a} \lambda_{a}+4 \lambda_{3 a} \lambda_{b}+6 \lambda_{3 a} \lambda_{c}+6 \lambda_{3 a} \lambda_{d}+4 \lambda_{3 a} \lambda_{e} \\
& +12 \lambda_{4 b} \lambda_{b}+4 \lambda_{4 b} \lambda_{c}+4 \lambda_{4 b} \lambda_{d}+24 \lambda_{4 b} \lambda_{e}+8 \lambda_{4 b} \lambda_{f}-4 y_{L_{2}} y_{L_{1}}^{3}
\end{aligned}
$$

$(4 \pi)^{2} \frac{d \lambda_{a}}{d \ln \mu}=\lambda_{a}\left(40 \lambda_{1 a}+24 \lambda_{1 b}+40 \lambda_{2 a}+24 \lambda_{2 b}-16 g_{L}^{2}-16 g_{R}^{2}+4 y_{L_{1}}^{2}+4 y_{L_{2}}^{2}\right.$ $\left.+6 y_{Q_{1}}^{2}+6 y_{Q_{2}}^{2}\right)+4 \lambda_{1 a} \lambda_{b}+12 \lambda_{1 a} \lambda_{c}+12 \lambda_{1 a} \lambda_{d}+4 \lambda_{1 b} \lambda_{c}+4 \lambda_{1 b} \lambda_{d}+4 \lambda_{2 a} \lambda_{b}$$$
+12 \lambda_{2 a} \lambda_{c}+12 \lambda_{2 a} \lambda_{d}+32 \lambda_{2 b} \lambda_{4 b}+4 \lambda_{2 b} \lambda_{c}+4 \lambda_{2 b} \lambda_{d}+2 \lambda_{2 b}^{2}+68 \lambda_{3 a} \lambda_{4 b}
$$$$
+4 \lambda_{3 a}^{2}+6 \lambda_{4 b}^{2}+4 \lambda_{a}^{2}+2 \lambda_{b}^{2}+2 \lambda_{c}^{2}+2 \lambda_{d}^{2}+8 \lambda_{e}^{2}+\frac{2}{3} g_{L}^{2} g_{R}^{2}+\frac{11 g_{L}^{4}}{6}
$$$$
+\frac{11 g_{R}^{4}}{6}-4 y_{L_{1}}^{2} y_{L_{2}}^{2}
$$

$(4 \pi)^{2} \frac{d \lambda_{b}}{d \ln \mu}=2\left(2 \lambda_{1 a} \lambda_{b}+2 \lambda_{2 a} \lambda_{b}+12 \lambda_{2 b} \lambda_{3 a}+3 \lambda_{2 b}^{2}+2 \lambda_{3 a} \lambda_{4 b}+12 \lambda_{3 a}^{2}+27 \lambda_{4 b}^{2}+4 \lambda_{a} \lambda_{b}\right.$

$$
\begin{aligned}
& +6 \lambda_{b} \lambda_{c}+6 \lambda_{b} \lambda_{d}-8 \lambda_{b} g_{L}^{2}-8 \lambda_{b} g_{R}^{2}+9 \lambda_{b}^{2}+2 \lambda_{b} y_{L_{1}}^{2}+2 \lambda_{b} y_{L_{2}}^{2}+3 \lambda_{b} y_{Q_{1}}^{2} \\
& \left.+3 \lambda_{b} y_{Q_{2}}^{2}+4 \lambda_{c} \lambda_{d}+48 \lambda_{e} \lambda_{f}+44 \lambda_{e}^{2}+8 \lambda_{f}^{2}+3 g_{L}^{2} g_{R}^{2}-2 y_{L_{1}}^{2} y_{L_{2}}^{2}\right)
\end{aligned}
$$

$(4 \pi)^{2} \frac{d \lambda_{c}}{d \ln \mu}=\lambda_{c}\left(4 \lambda_{1 a}+12 \lambda_{1 b}+4 \lambda_{2 a}+12 \lambda_{2 b}+8 \lambda_{a}-16 g_{L}^{2}-16 g_{R}^{2}+4 y_{L_{1}}^{2}+4 y_{L_{2}}^{2}\right.$ 


$$
\begin{aligned}
&\left.+6 y_{Q_{1}}^{2}+6 y_{Q_{2}}^{2}\right)+4 \lambda_{1 b} \lambda_{b}+8 \lambda_{2 b} \lambda_{3 a}+16 \lambda_{2 b} \lambda_{4 b}+4 \lambda_{2 b} \lambda_{b}+6 \lambda_{2 b}^{2} \\
&+4 \lambda_{3 a} \lambda_{4 b}+14 \lambda_{4 b}^{2}+4 \lambda_{b} \lambda_{d}+6 \lambda_{c}^{2}+16 \lambda_{e} \lambda_{f}+24 \lambda_{f}^{2}-2 g_{L}^{2} g_{R}^{2}+\frac{5 g_{R}^{4}}{2} \\
&-4 y_{L_{1}}^{2} y_{L_{2}}^{2}-12 y_{Q_{1}}^{2} y_{Q_{2}}^{2} \\
&(4 \pi)^{2} \frac{d \lambda_{d}}{d \ln \mu}= \lambda_{d}\left(4 \lambda_{1 a}+12 \lambda_{1 b}+4 \lambda_{2 a}+12 \lambda_{2 b}+8 \lambda_{a}-16 g_{L}^{2}-16 g_{R}^{2}+4 y_{L_{1}}^{2}+4 y_{L_{2}}^{2}\right. \\
&\left.+6 y_{Q_{1}}^{2}+6 y_{Q_{2}}^{2}\right)+4 \lambda_{1 b} \lambda_{b}+8 \lambda_{2 b} \lambda_{3 a}+16 \lambda_{2 b} \lambda_{4 b}+4 \lambda_{2 b} \lambda_{b}+6 \lambda_{2 b}^{2}+4 \lambda_{3 a} \lambda_{4 b} \\
&+14 \lambda_{4 b}^{2}+4 \lambda_{b} \lambda_{c}+6 \lambda_{d}^{2}+16 \lambda_{e} \lambda_{f}+24 \lambda_{f}^{2}-2 g_{L}^{2} g_{R}^{2}+\frac{5 g_{L}^{4}}{2} \\
&-4 y_{L_{1}}^{2} y_{L_{2}}^{2}-12 y_{Q_{1}}^{2} y_{Q_{2}}^{2} \\
&(4 \pi)^{2} \frac{d \lambda_{e}}{d \ln \mu}= 4 \lambda_{1 a} \lambda_{e}+4 \lambda_{1 b} \lambda_{f}+4 \lambda_{2 a} \lambda_{e}+12 \lambda_{2 b} \lambda_{3 a}+2 \lambda_{2 b} \lambda_{4 b}+4 \lambda_{2 b} \lambda_{f}+2 \lambda_{2 b}^{2}+2 \lambda_{3 a} \lambda_{4 b} \\
&+12 \lambda_{3 a}^{2}+26 \lambda_{4 b}^{2}+8 \lambda_{a} \lambda_{e}+40 \lambda_{b} \lambda_{e}+24 \lambda_{b} \lambda_{f}+12 \lambda_{c} \lambda_{e}+4 \lambda_{c} \lambda_{f}+12 \lambda_{d} \lambda_{e} \\
&+4 \lambda_{d} \lambda_{f}-16 \lambda_{e} g_{L}^{2}-16 \lambda_{e} g_{R}^{2}+4 \lambda_{e} y_{L_{1}}^{2}+4 \lambda_{e} y_{L_{2}}^{2}+6 \lambda_{e} y_{Q_{1}}^{2} \\
&+6 \lambda_{e} y_{Q_{2}}^{2}-2 y_{L_{1}}^{2} y_{L_{2}}^{2} \\
&= \lambda_{f}\left(4 \lambda_{1 a}+4 \lambda_{2 a}+8 \lambda_{a}+12 \lambda_{c}+12 \lambda_{d}-16 g_{L}^{2}-16 g_{R}^{2}+4 y_{L_{1}}^{2}+4 y_{L_{2}}^{2}\right. \\
&\left.+6 y_{Q_{1}}^{2}+6 y_{Q_{2}}^{2}\right)+4 \lambda_{1 b} \lambda_{e}+4 \lambda_{2 b} \lambda_{3 a}+2 \lambda_{2 b} \lambda_{4 b}+4 \lambda_{2 b} \lambda_{e}+6 \lambda_{2 b}^{2}+2 \lambda_{3 a} \lambda_{4 b} \\
&+10 \lambda_{4 b}^{2}+4 \lambda_{c} \lambda_{e}+4 \lambda_{d} \lambda_{e}-2 y_{L_{1}}^{2} y_{L_{2}}^{2}-6 y_{Q_{1}}^{2} y_{Q_{2}}^{2} \\
&(4 \pi)^{2} \frac{d \lambda_{f}}{d \ln \mu}
\end{aligned}
$$

Open Access. This article is distributed under the terms of the Creative Commons Attribution License (CC-BY 4.0), which permits any use, distribution and reproduction in any medium, provided the original author(s) and source are credited.

\section{References}

[1] M. Farina, D. Pappadopulo and A. Strumia, A modified naturalness principle and its experimental tests, JHEP 08 (2013) 022 [arXiv: 1303.7244] [INSPIRE].

[2] J.P. Fatelo, J.M. Gerard, T. Hambye and J. Weyers, Symmetry breaking induced by top loops, Phys. Rev. Lett. 74 (1995) 492 [INSPIRE].

[3] T. Hambye, Symmetry breaking induced by top quark loops from a model without scalar mass, Phys. Lett. B 371 (1996) 87 [hep-ph/9510266] [INSPIRE].

[4] R. Hempfling, The next-to-minimal Coleman-Weinberg model, Phys. Lett. B 379 (1996) 153 [hep-ph/9604278] [INSPIRE].

[5] F. Vissani, Do experiments suggest a hierarchy problem?, Phys. Rev. D 57 (1998) 7027 [hep-ph/9709409] [INSPIRE].

[6] P.H. Frampton and C. Vafa, Conformal approach to particle phenomenology, hep-th/9903226 [INSPIRE]. 
[7] K.A. Meissner and H. Nicolai, Conformal symmetry and the standard model, Phys. Lett. B 648 (2007) 312 [hep-th/0612165] [INSPIRE].

[8] W.-F. Chang, J.N. Ng and J.M.S. Wu, Shadow Higgs from a scale-invariant hidden U(1)(s) model, Phys. Rev. D 75 (2007) 115016 [hep-ph/0701254] [InSPIRE].

[9] R. Foot, A. Kobakhidze and R.R. Volkas, Electroweak Higgs as a pseudo-Goldstone boson of broken scale invariance, Phys. Lett. B 655 (2007) 156 [arXiv:0704.1165] [InSPIRE].

[10] R. Foot, A. Kobakhidze, K.L. McDonald and R.R. Volkas, A solution to the hierarchy problem from an almost decoupled hidden sector within a classically scale invariant theory, Phys. Rev. D 77 (2008) 035006 [arXiv:0709.2750] [INSPIRE].

[11] S. Iso, N. Okada and Y. Orikasa, The minimal B-L model naturally realized at TeV scale, Phys. Rev. D 80 (2009) 115007 [arXiv:0909.0128] [inSPIRE].

[12] M. Shaposhnikov and C. Wetterich, Asymptotic safety of gravity and the Higgs boson mass, Phys. Lett. B 683 (2010) 196 [arXiv:0912.0208] [INSPIRE].

[13] S. Iso and Y. Orikasa, TeV scale B-L model with a flat Higgs potential at the Planck scale in view of the hierarchy problem, PTEP 2013 (2013) 023B08 [arXiv:1210.2848] [INSPIRE].

[14] T. Hur and P. Ko, Scale invariant extension of the standard model with strongly interacting hidden sector, Phys. Rev. Lett. 106 (2011) 141802 [arXiv:1103.2571] [INSPIRE].

[15] M. Shaposhnikov, Asymptotic safety of gravity and the Higgs-boson mass, Theor. Math. Phys. 170 (2012) 229 [Teor. Mat. Fiz. 170 (2012) 280] [INSPIRE].

[16] C. Englert, J. Jaeckel, V.V. Khoze and M. Spannowsky, Emergence of the electroweak scale through the Higgs portal, JHEP 04 (2013) 060 [arXiv:1301.4224] [INSPIRE].

[17] E.J. Chun, S. Jung and H.M. Lee, Radiative generation of the Higgs potential, Phys. Lett. B 725 (2013) 158 [arXiv:1304.5815] [INSPIRE].

[18] M. Heikinheimo, A. Racioppi, M. Raidal, C. Spethmann and K. Tuominen, Physical Naturalness and Dynamical Breaking of Classical Scale Invariance, Mod. Phys. Lett. A 29 (2014) 1450077 [arXiv: 1304.7006] [INSPIRE].

[19] T. Hambye and A. Strumia, Dynamical generation of the weak and Dark Matter scale, Phys. Rev. D 88 (2013) 055022 [arXiv: 1306.2329] [INSPIRE].

[20] C.D. Carone and R. Ramos, Classical scale-invariance, the electroweak scale and vector dark matter, Phys. Rev. D 88 (2013) 055020 [arXiv:1307.8428] [INSPIRE].

[21] A. Farzinnia, H.-J. He and J. Ren, Natural electroweak symmetry breaking from scale invariant Higgs mechanism, Phys. Lett. B 727 (2013) 141 [arXiv:1308.0295] [INSPIRE].

[22] R. Foot, A. Kobakhidze, K.L. McDonald and R.R. Volkas, Poincaré protection for a natural electroweak scale, Phys. Rev. D 89 (2014) 115018 [arXiv:1310.0223] [INSPIRE].

[23] D. Chway, T.H. Jung, H.D. Kim and R. Dermisek, Radiative electroweak symmetry breaking model perturbative all the way to the Planck scale, Phys. Rev. Lett. 113 (2014) 051801 [arXiv: 1308.0891] [INSPIRE].

[24] M. Holthausen, J. Kubo, K.S. Lim and M. Lindner, Electroweak and conformal symmetry breaking by a strongly coupled hidden sector, JHEP 12 (2013) 076 [arXiv:1310.4423] [INSPIRE].

[25] C.T. Hill, Is the Higgs boson associated with Coleman-Weinberg dynamical symmetry breaking?, Phys. Rev. D 89 (2014) 073003 [arXiv:1401.4185] [INSPIRE]. 
[26] J. Guo and Z. Kang, Higgs naturalness and dark matter stability by scale invariance, arXiv: 1401.5609 [INSPIRE].

[27] S. Benic and B. Radovcic, Electroweak breaking and Dark Matter from the common scale, Phys. Lett. B 732 (2014) 91 [arXiv:1401.8183] [inSPIRE].

[28] P.H. Chankowski, A. Lewandowski, K.A. Meissner and H. Nicolai, Softly broken conformal symmetry and the stability of the electroweak scale, Mod. Phys. Lett. A 30 (2015) 1550006 [arXiv: 1404.0548] [INSPIRE].

[29] H. Davoudiasl and I.M. Lewis, Right-handed neutrinos as the origin of the electroweak scale, Phys. Rev. D 90 (2014) 033003 [arXiv:1404.6260] [inSPIRE].

[30] K. Allison, C.T. Hill and G.G. Ross, Ultra-weak sector, Higgs boson mass and the dilaton, Phys. Lett. B 738 (2014) 191 [arXiv: 1404.6268] [INSPIRE].

[31] G.M. Pelaggi, Predictions of a model of weak scale from dynamical breaking of scale invariance, arXiv:1406.4104 [INSPIRE].

[32] W. Altmannshofer, W.A. Bardeen, M. Bauer, M. Carena and J.D. Lykken, Light dark matter, naturalness and the radiative origin of the electroweak scale, JHEP 01 (2015) 032 [arXiv: 1408.3429] [INSPIRE].

[33] R. Foot, A. Kobakhidze and A. Spencer-Smith, Criticality in the scale invariant standard model (squared), arXiv: 1409.4915 [INSPIRE].

[34] M.B. Einhorn and D.R.T. Jones, Naturalness and dimensional transmutation in classically scale-invariant gravity, arXiv:1410.8513 [INSPIRE].

[35] O. Antipin, M. Redi and A. Strumia, Dynamical generation of the weak and dark matter scales from strong interactions, JHEP 01 (2015) 157 [arXiv:1410.1817] [INSPIRE].

[36] I. Antoniadis, A possible new dimension at a few TeV, Phys. Lett. B 246 (1990) 377 [INSPIRE].

[37] R. Sundrum, Fat euclidean gravity with small cosmological constant, Nucl. Phys. B 690 (2004) 302 [hep-th/0310251] [INSPIRE].

[38] A. Salvio and A. Strumia, Agravity, JHEP 06 (2014) 080 [arXiv:1403.4226] [INSPIRE].

[39] D.F. Litim and F. Sannino, Asymptotic safety guaranteed, JHEP 12 (2014) 178 [arXiv:1406.2337] [INSPIRE].

[40] P.J. Redmond and J.L. Uretsky, Conjecture concerning the properties of nonrenormalizable field theories, Phys. Rev. Lett. 1 (1958) 147 [INSPIRE].

[41] N.N. Bogolyubov, A.A. Logunov and D.V. Shirkov, The method of dispersion relations and perturbation theory Sov. Phys. JETP 37 (1960) 574.

[42] I.M. Suslov, Renormalization group functions of the $\phi^{4}$ theory in the strong coupling limit: analytical results, J. Exp. Theor. Phys. 107 (2008) 413 [Zh. Eksp. Teor. Fiz. 134 (2008) 490] [arXiv: 1010.4081] [INSPIRE].

[43] I.M. Suslov, Exact asymptotics for $\beta$-function in QED, J. Exp. Theor. Phys. 108 (2009) 980 [arXiv:0804.2650] [INSPIRE].

[44] N. Seiberg and E. Witten, Electric-magnetic duality, monopole condensation and confinement in $N=2$ supersymmetric Yang-Mills theory, Nucl. Phys. B 426 (1994) 19 [Erratum ibid. B 430 (1994) 485] [hep-th/9407087] [INSPIRE]. 
[45] D.F. Litim, M. Mojaza and F. Sannino, Vacuum stability of asymptotically safe gauge-Yukawa theories, arXiv:1501.03061 [INSPIRE].

[46] G. Marques Tavares, M. Schmaltz and W. Skiba, Higgs mass naturalness and scale invariance in the UV, Phys. Rev. D 89 (2014) 015009 [arXiv:1308.0025] [INSPIRE].

[47] D.J. Gross and F. Wilczek, Asymptotically free gauge theories. 1, Phys. Rev. D 8 (1973) 3633 [INSPIRE].

[48] D.J. Gross and F. Wilczek, Ultraviolet behavior of nonabelian gauge theories, Phys. Rev. Lett. 30 (1973) 1343 [INSPIRE].

[49] D.J. Gross and F. Wilczek, Asymptotically free gauge theories. 2, Phys. Rev. D 9 (1974) 980 [INSPIRE].

[50] H.D. Politzer, Reliable perturbative results for strong interactions?, Phys. Rev. Lett. 30 (1973) 1346 [INSPIRE].

[51] S.R. Coleman and D.J. Gross, Price of asymptotic freedom, Phys. Rev. Lett. 31 (1973) 851 [INSPIRE].

[52] T.P. Cheng, E. Eichten and L.-F. Li, Higgs phenomena in asymptotically free gauge theories, Phys. Rev. D 9 (1974) 2259 [inSPIRE].

[53] E.S. Fradkin and O.K. Kalashnikov, Asymptotically free SU(5) model of unified interaction, Phys. Lett. B 64 (1976) 177 [INSPIRE].

[54] O.K. Kalashnikov, Asymptotically free models of unified interaction, Lebedev-77-206 (1977) [INSPIRE].

[55] E.S. Fradkin, O.K. Kalashnikov and S.E. Konshtein, Asymptotically free $E_{6}$ model of unified interaction, Lett. Nuovo Cim. 21 (1978) 5 [INSPIRE].

[56] O.K. Kalashnikov, Asymptotically free $\mathrm{SU}(2) \times \mathrm{SU}(2) \times \mathrm{SU}(4)$ model of unified interaction, Phys. Lett. B 72 (1977) 65 [inSPIRE].

[57] B. Pendleton and G.G. Ross, Mass and mixing angle predictions from infrared fixed points, Phys. Lett. B 98 (1981) 291 [INSPIRE].

[58] S.R. Coleman and E.J. Weinberg, Radiative corrections as the origin of spontaneous symmetry breaking, Phys. Rev. D 7 (1973) 1888 [INSPIRE].

[59] C.G. Callan and S. Coleman, The fate of the false vacuum. 2. First quantum corrections, Phys. Rev. D 16 (1977) 1762 [INSPIRE].

[60] G. Isidori, G. Ridolfi and A. Strumia, On the metastability of the standard model vacuum, Nucl. Phys. B 609 (2001) 387 [hep-ph/0104016] [INSPIRE].

[61] J.R. Espinosa, G.F. Giudice and A. Riotto, Cosmological implications of the Higgs mass measurement, JCAP 05 (2008) 002 [arXiv:0710.2484] [INSPIRE].

[62] J. Elias-Miro et al., Higgs mass implications on the stability of the electroweak vacuum, Phys. Lett. B 709 (2012) 222 [arXiv:1112.3022] [INSPIRE].

[63] G. Degrassi et al., Higgs mass and vacuum stability in the standard model at NNLO, JHEP 08 (2012) 098 [arXiv: 1205.6497] [INSPIRE].

[64] D. Buttazzo et al., Investigating the near-criticality of the Higgs boson, JHEP 12 (2013) 089 [arXiv: 1307.3536] [INSPIRE]. 
[65] S.C. Frautschi and J. Kim, SU(5) Higgs problem with adjoint + vector representations, Nucl. Phys. B 196 (1982) 301 [INSPIRE].

[66] K.G. Klimenko, On necessary and sufficient conditions for some Higgs potentials to be bounded from below, Theor. Math. Phys. 62 (1985) 58 [InSPIRE].

[67] K. Kannike, Vacuum stability conditions from copositivity criteria, Eur. Phys. J. C 72 (2012) 2093 [arXiv: 1205.3781] [INSPIRE].

[68] G. D'Ambrosio, G.F. Giudice, G. Isidori and A. Strumia, Minimal flavor violation: an effective field theory approach, Nucl. Phys. B 645 (2002) 155 [hep-ph/0207036] [INSPIRE].

[69] A.J. Buras, K. Gemmler and G. Isidori, Quark flavour mixing with right-handed currents: an effective theory approach, Nucl. Phys. B 843 (2011) 107 [arXiv: 1007.1993] [INSPIRE].

[70] G. Isidori, Flavor physics and CP-violation, arXiv:1302.0661 [INSPIRE].

[71] CMS collaboration, Search for heavy narrow dilepton resonances in pp collisions at $\sqrt{s}=7$ $\mathrm{TeV}$ and $\sqrt{s}=8 \mathrm{TeV}$, Phys. Lett. B 720 (2013) 63 [arXiv:1212.6175] [INSPIRE].

[72] CMS collaboration, Search for narrow resonances using the dijet mass spectrum in pp collisions at $\sqrt{s}=8 \mathrm{TeV}$, Phys. Rev. D 87 (2013) 114015 [arXiv:1302.4794] [INSPIRE].

[73] ATLAS collaboration, Search for high-mass dilepton resonances in pp collisions at $\sqrt{s}=8 \mathrm{TeV}$ with the ATLAS detector, Phys. Rev. D 90 (2014) 052005 [arXiv:1405.4123] [INSPIRE].

[74] ATLAS collaboration, Search for new phenomena in the dijet mass distribution using pp collision data at $\sqrt{s}=8 \mathrm{TeV}$ with the ATLAS detector, arXiv:1407.1376 [INSPIRE].

[75] E. Salvioni, G. Villadoro and F. Zwirner, Minimal $Z^{\prime}$ models: present bounds and early LHC reach, JHEP 11 (2009) 068 [arXiv:0909.1320] [INSPIRE].

[76] E. Salvioni, A. Strumia, G. Villadoro and F. Zwirner, Non-universal minimal $Z^{\prime}$ models: present bounds and early LHC reach, JHEP 03 (2010) 010 [arXiv:0911.1450] [INSPIRE].

[77] R. Barbieri, A. Pomarol, R. Rattazzi and A. Strumia, Electroweak symmetry breaking after LEP-1 and LEP-2, Nucl. Phys. B 703 (2004) 127 [hep-ph/0405040] [INSPIRE].

[78] G. Cacciapaglia, C. Csáki, G. Marandella and A. Strumia, The minimal set of electroweak precision parameters, Phys. Rev. D 74 (2006) 033011 [hep-ph/0604111] [INSPIRE].

[79] CMS collaboration, Search for heavy neutrinos and $\mathrm{W}$ bosons with right-handed couplings in proton-proton collisions at $\sqrt{s}=8 \mathrm{TeV}$, Eur. Phys. J. C 74 (2014) 3149 [arXiv:1407.3683] [INSPIRE].

[80] V. Cirigliano, M. Gonzalez-Alonso and M.L. Graesser, Non-standard charged current interactions: $\beta$ decays versus the LHC, JHEP 02 (2013) 046 [arXiv:1210.4553] [INSPIRE].

[81] R. Foot, An alternative $\mathrm{SU}(4) \times \mathrm{SU}(2)_{L} \times \mathrm{SU}(2)_{R}$ model, Phys. Lett. B 420 (1998) 333 [hep-ph/9708205] [INSPIRE].

[82] R.R. Volkas, Prospects for mass unification at low-energy scales, Phys. Rev. D 53 (1996) 2681 [hep-ph/9507215] [INSPIRE].

[83] G. Valencia and S. Willenbrock, Quark-lepton unification and rare meson decays, Phys. Rev. D 50 (1994) 6843 [hep-ph/9409201] [INSPIRE].

[84] M. Carpentier and S. Davidson, Constraints on two-lepton, two quark operators, Eur. Phys. J. C 70 (2010) 1071 [arXiv:1008.0280] [INSPIRE]. 
[85] QCDSF collaboration, G.S. Bali et al., The strange and light quark contributions to the nucleon mass from Lattice QCD, Phys. Rev. D 85 (2012) 054502 [arXiv:1111.1600] [INSPIRE]. 